\title{
Analysis of the Relationship between the Density and Lane-Changing Behavior of Circular Multilane Urban Expressway in Mixed Traffic
}

\author{
Han Xie ${ }^{\mathbb{D}}$, Juanxiu Zhu $(\mathbb{D}$, and Huawei Duan \\ Xihua University, School of Management, Jinniu Disitrict Jinzhou Road 999, Chengdu, China \\ Correspondence should be addressed to Juanxiu Zhu; zhujuanxiu@163.com
}

Received 14 October 2021; Accepted 15 November 2021; Published 4 January 2022

Academic Editor: Gen Li

Copyright $\odot 2022$ Han Xie et al. This is an open access article distributed under the Creative Commons Attribution License, which permits unrestricted use, distribution, and reproduction in any medium, provided the original work is properly cited.

\begin{abstract}
The behavior of changing lanes has a great impact on road traffic with heavy traffic. Traffic flow density is one of the important parameters that characterize the characteristics of traffic flow, and it will also be affected by the behavior of changing lanes, especially in the case of each lane. The penetration of autonomous vehicles can effectively reduce lane-changing behavior. Studying the relationship between traffic flow density and lane-changing behavior under different autonomous vehicle penetration rates is of great significance for describing the operation mechanism of mixed traffic flow and the control of mixed traffic. In this article, we use empirical, simulation, and data-driven methods to analyze the urban expressway of autonomous vehicles with penetration rates of $10 \%, 20 \%, 30 \%, 40 \%, 50 \%, 60 \%, 70 \%$, and $80 \%$, respectively. A simulation experiment was carried out on the road, and data related to density, the rate of changing into the lanes, and the rate of changing out lanes were collected. The analysis of the experimental results found the following: (1) The increase in penetration of autonomous vehicles leads to a certain degree of downward trend in density, the rate of changing into the lanes, and the rate of changing out lanes. (2) Different lanes have different effects on the penetration of autonomous vehicles. In a 4-lane road, the two lanes farther from the entrance and exit are closer in appearance, while the two lanes closer to the entrance and exit are similar. (3) The relationship between density and the rate of changing into the lanes and the rate of changing out lanes shows a linear relationship with the penetration of autonomous vehicles. Although the performance of each lane is slightly different, in general, it can be carried out by a multiple regression model. The given parameter value range is relatively close under different permeability. In summary, autonomous vehicles effectively reduce the traffic density and lane-changing behavior of each lane. There is a linear relationship between traffic flow density and lane-changing behavior with the penetration of autonomous vehicles. The density-lane-changing behavior model proposed in this paper can better describe the relationship between the density of the circular multilane urban expressway and the lane-changing behavior in the case of a large traffic flow in mixed traffic.
\end{abstract}

\section{Introduction}

The relationship between traffic flow density and lanechanging behavior is very important for understanding the mechanism of traffic flow. Traffic flow density is one of the important parameters of traffic flow, which can directly reflect traffic demand and is also the basis for the classification of road service levels. The behavior of changing lanes can quickly change the density of traffic flow, especially on a single lane. Therefore, studying the relationship between traffic flow density and lane-changing behavior is an important part of understanding the operation mechanism of urban expressway traffic flow.

With the development of artificial intelligence, autonomous vehicles have gradually appeared in people's sight in recent years. Vehicles in the controlled environment will adopt car-following behavior in most cases and will adopt lane-changing behaviors when there are obstacles in front or need to change lanes to reach the target lane. Compared with humans, the lane-changing behavior of autonomous vehicle is more concerned with safety and standardization. When autonomous vehicles are driving on roads in mixed traffic, 
they will affect the road traffic flow [1-4]. At present, there are many studies on lane-changing behavior of a single autonomous vehicle [5-8], and most of the research studies used data-driven and simulation methods. There are fewer studies on the influence of lane-changing behavior on density and fewer studies on the relationship between individual lanes. As an important traffic flow parameter, it is important to understand the impact of autonomous vehicles on traffic flow density and lane-changing behavior in mixed traffic.

This paper uses empirical, simulation, and data-driven methods to study the relationship between density and lanechanging behavior under mixed traffic conditions. Taking a typical ring-shaped multilane urban expressway [9], the third ring expressway in Chengdu, China, as the simulation model benchmark object, this study first adopts empirical research, through the traffic flow density, the rate of changing into lanes, and the rate of changing out lanes of the third ring expressway, and data collection and analysis were carried out to verify the validity of the data. Then, the method of benchmarking the three-loop modeling was adopted to establish the corresponding simulation model and compare the empirical data to verify the usability of the simulation model. Finally, 8 simulation experiments with the penetration rate of $10 \%, 20 \%, 30 \%, 40 \%, 50 \%, 60 \%, 70 \%$, and $80 \%$ of autonomous vehicles are designed to collect the data of traffic flow density, the rate of changing into lanes, and the rate of changing out lanes. A multiple linear regression model of density and lane-changing behavior was proposed for the first time through a data-driven method. And the parameter value range was given. The model better describes the relationship between the density and the lanechanging behavior of a typical circular multilane urban expressway in a mixed traffic environment when the traffic volume is large.

The first part of this article is an introduction to the research background. The second part is the method used in the article, specifically for empirical research and simulation model establishment. The third part introduces simulation experiments and experimental results. The fourth part analyzes the experimental results and proposes the established relationship model between density and lane-changing behavior. Finally, a summary is made.

1.1. Literature Review. Traffic flow density is an important parameter that characterizes the state of traffic flow. At present, most of the research on density is based on basic graph theory. The basic graph is the basic theory of traffic flow. The theory describes the nonlinear relationship between traffic flow, speed, and density, and a pairwise relationship model of three parameters is given. The basic graph theory is based on a human driving environment. At present, scholars have proposed the basic graph of mixed traffic flow. The research mainly adopts two kinds of simulation research and theoretical research. In simulation research, the current focus is on the flow-density relationship [10-14] in the non-full-density state. In terms of theoretical analysis, the average headway distance is often used in the research studies, and the relationship between flow-speed-density is derived [14-18]. The above research studies are based on car-following behavior. As the main behavior of autonomous vehicles running on road, in addition to car-following, there are also lane-changing behaviors. There are few studies on the impact of lanechanging behavior on traffic flow in mixed traffic. The relationship between traffic flow density and lane-changing behavior under mixed traffic conditions has not been studied.

At present, the research studies on the impact of lanechanging behavior on traffic flow are still focused on the traffic flow model. In the human driving, lane-changing behavior is basically based on the LWR model. The lanechanging behavior is used as parameters [19-21] or factors [22]. In HCM2010 [23], the lane-changing rate is introduced for the interweaving lanes of expressways, but the relationship between lane-changing behavior and density is not analyzed. In the mixed traffic, there are few studies on the influence of lane-changing behavior on traffic flow, and more researches focus on the study of lane-changing behavior of autonomous vehicles. The research studies on lanechanging behavior of autonomous vehicles mainly include lane-changing intention $[24,25]$, lane-changing decision $[26,27]$, cooperative lane-changing between front and rear vehicles [28-31], and lane-changing position [32]. Research studies use human behavior as the research basis to construct human-like behavior models. Although there is a gap with human behavior, compared with human behavior, selfdriving vehicles can reach a partial or full information state when making decisions, and the accuracy of decisionmaking has also been improved. However, the above research is only conducted from autonomous vehicles, and the research on the impact of lane-changing behavior on traffic flow in mixed traffic is not involved. With the further development of artificial intelligence, it can be predicted that, in the future urban traffic, there will be a state where autonomous vehicles and human-driven vehicles will be mixed. It is very important to understand the operation mechanism of traffic flow in the mixed state. Studying the relationship between density, which is one of the important parameters of traffic flow, and lane-changing behavior, which is one of the main behaviors of vehicles in traffic, is of great significance for understanding the operation mechanism of traffic flow in mixed traffic.

In summary, there are few studies on the influence of lane-changing behavior on density in mixed traffic. Lanechanging behavior is only used as a factor or parameter to participate in the study of traffic flow in the human driving situation. And the relationship between lane-changing behavior and traffic flow parameters has not been studied. The behavior of changing lanes has the greatest impact on each lane on the road, especially in heavy traffic. The urban expressway fits this situation. In the case of frequent entrances and exits, more lane-changing behaviors, greater traffic flow, and more interweaving areas, the relationship between lanechanging behavior and density under non-full traffic flow conditions can be better studied. Therefore, it is feasible to use a typical circular multilane urban expressway as the 
benchmark object for empirical research and simulation modeling to conduct simulation experiments to study the relationship between traffic flow density and lane-changing behavior in a mixed state. A clear relationship between density and lane-changing behavior in mixed traffic is of great significance for understanding the traffic flow mechanism of typical circular multilane urban expressways in mixed traffic, and it can also provide a theoretical basis for future mixed traffic management and control.

\section{Research Protocols}

Empirical, simulation, and data-driven methods are used in this paper to verify the effectiveness and accuracy of the empirical data. Furthermore, a simulation model is established, corresponding data through simulation experiments are collected, and a data-driven method is used to establish a model of the relationship between density and lanechanging behavior. In this part, empirical data testing and simulation modeling methods are used.

2.1. Empirical Research and Data Testing. In this paper, the data used in this study is to extract data from videos, and the video data were collected from 2011, 2015, and 2018. The videos come from on-site shooting and Chengdu Transportation Administration. The data is extracted from the videos using manual collection and self-developed traffic flow data collection software. The distribution of the collection points is shown in Figure 1.

In Figure 1, there are a total of 9 collection points. The collection points consider the surrounding land use, the connection mode of the road section and the overpass, and whether it is convenient for data collection and other conditions. Through on-site inspections, map selection, and inspection of relevant specifications, the final selection is consistent with connecting entrances and exits, pedestrian bridges, and roads for commercial and residential use, and the connection with the overpass is connecting straight and connecting with the overpass and connecting the curved road. Data collection is performed on 9 road sections connected with an overpass, connected to a straight road but not connected to an overpass, and connected to a curve but not connected to an overpass in one of four ways. In this study, data of traffic flow density, the rate of changing into lanes, and the rate of changing out lanes are collected. The collection is carried out in the morning and afternoon of the working day with heavy traffic.

In order to make the data representative of roads, the minimum sample size is adopted as formula (1) [33], and the minimum sample size is calculated to be 48 . That is, as long as the data is larger than 48 groups, it can represent the road characteristics.

$$
n \geq\left(\frac{\sigma \bullet K}{E}\right)^{2},
$$

where $n$ is the minimum sample size for observation; $\sigma$ is the standard deviation of the sample size of the observed vehicle speed; $K$ is the constant level of confidence to meet

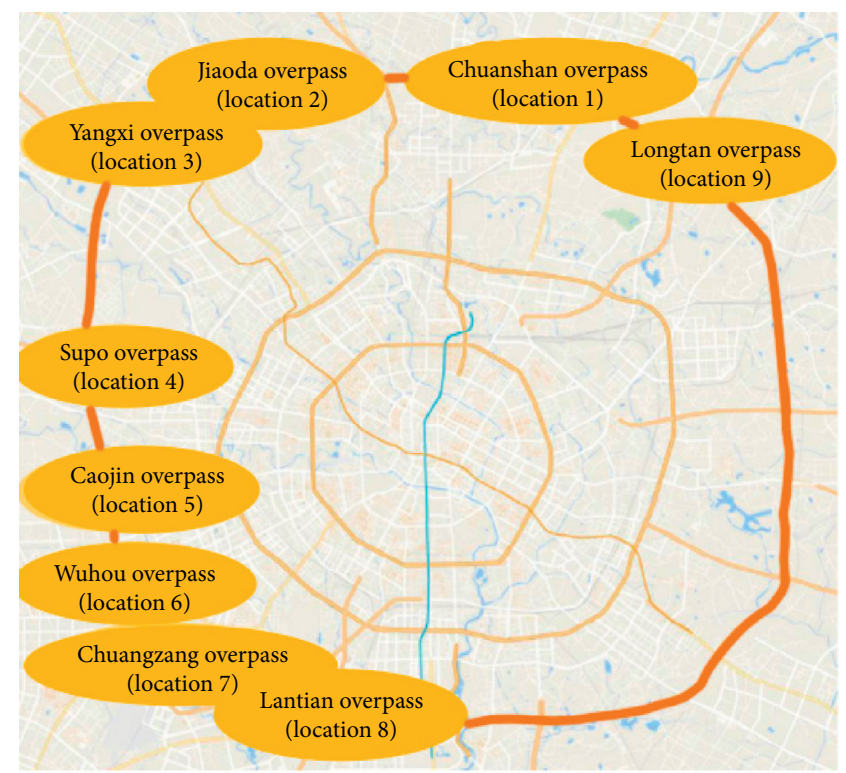

Figure 1: Collection point situation.

expectations; and $E$ is the allowable error of vehicle speed depending on the accuracy of the average vehicle speed, generally $1.5 \sim 2 \mathrm{~km} / \mathrm{h}$.

In the formula, the parameter value is $\sigma=7 \mathrm{~km} / \mathrm{h}$ (according to two-way eight lanes), $K=1.96$ (95\%), and $E=2 \mathrm{~km} / \mathrm{h}$, and after calculation, the minimum sample size is 48 .

The collected data at each point is compared with the video data to eliminate the collection of unreasonable data. Finally, the number of available data groups is at least 55 groups and at most 179 groups.

The density, the rate of changing into lanes, and the rate of changing out lanes studied in this paper are defined as follows.

The density $k$ refers to the number of vehicles present on the road per unit length at a certain moment [33], as shown in the following formula:

$$
k=\frac{N}{L},
$$

where $k$ is the traffic flow density at a certain moment (vehicles $/ \mathrm{km}$ ), $N$ is the number of vehicles, and $L$ is the length of road section.

The photography method used in the density collection method [34] is shown as follows:

$$
k=\frac{\sum_{i=0}^{n} k_{i}}{n} \times \frac{1}{L}
$$

where $n$ is the total time, $i$ is the number of screens when reading the number of vehicles, $k_{i}$ is the number of vehicles in the interval measured on the $i$-th screen, and $L$ is the length of observation interval $(\mathrm{km})$.

The rate of changing into lanes and the rate of changing out lanes are used to describe the behavior of a vehicle driving on the road changing into the intended lane and changing out of the current lane, as shown in Figure 2. 


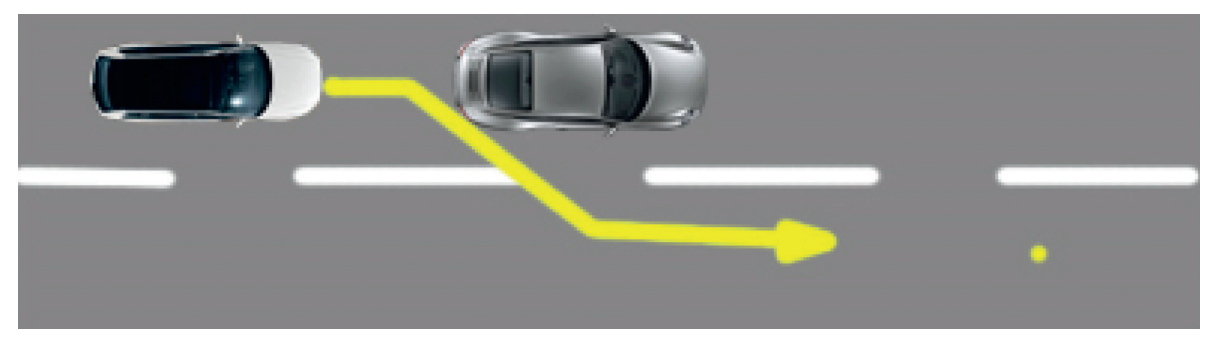

FIGURE 2: Schematic diagram of vehicle changing lanes (picture from the Internet).

In Figure 2, the white vehicle is a vehicle that intends to change lanes. For some reason, the white vehicle is not satisfied with the current driving lane, so it intends to change lanes from the current driving lane and continue driving. When the vehicle changes lanes, there are two states: partially entering the lane and fully entering the lane. This paper uses more than $50 \%$ of the vehicle's location to determine whether the vehicle belongs to the state of changing into the lane or changing out of the lane, so as to include the rate of changing into lanes and the rate of changing out lanes.

The rate of changing into lanes is the number of vehicles changing into the lane, which refers to the number of vehicles entering the tested lane from other lanes within a unit time and unit length. Similarly, the rate of changing out lanes is also the number of vehicles that change lanes, that is, the number of vehicles entering other lanes from the tested lane in a unit time and unit length. Here the unit time is $1 \mathrm{~h}$, and the unit length is $1 \mathrm{~km}$. The definition of the rate of changing into lanes and the rate of changing out lanes is consistent with HCM2010 [23]. However, HCM2010 [23] did not conduct research on the sections of the vehicle on and off the ramp. The main reason is that the main research object of HCM2010 [23] is the expressway. The distance between the on-ramps on the expressway is relatively long, and the ramp has a short influence on the road section, while the distance between the on-ramps on the urban expressway is almost in the interlaced area. The change of lanes of incoming and outgoing vehicles has a greater impact. If only the vehicles on and off the ramp are considered, and the lane-changing behavior in the road section is not considered, the impact of lane-changing behavior on expressways cannot be better described.

In the empirical research, the data collection time interval is $5 \mathrm{~min}$, and the distance length is $100 \mathrm{~m}$ or $200 \mathrm{~m}$. Before conducting the research, the data was standardized and converted, and the final data time interval was $1 \mathrm{~h}$, and the distance length was $1 \mathrm{~km}$. The unit of the rate of changing into lanes and the rate of changing out lanes is the number of lane changes in HCM2010 [23], which are represented by LCi and LCo, respectively.

Due to the discontinuity of the collection location and time, some of the collected data are missing. The time difference method formula (4) [33] was used to complete the data. A total of 923 data were obtained after completion. The data distribution box diagram of the density, the rate of changing into lanes, and the rate of changing out lanes of a single lane is shown in Figure 3.

$$
Z^{t}\left(t_{j}, x_{i}\right)= \begin{cases}z\left(t_{a}, x_{i}\right), & t_{j}=t_{1} \\ z\left(t_{j-1}, x_{i}\right)+\frac{\Delta t}{t_{j-1}-t_{a}} z\left(t_{a}, x_{i}\right), & t_{1}<t_{j}<t_{p}, \\ z\left(t_{j}, x_{i-1}\right) & t_{j}=t_{p}\end{cases}
$$

where $\mathrm{Z}$ means time interpolation in time $\mathrm{t} \_\mathrm{j}$ and $\mathrm{x} \_\mathrm{i}$ location; $t \_a$ is the first valid data of $x \_i$ location; $t \_1$ is initial moment; $t \_p$ is end time; $x \_i$ is the place where interpolation needs to be performed.

In Figure 3, it can be seen from Figures 3(a) to 3(c) that the distribution of data is relatively discrete, and there are many outliers in each lane in density, the rate of changing into lanes, and the rate of changing out lanes. The $50 \%$ quantile data are all distributed low, which is biased towards the $25 \%$ quantile data. In Figure 3(a), the density distribution in Lane 1 to Lane 3 is relatively similar, and the distribution in Lane 4 is less. The graphs in Figures 3(b) and 3(c) are similar. Lane 1 and Lane 4 have a lower lane change rate, and Lane 2 and Lane 3 have a higher lane change rate. The graph shows that the lane change-out rate of Lane 4 is lower than the lane change-in rate of Lane 4 . This is mainly because Lane 4 is directly connected to the entrance and exit. For vehicles, except for those that must leave the road, they are basically reluctant to drive in this lane, the density of the lane is the lowest, and fewer vehicles are swapped out. Vehicles that have to get out of the road must change into the lane, so the rate of changing into lanes is slightly higher than that of changing out lanes. The situation shown in Figure 3 is basically the same as what actually happened on the road. The statistical analysis of the three parameters can also be further proved. The statistical analysis of the data description is shown in Table 1.

It can be seen from Table 1 that the average density of Lane 1 to Lane 3 is relatively close, and the density of Lane 4 is lower. At the same time, there is a gap between the density mean, the density median, and the density mode. This is reflected in the rate of changing into lanes and the rate of changing out lanes. The data of the three parameters on each lane show that the data is nonnormally distributed. In addition, the rate of changing into lanes and the rate of changing out lanes have lower mean values in Lane 1 and Lane 3, while the mean values of Lane 2 and Lane 3 are both 


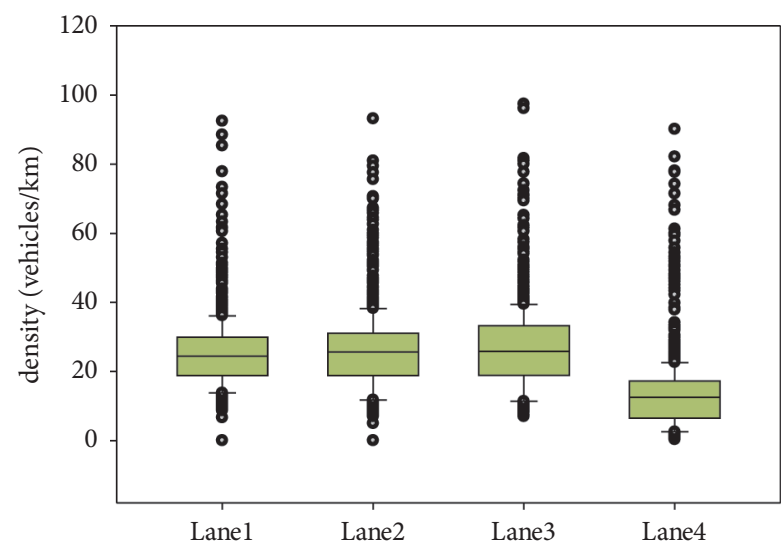

(a)

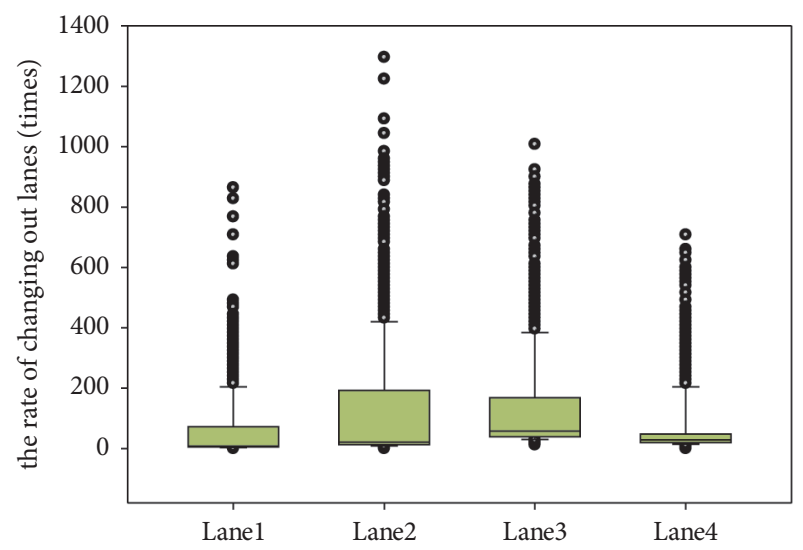

(b)

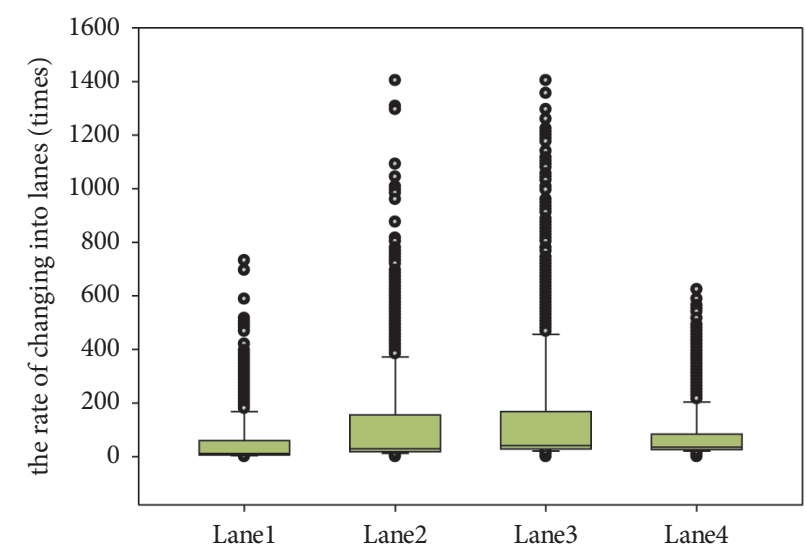

(c)

FIgURE 3: Box plot of empirical data distribution. (a) Box diagram of density distribution of a single lane. (b) A box chart of the rate of changing out lanes of a single lane. (c) A single lane box chart of the rate of changing into lanes.

TABLE 1: Empirical data by the statistical description.

\begin{tabular}{|c|c|c|c|c|c|}
\hline & & Lane 1 & Lane 2 & Lane 3 & Lane 4 \\
\hline \multirow{7}{*}{ Density (vehicle/km) } & Mean value & 25.33149 & 26.14947 & 26.79935 & 13.66627 \\
\hline & Standard error & 0.334813 & 0.375786 & 0.40465 & 0.372006 \\
\hline & Median & 24.44 & 25.6438 & 25.79167 & 12.54167 \\
\hline & Mode & 22.66667 & 25.08333 & 25.79167 & 2.583333 \\
\hline & Variance & 103.4679 & 130.3416 & 151.1339 & 127.7324 \\
\hline & Kurtosis & 6.95363 & 3.987783 & 4.232871 & 10.95704 \\
\hline & Skewness & 1.730317 & 1.305238 & 1.327613 & 2.656881 \\
\hline \multirow{7}{*}{ Rate of changing out lanes (times) } & Mean value & 123.7573 & 277.5991 & 339.7183 & 154.7129 \\
\hline & Standard error & 3.271707 & 6.147944 & 8.187349 & 3.616784 \\
\hline & Median & 108 & 240 & 300 & 132 \\
\hline & Mode & 24 & 144 & 72 & 48 \\
\hline & Variance & 9879.854 & 34886.83 & 61871.17 & 12073.88 \\
\hline & Kurtosis & 3.218006 & 3.836383 & 2.183544 & 1.075749 \\
\hline & Skewness & 1.279292 & 1.438276 & 1.303985 & 1.04637 \\
\hline \multirow{7}{*}{ Rate of changing into lanes (times) } & Mean value & 148.6024 & 326.8732 & 293.2264 & 143.0639 \\
\hline & Standard error & 3.734585 & 6.509294 & 5.754521 & 4.287722 \\
\hline & Median & 120 & 276 & 252 & 120 \\
\hline & Mode & 48 & 132 & 192 & 12 \\
\hline & Variance & 12873.2 & 39108.35 & 30564.69 & 16968.94 \\
\hline & Kurtosis & 4.616732 & 1.158177 & 0.726926 & 1.667461 \\
\hline & Skewness & 1.563167 & 0.993322 & 0.956815 & 1.258795 \\
\hline
\end{tabular}


higher. The performance of the above data is consistent with the box plot display and further confirms that the data presents a nonnormal distribution, and the data distribution conforms to the actual road conditions. The data is true and valid and can be further used for simulation.

\subsection{Simulation Model Establishment and Verification. In} order to study the relationship between lane-changing behavior and density of urban expressways under different penetration rates of autonomous vehicles, the actual urban expressway was compared with the road network simulation model. The model is established based on the 9 road sections in the empirical study. By simplifying the road network, only exits and entrances are retained.

Using SUMO as the simulation platform, the road section is set as a one-way 4-lane. In order to reflect the actual situation of the road more realistically, in the simulation model, each road section has 4 entrances and 1 exit. The road section setting is shown in Figure 4.

In this article, the simulation model is shown in Figure 4. According to the actual situation in the empirical study, four types of vehicles are set as large trucks, medium trucks, cars, and autonomous vehicles. Among them, the autonomous vehicle is set as car. Each section contains 4 entrances and one exit. The distance between the entrance and the exit is $1 \mathrm{~km}$, the simulation distance of each road section is $5 \mathrm{~km}$, and the simulation distance of the whole road is $45 \mathrm{~km}$, which is close to the total length of $51 \mathrm{~km}$ of Chengdu's third ring road. The simulation time is $86400 \mathrm{~s}$, that is, $24 \mathrm{~h}$. The simulation time period is 4 hours in the morning and 4 hours in the afternoon of the working day. The simulation step is $0.1 \mathrm{~s}$. The tranCI interface is used to add different traffic flows to different intersections of the road. The data collection time interval is 5 minutes, and the collected data includes the density, the lane change-in rate, and the lane change-out rate. Data collection was performed on 2591 groups.

Before conducting autonomous vehicles' penetration rate experiment, first the availability of the simulation model is verified. The traffic flow was run which composed of three types of large trucks, medium trucks, and cars in the simulation model, and traffic flow density, the rate of changing into lanes, and the rate of changing out of lanes were collected to compare with the data in the empirical study. Model verification was carried out. The simulation data distribution box diagram is shown in Figure 5.

In Figure 5, from Figures 5(a) to 5(c), the data distribution of density, the rate of changing into lanes, and the rate of changing out lanes are, respectively, shown. From the figure, the density distribution in Figure 5(a) is Lane 4, which is the largest, Lane 2 is the second, and Lane 1 is the smallest distribution. The distributions of the rate of changing into lanes and the rate of changing out lanes in Figures 5(b) and 5(c) are relatively similar. Lane 1 is the smallest, lanes 2 and 3 are larger, and Lane 3 is the busiest. The $50 \%$ quantile of the data shown in Figure 5 is close to the 25\% quantile, indicating that the data distribution is nonnormal. It can also be further confirmed from the simulation data statistics, as shown in Table 2.

It can be seen from Table 2 that the average density of Lane 1 and Lane 3 is lower, and the average density of Lane 4 is the largest. The rate of changing out lanes and the rate of changing into lanes are the smallest in Lane 1, and the remaining three lanes are more balanced, of which Lane 3 is the largest. This is mainly due to the fact that Lane 3 accepts a large number of traffic flow from Lane 1 and Lane 2 that need to leave the road. At the same time, it also accepts the traffic flow from Lane 4, so lane change behavior in Lane 3 occurs more frequently. In addition, the data in the table shows that the average is inconsistent with the median and mode, and the data has a skewness higher than 0.7. Therefore, the data presents a nonnormal distribution. The data of simulation and empirical research differ in distribution. However, we found that the data from the simulation experiment and the empirical research both present a nonnormal distribution. At the same time, the relationship between density and the rate of changing out lanes and the relationship between density and the rate of changing into lanes is relatively close, as shown in Figure 6.

It can be seen from Figure 6 that Figures 6(a), 6(c), 6(e), and $6(\mathrm{~g})$ are empirical data and Figures $6(\mathrm{~b}), 6(\mathrm{~d}), 6(\mathrm{f})$, and $6(\mathrm{~h})$ are the simulation data. The data is concentrated in the lower left corner, and the discrete form is beyond the upper right corner. There are differences between the empirical data and the simulation data, but the relationship characteristics are relatively similar, so we believe that the simulation model can be used to show realistic road conditions.

2.3. Data Sources. In our research, the data was used to analyze the relationship between density and the rate of changing into lanes and the relationship between density and the rate of changing out lanes. The data from videos and simulation model were used in this paper. Most of the videos were captured from live shooting, and part of them were from the Chengdu Traffic Management Bureau. Data was collected data from video via the traffic data collection software and manual work. And then, the data was cleaned and complemented before analyzed. In this research, the data of density, the rate of changing out lanes, and the rate of changing into lanes in different lane was used. The data can be accessed from our team, only be allowed in research. Because of some data came from Bureau, only desensitized data can be proved. The part of data we used is shown in Table 3. In Table 3, K1 means density of Lane 1, K2 means density of Lane 2, LCol means the rate of changing out lanes of Lane 1, LCo2 means the rate of changing out lanes of Lane 2, LCi1 means the rate of changing into lanes of Lane 1, and LCi2 means the rate of changing into lanes of Lane 2. 


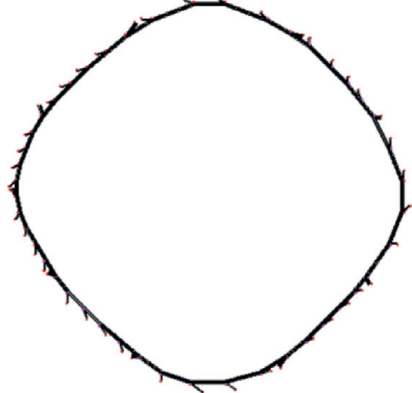

(a)

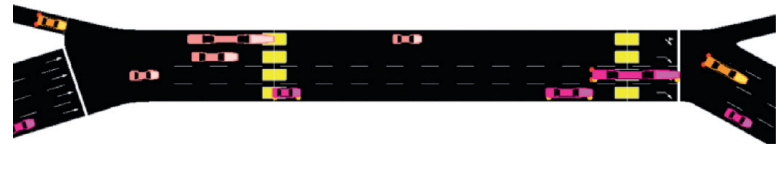

(b)

Figure 4: Schematic diagram of simulation modeling. (a) Road network model established by simulation. (b) A road running diagram with one exit, one entrance, and two detectors.

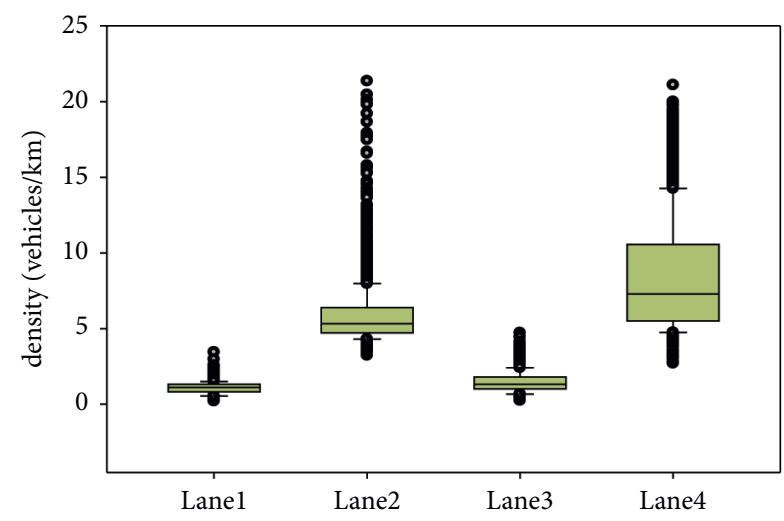

(a)

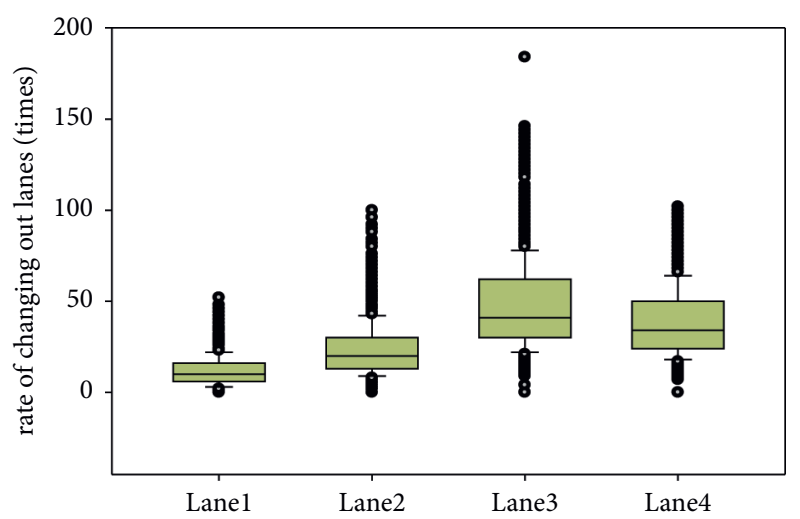

(b)

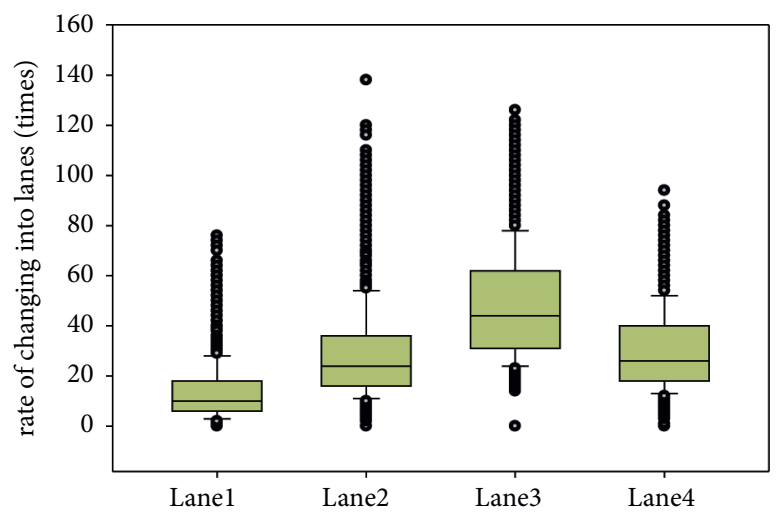

(c)

Figure 5: Box diagram of simulation data distribution. (a) A box diagram of the density distribution of a single lane. (b) A box chart of the rate of changing out lanes of a single lane. (c) A box chart of the rate of changing into lanes of a single lane.

\section{Simulation Experiment and Results}

3.1. Design of Simulation Experiments for the Penetration Rate of Different Autonomous Vehicles. Through the previous verification, the simulation model and the research object in the empirical study have a certain similarity in the relationship between density and lane-changing behavior. Therefore, the simulation model can be used to study the relationship between density and lane-changing behavior of autonomous vehicles under different permeability. The addition of autonomous vehicles will have a certain impact on density distribution and lane-changing behavior. For example, autonomous vehicles will automatically choose to follow the car when the speed is currently maintained at the optimal situation, instead of actively changing lanes, and will be in front of the lane. In the case of impossibility or only changing lanes to get out of the road, the lane will definitely be changed actively, and the behavior of changing lanes will have a certain impact on the density of each lane. Therefore, studying the relationship between density and lane-changing 
Table 2: Simulation data by the statistical description.

\begin{tabular}{|c|c|c|c|c|c|}
\hline & & Lane 1 & Lane 2 & Lane 3 & Lane 4 \\
\hline \multirow{7}{*}{ Density (vehicle/km) } & Mean value & 1.075899 & 5.900002 & 1.457771 & 8.466858 \\
\hline & Standard error & 0.007279 & 0.039991 & 0.013194 & 0.072797 \\
\hline & Median & 1.11 & 5.33 & 1.32 & 7.28 \\
\hline & Mode & 1.17 & 5.35 & 1.295 & 4.8 \\
\hline & Variance & 0.137273 & 4.14377 & 0.451078 & 13.73066 \\
\hline & Kurtosis & 1.165419 & 12.17008 & 1.090342 & 0.184097 \\
\hline & Skewness & 0.325542 & 2.920906 & 0.981555 & 1.00062 \\
\hline \multirow{7}{*}{ Rate of changing out lanes (times) } & Mean value & 11.76071 & 23.35006 & 47.30066 & 38.01775 \\
\hline & Standard error & 0.158519 & 0.280317 & 0.458097 & 0.342657 \\
\hline & Median & 10 & 20 & 42 & 34 \\
\hline & Mode & 8 & 18 & 36 & 24 \\
\hline & Variance & 65.1072 & 203.5944 & 543.7285 & 304.219 \\
\hline & Kurtosis & 1.640203 & 2.948731 & 1.678656 & 0.115027 \\
\hline & Skewness & 1.179921 & 1.461843 & 1.128802 & 0.797563 \\
\hline \multirow{7}{*}{ Rate of changing into lanes (times) } & Mean value & 13.05558 & 29.15515 & 48.31223 & 29.90621 \\
\hline & Standard error & 0.21225 & 0.363476 & 0.414709 & 0.298989 \\
\hline & Median & 10 & 24 & 44 & 26 \\
\hline & Mode & 8 & 28 & 52 & 24 \\
\hline & Variance & 116.7251 & 342.3103 & 445.6102 & 231.6209 \\
\hline & Kurtosis & 3.971726 & 2.844836 & 0.08808 & 0.28378 \\
\hline & Skewness & 1.729006 & 1.485479 & 0.784475 & 0.80799 \\
\hline
\end{tabular}
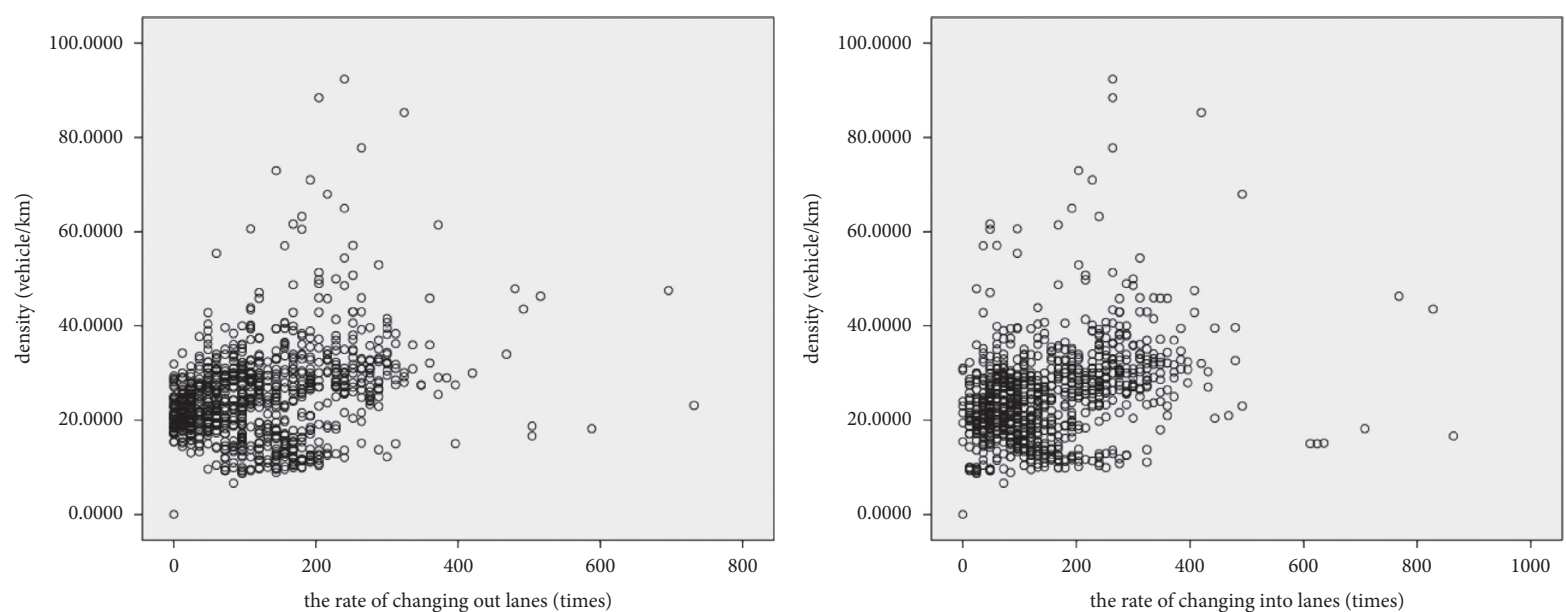

(a)
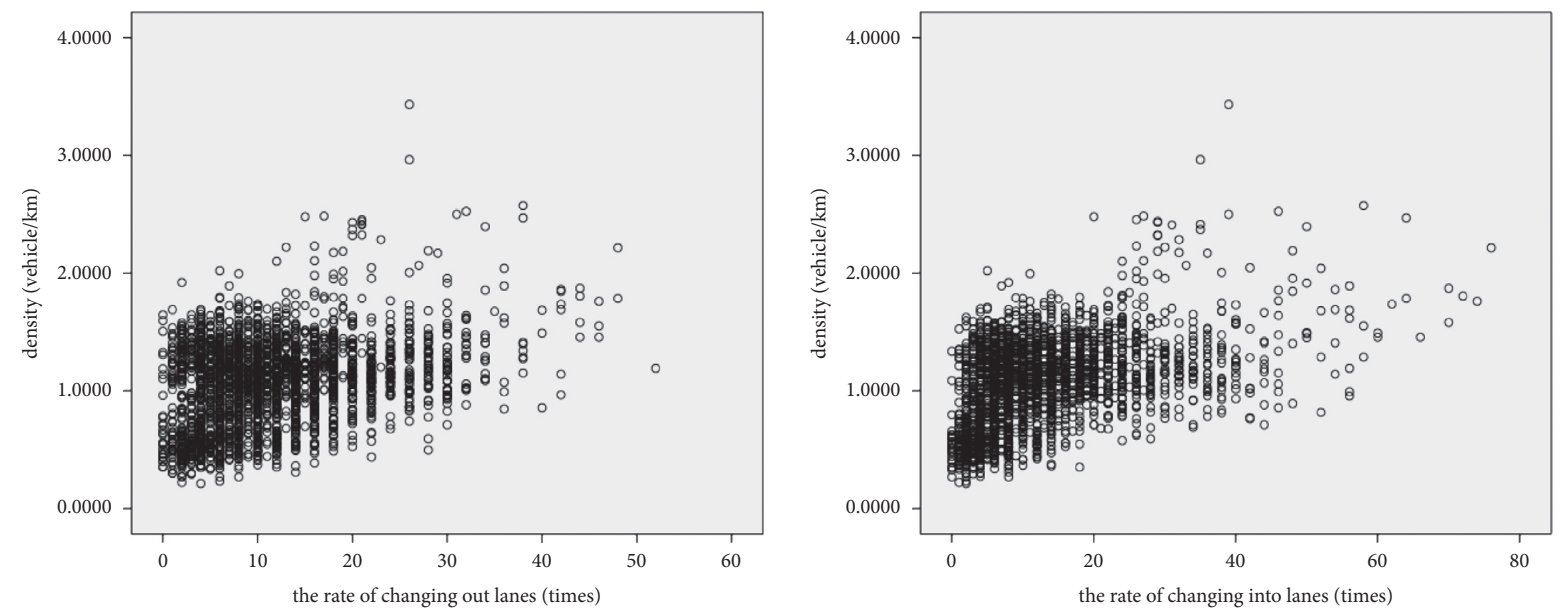

(b)

FIgURE 6: Continued. 

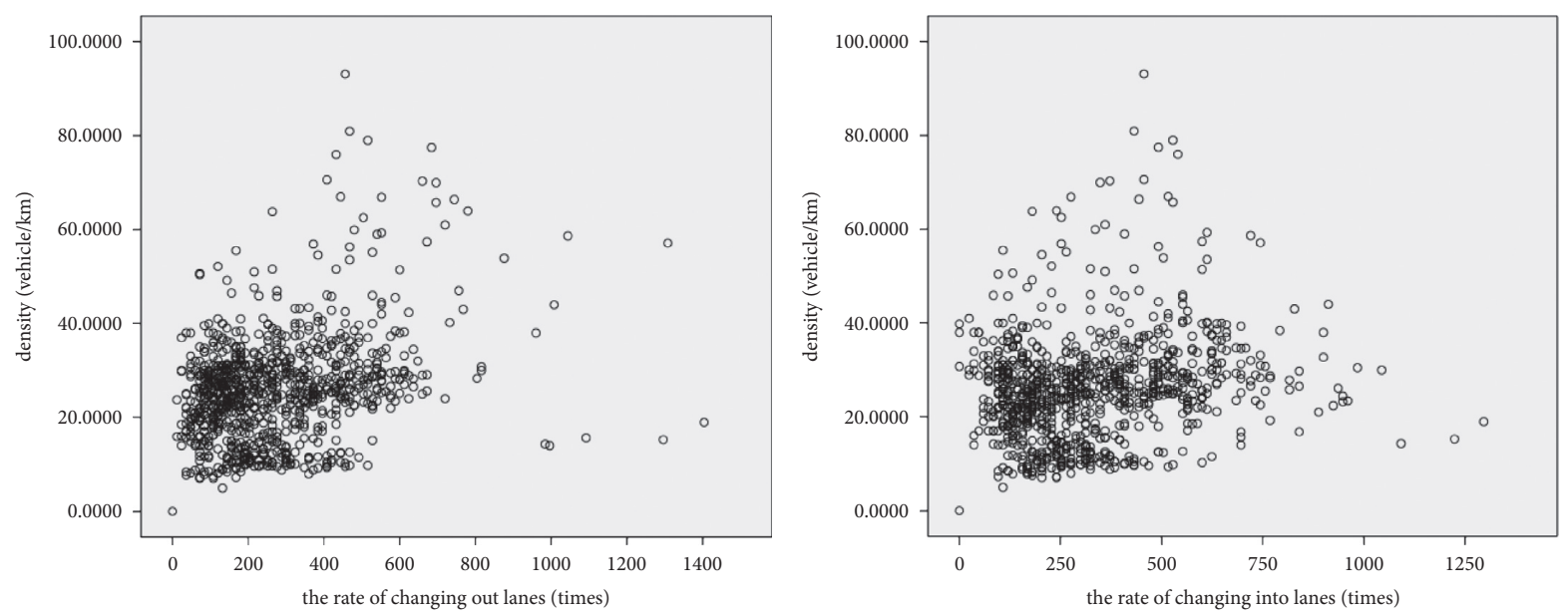

(c)
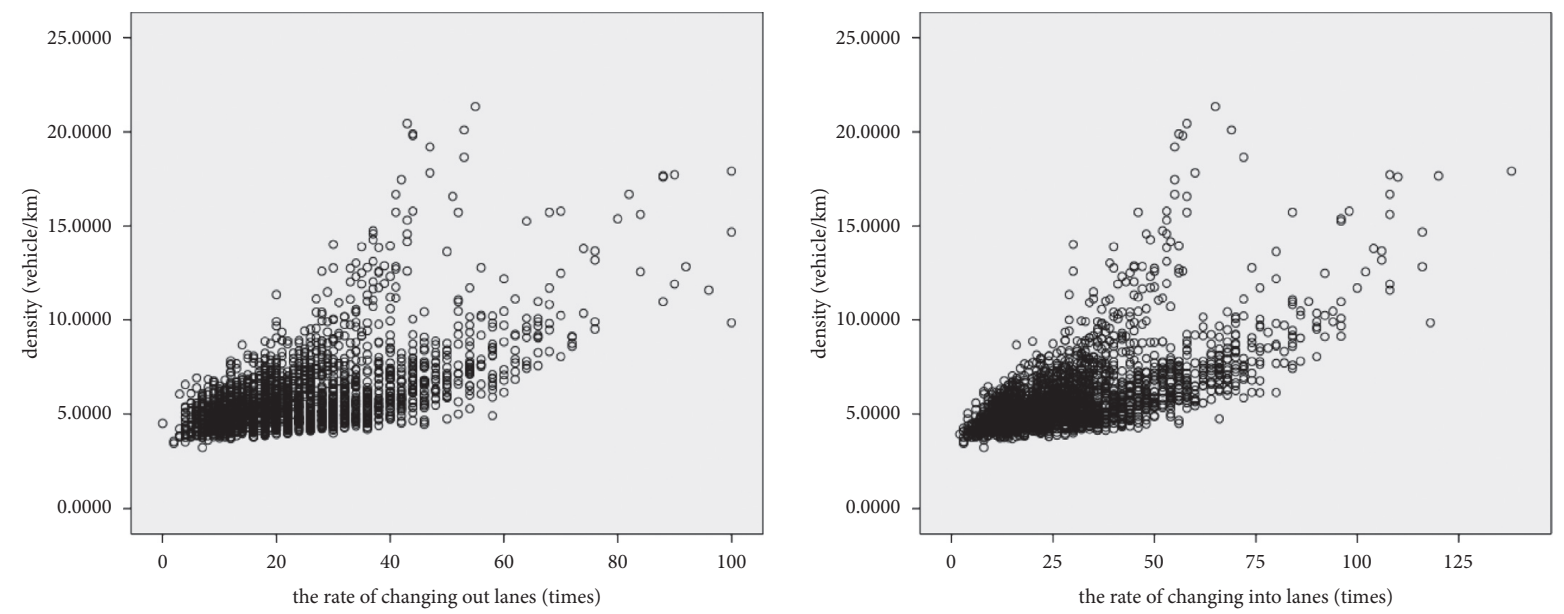

(d)
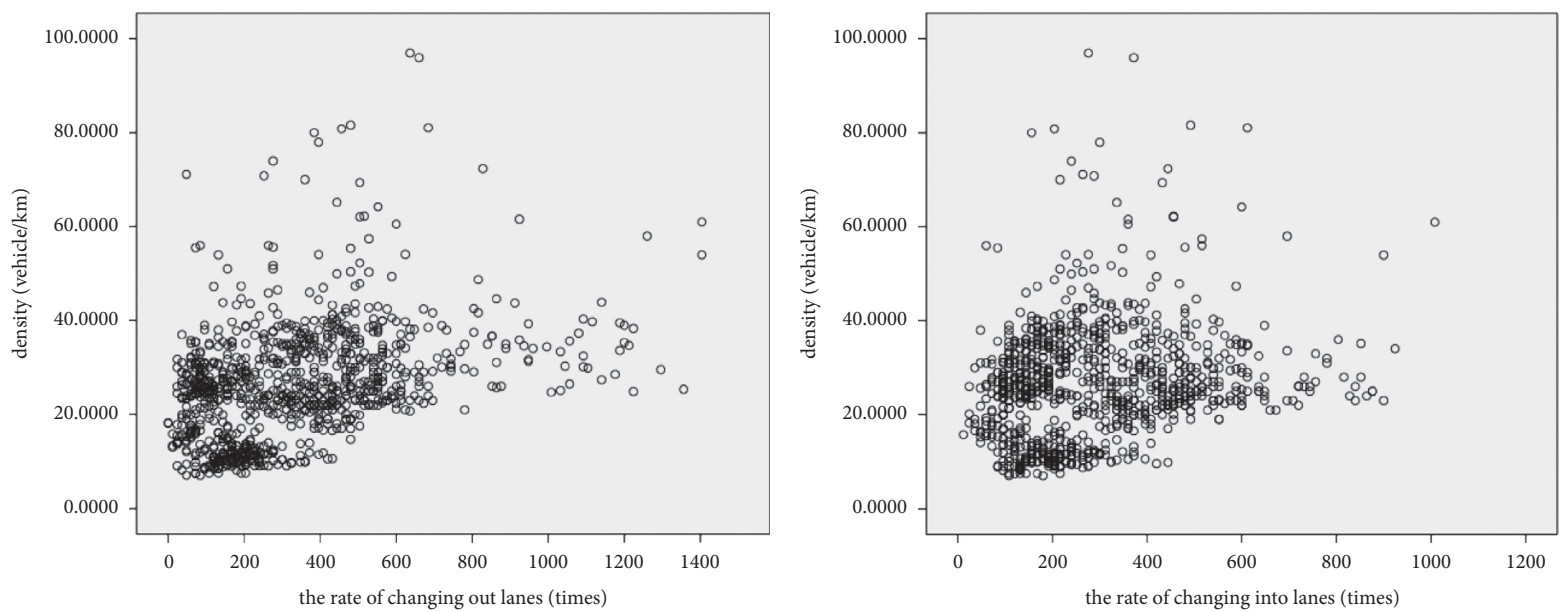

(e)

FIgURE 6: Continued. 

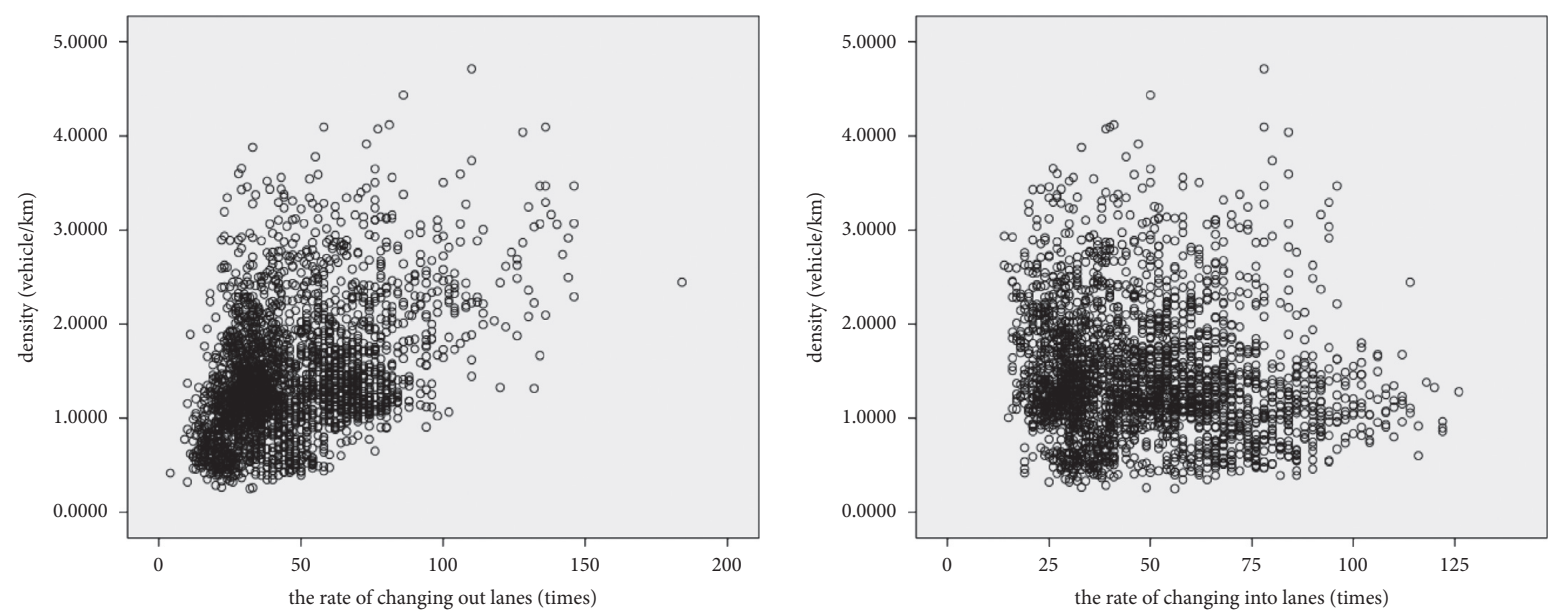

(f)
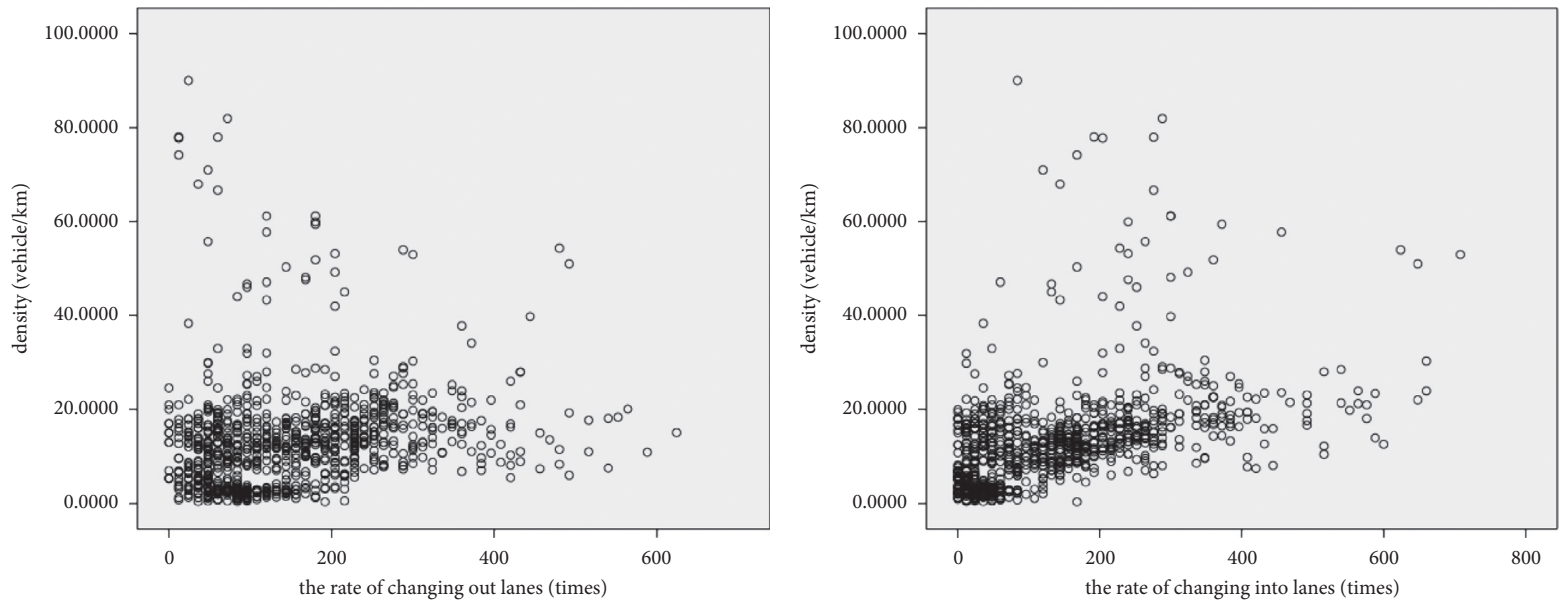

(g)
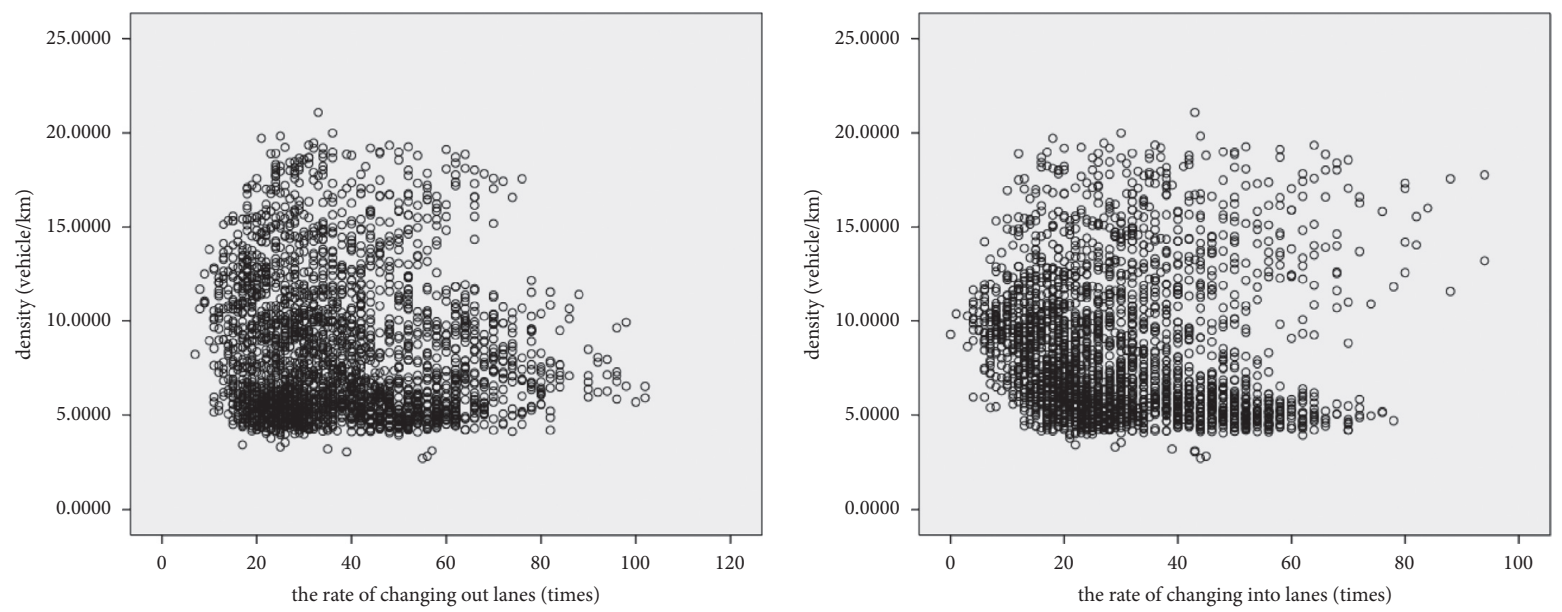

(h)

FIGURE 6: Scatter diagram of empirical and simulation models for the relationship between density and the rate of changing out lanes and between density and the rate of changing into lanes of a single lane. (a) The empirical data of Lane 1. (b) The simulation data of Lane 1. (c) The empirical data of Lane 2. (d) The simulation data of Lane 2. (e) The empirical data of Lane 3. (f) The simulation data of Lane 3. (g) The empirical data of Lane 4 . (h) The simulation data of Lane 4. 
TABLe 3: The data of density and the number of lane-changing times of Lane 1 and Lane 2.

\begin{tabular}{|c|c|c|c|c|c|}
\hline k1 (vehicle/km) & k2 (vehicle/km) & LCo1 (times) & LCo2 (times) & LCi1 (times) & LCi2 (times) \\
\hline 41.3534 & 49.397 & 276 & 732 & 420 & 588 \\
\hline 45.8286 & 57.419 & 216 & 672 & 276 & 600 \\
\hline 45.9102 & 51.4407 & 360 & 600 & 348 & 600 \\
\hline 51.3625 & 65.8116 & 204 & 696 & 264 & 528 \\
\hline 46.316 & 58.6692 & 516 & 1044 & 768 & 720 \\
\hline 43.6069 & 57.1446 & 492 & 1308 & 828 & 744 \\
\hline 54.4434 & 66.4226 & 240 & 744 & 312 & 444 \\
\hline 47.5244 & 59.9603 & 696 & 480 & 408 & 336 \\
\hline 32.8996 & 46.9474 & 216 & 276 & 180 & 444 \\
\hline 32.6967 & 40.62 & 108 & 396 & 156 & 564 \\
\hline 39.4808 & 42.3747 & 156 & 624 & 384 & 396 \\
\hline 25.3695 & 26.1902 & 144 & 384 & 192 & 300 \\
\hline 29.7359 & 36.3863 & 108 & 492 & 300 & 360 \\
\hline 31.3534 & 38.9231 & 252 & 396 & 228 & 504 \\
\hline 23.1025 & 44.1669 & 156 & 1008 & 492 & 912 \\
\hline 21.307 & 43.1977 & 192 & 768 & 360 & 828 \\
\hline 22.7805 & 38.1319 & 204 & 960 & 360 & 900 \\
\hline 23.1025 & 36.3308 & 84 & 252 & 144 & 408 \\
\hline 23.7316 & 43.2011 & 96 & 336 & 192 & 252 \\
\hline 26.0077 & 45.7935 & 48 & 420 & 240 & 408 \\
\hline 22.6332 & 35.1054 & 12 & 168 & 60 & 132 \\
\hline 20.5373 & 25.0703 & 48 & 120 & 120 & 276 \\
\hline 30.3718 & 39.5103 & 12 & 96 & 24 & 192 \\
\hline 23.2872 & 42.0075 & 60 & 552 & 288 & 516 \\
\hline 20.4341 & 31.7524 & 60 & 540 & 300 & 528 \\
\hline 25.9159 & 33.3484 & 12 & 168 & 84 & 156 \\
\hline 20.6745 & 30.2142 & 24 & 24 & 12 & 108 \\
\hline 23.1988 & 35.4795 & 12 & 72 & 36 & 192 \\
\hline 23.3655 & 37.1458 & 60 & 384 & 180 & 276 \\
\hline 22.4643 & 38.4357 & 60 & 180 & 96 & 324 \\
\hline 50.7629 & 56.3251 & 252 & 468 & 216 & 492 \\
\hline 61.4895 & 59.3006 & 372 & 552 & 168 & 612 \\
\hline 77.8395 & 70.6239 & 264 & 408 & 264 & 456 \\
\hline 85.3344 & 77.5126 & 324 & 684 & 420 & 492 \\
\hline 65.3061 & 67.3181 & 240 & 444 & 192 & 516 \\
\hline 68.3836 & 69.9785 & 216 & 696 & 492 & 348 \\
\hline 71.4634 & 75.5906 & 192 & 432 & 228 & 540 \\
\hline 73.3314 & 79.4491 & 144 & 516 & 204 & 528 \\
\hline 92.4337 & 80.9599 & 240 & 468 & 264 & 432 \\
\hline 88.489 & 93.1523 & 204 & 456 & 264 & 456 \\
\hline 23.5978 & 31.4044 & 24 & 108 & 48 & 96 \\
\hline 24.5965 & 34.4922 & 36 & 132 & 48 & 96 \\
\hline 21.9508 & 31.8681 & 36 & 72 & 36 & 72 \\
\hline 18.5071 & 30.3185 & 0 & 60 & 24 & 24 \\
\hline 22.5266 & 34.0184 & 0 & 84 & 0 & 36 \\
\hline 25.2426 & 40.8139 & 0 & 120 & 48 & 24 \\
\hline 23.99 & 38.4165 & 24 & 36 & 24 & 48 \\
\hline 23.5253 & 38.1263 & 0 & 108 & 0 & 48 \\
\hline 28.4573 & 38.2759 & 0 & 96 & 24 & 0 \\
\hline 22.8712 & 36.2333 & 12 & 108 & 48 & 60 \\
\hline 21.2639 & 46.638 & 192 & 756 & 468 & 384 \\
\hline 40.0034 & 64.448 & 168 & 780 & 276 & 240 \\
\hline 33.9265 & 60.908 & 300 & 720 & 312 & 360 \\
\hline 31.8102 & 59.3811 & 300 & 540 & 324 & 408 \\
\hline 21.7264 & 38.3303 & 12 & 48 & 36 & 36 \\
\hline 19.7166 & 29.0354 & 48 & 264 & 84 & 324 \\
\hline 17.793 & 28.6409 & 12 & 336 & 84 & 288 \\
\hline 17.6955 & 30.2176 & 0 & 216 & 24 & 420 \\
\hline 16.6629 & 26.9723 & 24 & 204 & 96 & 408 \\
\hline 19.8912 & 29.3868 & 12 & 264 & 108 & 336 \\
\hline
\end{tabular}


TABle 3: Continued.

\begin{tabular}{|c|c|c|c|c|c|}
\hline k1 (vehicle/km) & k2 (vehicle/km) & LCo1 (times) & LCo2 (times) & LCi1 (times) & LCi2 (times) \\
\hline 19.0909 & 29.4854 & 48 & 336 & 156 & 372 \\
\hline 19.6112 & 32.2682 & 0 & 360 & 144 & 420 \\
\hline 18.6976 & 33.3303 & 12 & 444 & 192 & 360 \\
\hline 18.4969 & 30.6064 & 48 & 288 & 60 & 480 \\
\hline 20.8796 & 34.329 & 12 & 276 & 132 & 528 \\
\hline 21.9531 & 34.1578 & 36 & 300 & 84 & 576 \\
\hline 17.6105 & 30.9374 & 0 & 360 & 108 & 324 \\
\hline 20.6801 & 35.3208 & 12 & 348 & 120 & 384 \\
\hline 22.1571 & 37.4518 & 12 & 324 & 120 & 372 \\
\hline 21.2866 & 34.4638 & 48 & 384 & 132 & 480 \\
\hline 22.6139 & 36.7649 & 24 & 396 & 48 & 456 \\
\hline 21.5212 & 36.7411 & 36 & 444 & 132 & 348 \\
\hline 24.584 & 41.1188 & 36 & 240 & 84 & 516 \\
\hline 21.808 & 38.5786 & 48 & 276 & 84 & 636 \\
\hline 20.0102 & 35.5554 & 36 & 420 & 192 & 408 \\
\hline 18.599 & 33.0537 & 24 & 348 & 192 & 396 \\
\hline 19.9377 & 35.204 & 24 & 420 & 156 & 504 \\
\hline 20.2188 & 31.3829 & 0 & 264 & 96 & 576 \\
\hline 20.2131 & 36.3557 & 24 & 432 & 96 & 480 \\
\hline 22.0177 & 31.7434 & 60 & 420 & 156 & 456 \\
\hline 23.9129 & 36.3455 & 48 & 420 & 108 & 648 \\
\hline 25.5588 & 23.1977 & 144 & 456 & 288 & 948 \\
\hline 26.5212 & 23.3961 & 96 & 468 & 228 & 960 \\
\hline 25.8422 & 24.4366 & 96 & 456 & 252 & 948 \\
\hline 25.0057 & 22.5448 & 132 & 528 & 312 & 744 \\
\hline 19.4831 & 31.5144 & 12 & 228 & 84 & 648 \\
\hline 20.781 & 35.4636 & 60 & 276 & 60 & 444 \\
\hline 23.0027 & 39.3199 & 12 & 324 & 48 & 696 \\
\hline 22.1072 & 34.7121 & 48 & 336 & 84 & 684 \\
\hline 19.6293 & 34.1419 & 48 & 240 & 36 & 660 \\
\hline 19.6803 & 31.2469 & 24 & 192 & 72 & 696 \\
\hline 21.1879 & 34.7733 & 48 & 252 & 60 & 408 \\
\hline 22.8225 & 37.6026 & 0 & 336 & 60 & 624 \\
\hline 22.6615 & 34.0784 & 72 & 360 & 108 & 624 \\
\hline 19.5182 & 33.7168 & 60 & 276 & 48 & 588 \\
\hline 17.0642 & 37.3883 & 24 & 252 & 12 & 468 \\
\hline 18.7214 & 39.8209 & 504 & 252 & 12 & 0 \\
\hline 19.5749 & 41.3942 & 24 & 384 & 96 & 480 \\
\hline 18.3122 & 35.9703 & 12 & 288 & 120 & 444 \\
\hline
\end{tabular}

behavior under different self-driving vehicle penetration rates is very important for understanding the traffic operation mechanism of mixed traffic. In the simulation process, we use the CACC vehicle to simulate the self-driving vehicle, and the remaining settings of the simulation environment remain unchanged. The simulation experiment adopts 8 states that the CACC vehicle penetration rate is designed to be $10 \%, 20 \%, 30 \%, 40 \%, 50 \%, 60 \%, 70 \%$, and $80 \%$. Among them, the permeability is represented by $\mathrm{P}$. The simulation collects the traffic density, the rate of changing into lanes, and the rate of changing out lanes data of each lane at a time interval of 5 minutes and analyzes them. Finally, the relationship between density and lane-changing behavior in the mixed traffic is obtained.

3.2. Permeability Simulation Experiment Results of Different Autonomous Vehicles. After 8 rounds of experiments, we obtained the data of density, the rate of changing out lanes, and the rate of changing into lanes under the penetration rate of autonomous vehicles is $10 \%, 20 \%, 30 \%, 40 \%, 50 \%$, $60 \%, 70 \%$, and $80 \%$. For a better comparison, the 8 -round test data is compared with the data without autonomous vehicles. The data distribution is shown in Figures 7-9.

In Figure 7, with the penetration of autonomous vehicles, the density still shows more outliers, but the number decreases with the addition of autonomous vehicles. In $25 \%$ and $75 \%$ quantile of density, as the penetration rate of autonomous vehicles increases, Lane 1 to Lane 3 are gradually approaching, but Lane 4 increases with the increase in penetration rate. The $50 \%$ quantile of density is still close to the $25 \%$ quantile. The average density of Lane 1 is still the smallest, and the average density of Lane 4 is still the largest. From the state shown in Figure 7, the addition of autonomous vehicles has a certain improvement in the density of lanes from Lane 1 to Lane 3, while Lane 4 is not obvious and even has an increasing trend. 


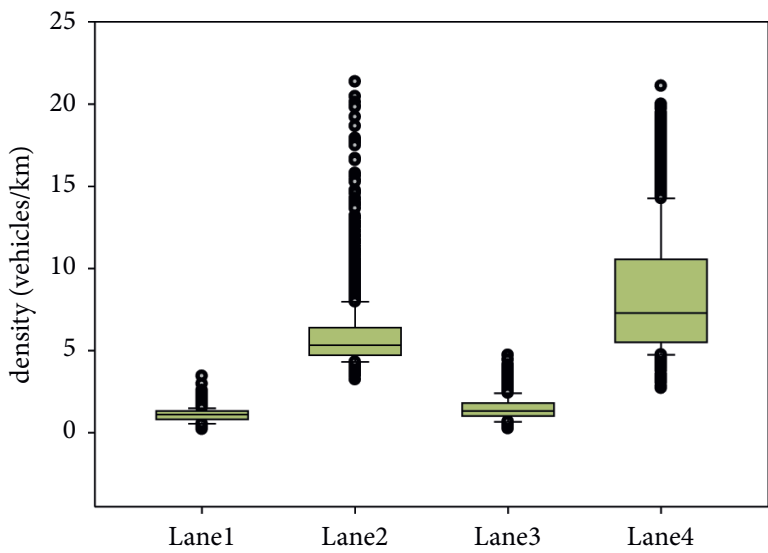

(a)

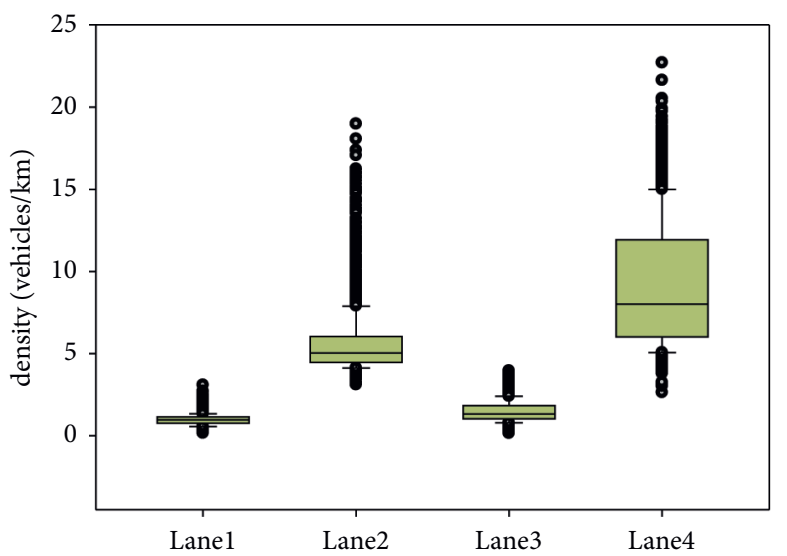

(c)

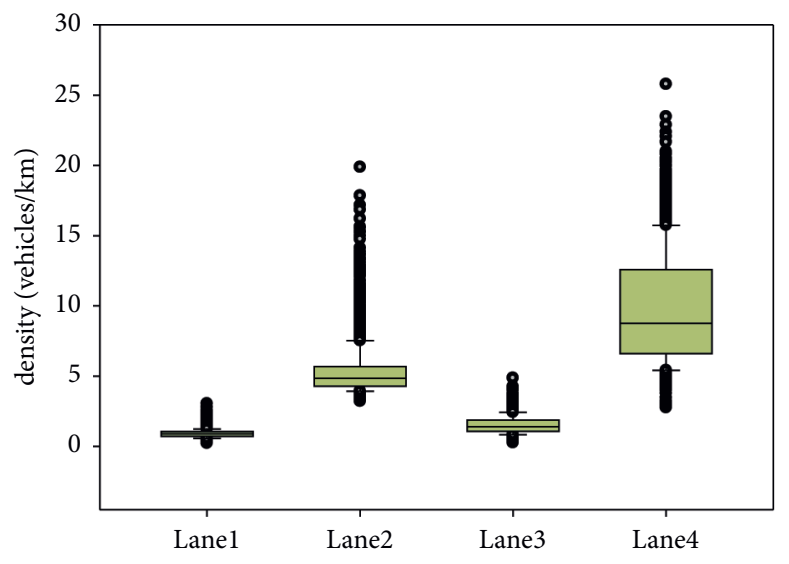

(e)

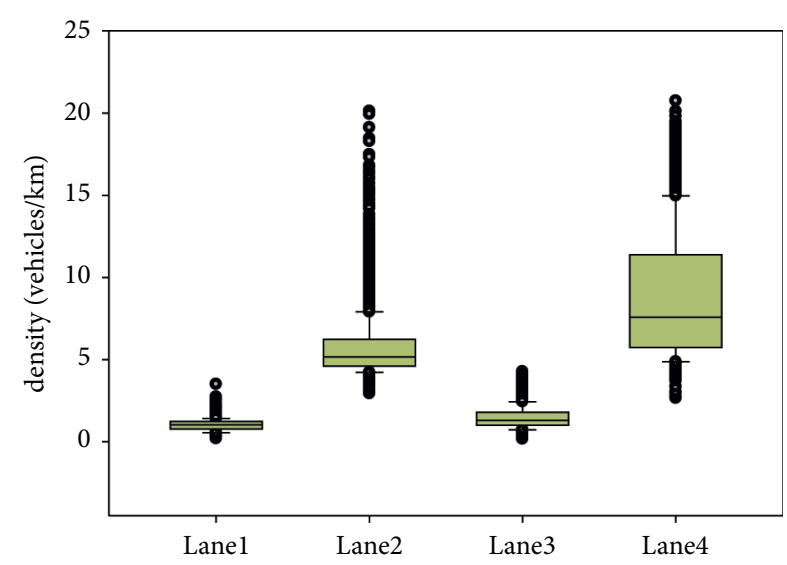

(b)

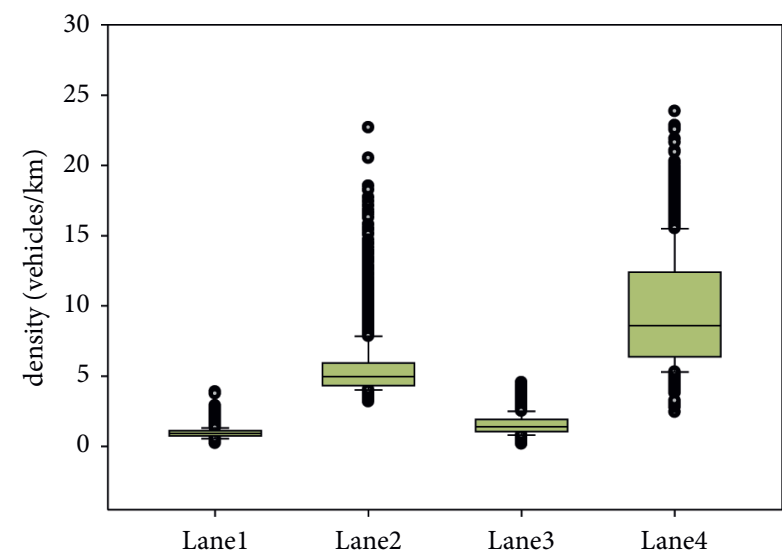

(d)

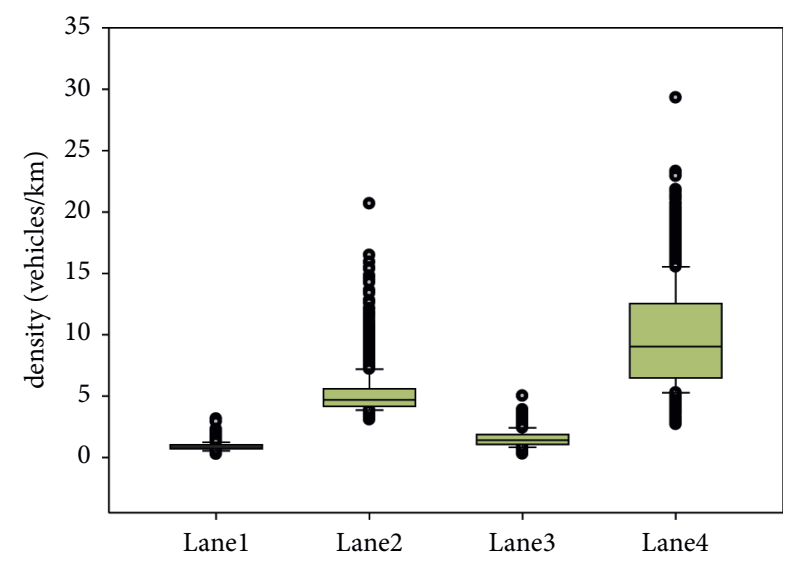

(f)

Figure 7: Continued. 


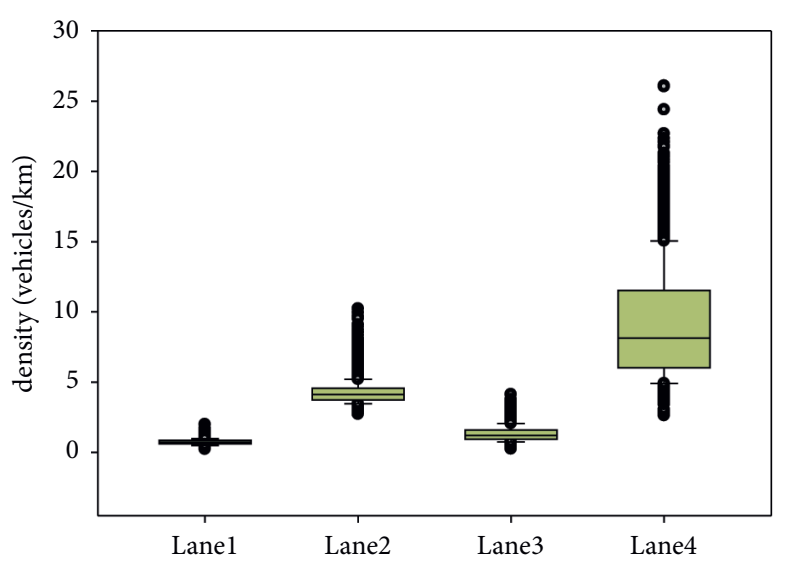

(g)

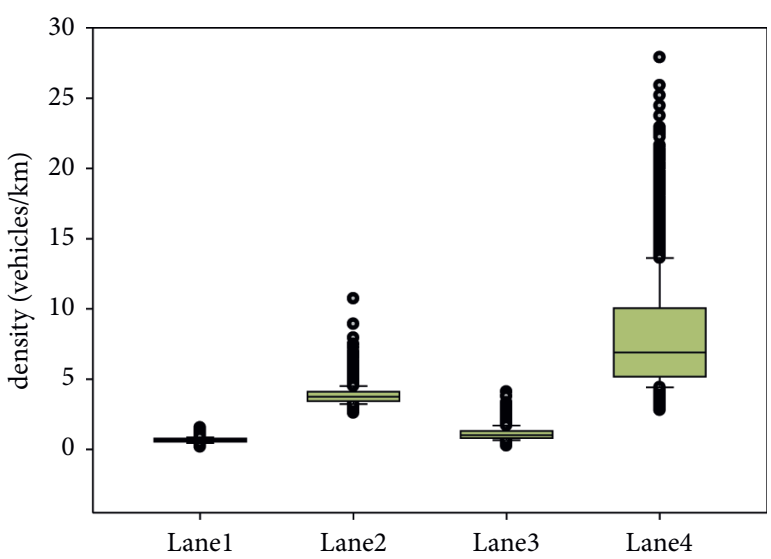

(h)

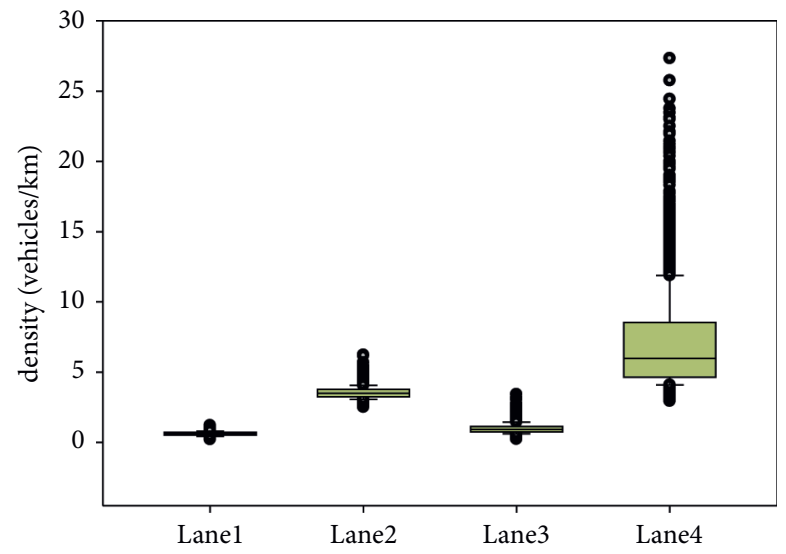

(i)

FiguRE 7: The density distribution of a single lane under different autonomous vehicle penetration rates. The lane density distribution map (a) for the $0 \%$ joining rate of autonomous vehicles, (b) for the $10 \%$ joining rate of autonomous vehicles, (c) for the $20 \%$ joining rate of autonomous vehicles, (d) for the $30 \%$ joining rate of autonomous vehicles, (e) for the $40 \%$ joining rate of autonomous vehicles, (f) for the $50 \%$ joining rate of autonomous vehicles, (g) for the $60 \%$ joining rate of autonomous vehicles, $(\mathrm{h}$ ) for the $70 \%$ joining rate of autonomous vehicles, and (i) for the $80 \%$ joining rate of autonomous vehicles.

Figures 8 and 9 are generally close in performance. With the penetration of autonomous vehicles, there are still many outliers in the data. In Figure 8, the data distribution in $25 \%$ and $75 \%$ is relatively close. There is no significant difference. At the same time, the $50 \%$ quantile is still close to the $25 \%$ quantile. In Figure 8, compared to the other three lanes, the rate of changing into lanes of Lane 1 has a large gap in both the mean value and interval. In Figure 9, the gap between Lane 1 and the other three lanes is not as obvious as in Figure 8 . This is mainly because, compared to Lane 1 , most vehicles will be less affected by the entrance and exit because Lane 1 is farther away. At the same time, Lane 1 is less dense and less traffic pressure, so more choose to change into this lane, unless necessary unwilling to leave the lane. The above analysis can be further verified from Tables 4 to 6 of the statistical description data.

In Table 4, the data representations of Lane 1 and Lane 2 are relatively close. The average density decreases slightly with the increase in the penetration rate of autonomous vehicles. Before the penetration rate is $60 \%$, as the penetration rate increases. The density drops from $1 \%$ to $5 \%$, but at $60 \%$, the density drops by more than $17 \%$, at $70 \%$, it drops slightly, and at $80 \%$, it is slightly lower than $70 \%$. As the penetration rate in Lane 3 increases, the density increases and then decreases before $60 \%$ and suddenly decreases when it reaches $60 \%$, and the degree of $70 \%$ decrease increases, and $80 \%$ decreases slightly from $70 \%$. Lane 4 has an increase in density with the penetration of autonomous vehicles. The increase reaches the highest penetration rate at $30 \%$ and then begins to decrease at $40 \%$, but the density increases compared to $30 \%$, until $60 \%$. It begins to decline, and the rate of decline increases with the increase of permeability, but the rate of decrease of density at $80 \%$ is less than that at $70 \%$. However, changes in the average density data show that when the penetration rate of autonomous vehicles reaches $70 \%$, the density of all lanes decreases compared with the situation without the autonomous vehicles. The density median and density mode decrease slightly in Lane 1 and Lane 2 and have a similar trend, while those in Lane 3 and Lane 4 increase and decrease and then decrease after the $60 \%$ quantile. The $75 \%$ quantile density decreases with the penetration of autonomous vehicles from 


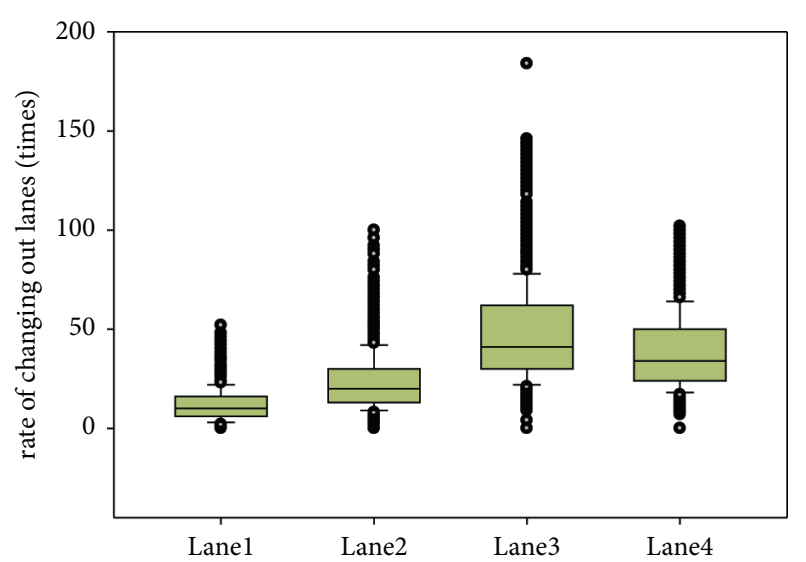

(a)

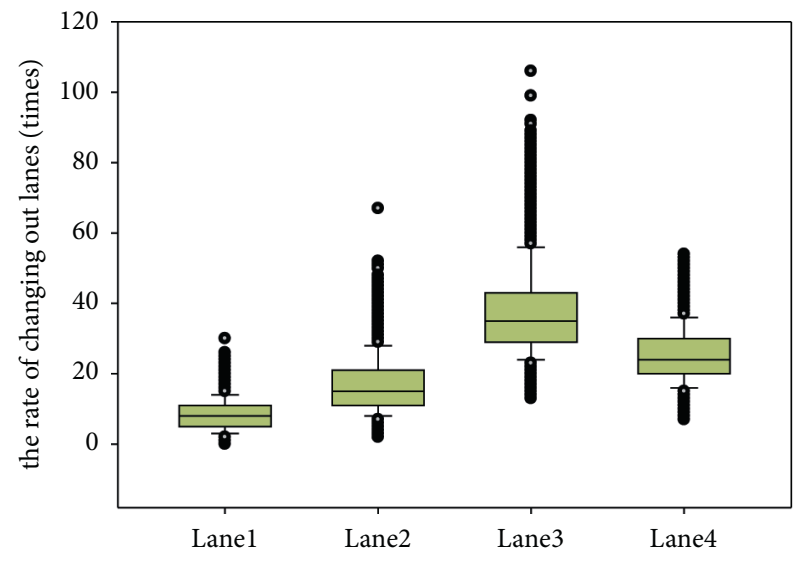

(c)

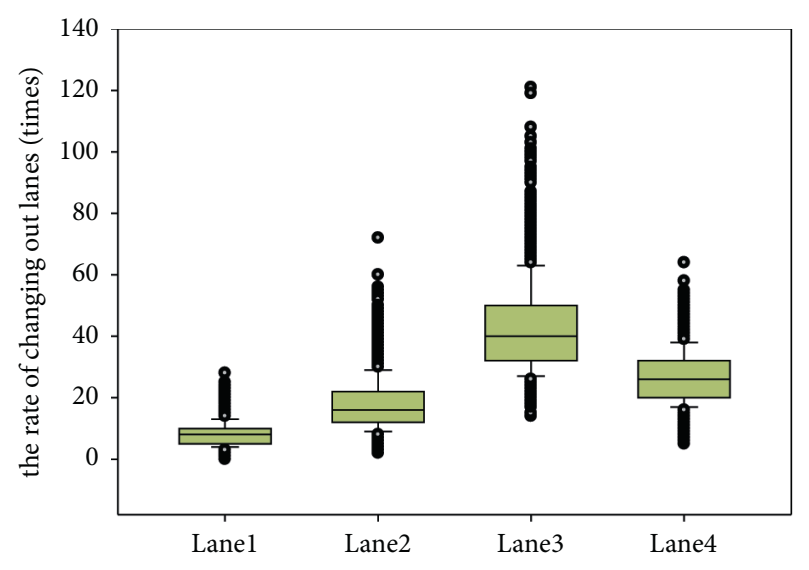

(e)

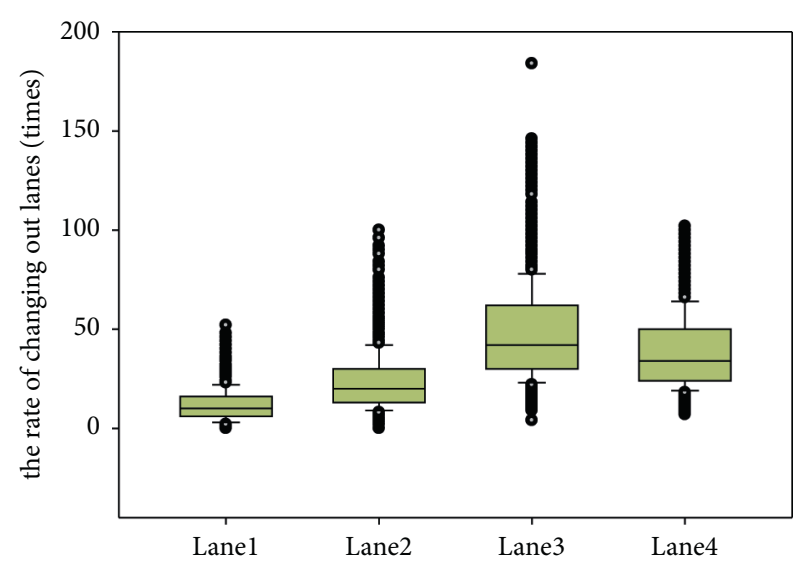

(b)

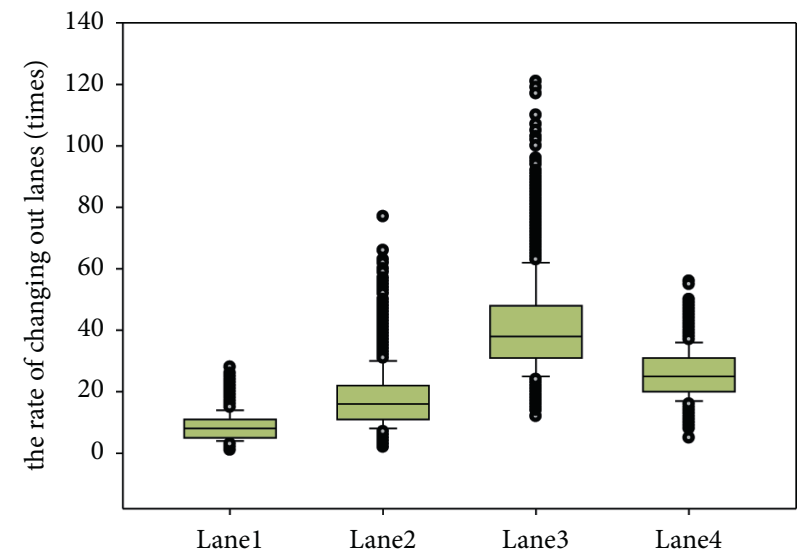

(d)

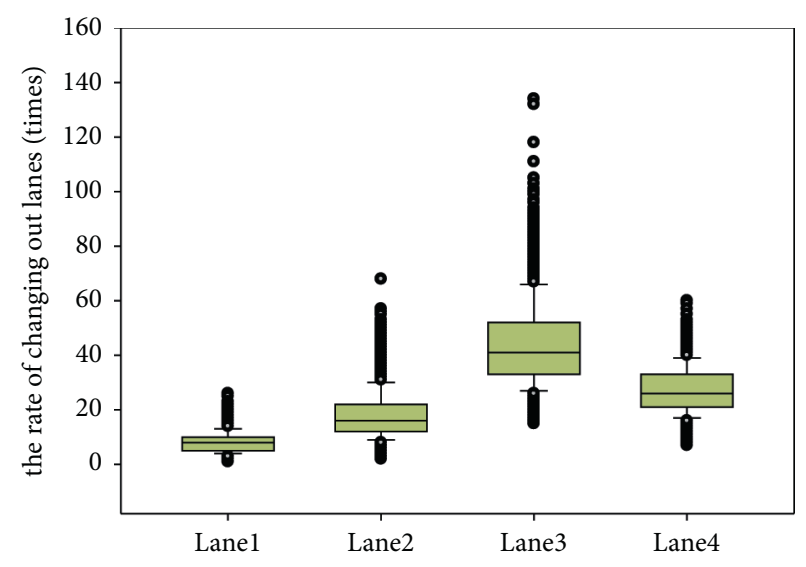

(f)

Figure 8: Continued. 


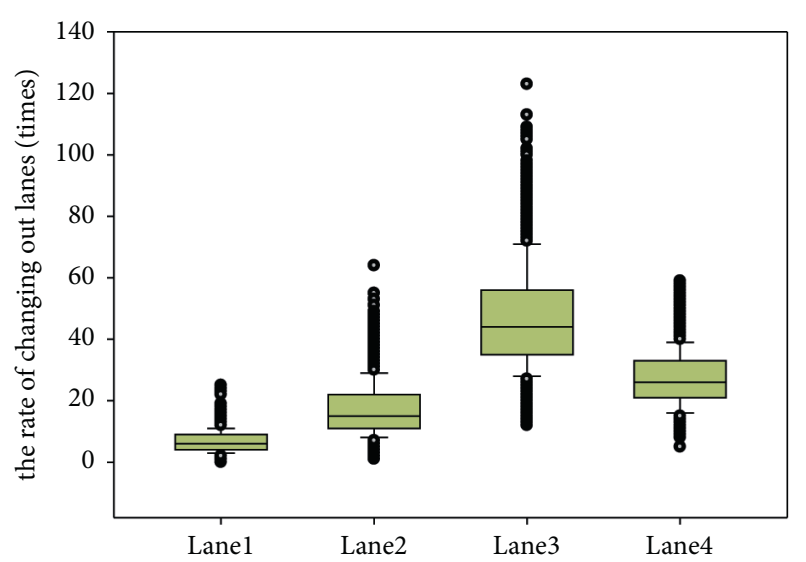

(g)

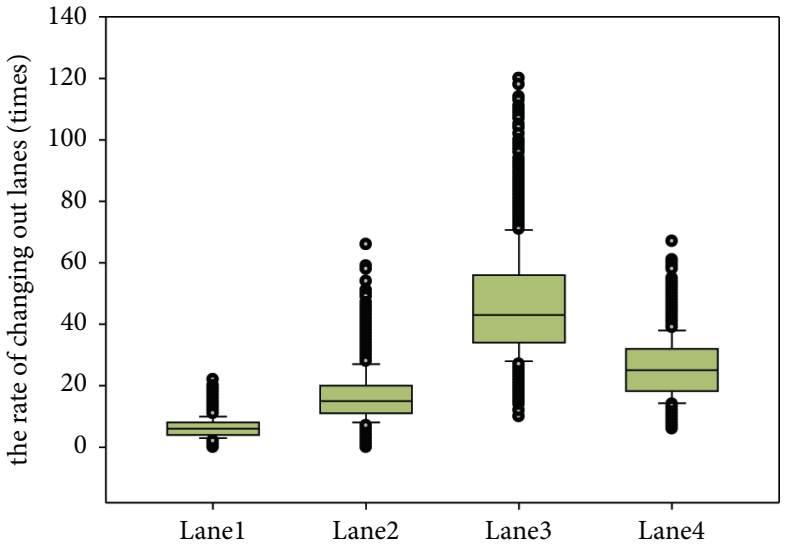

(h)

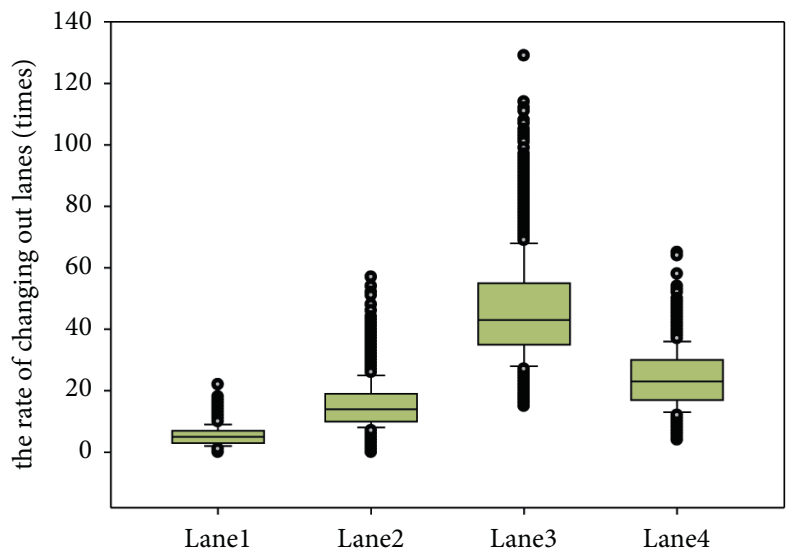

(i)

FIgURE 8: Distribution of the rate of changing out lanes for a single lane under different penetration rates of autonomous vehicles. Distribution map of the rate of changing out lanes (a) for the $0 \%$ joining rate of autonomous vehicles, (b) for the $10 \%$ joining rate of autonomous vehicles, (c) for the $20 \%$ joining rate of autonomous vehicles, (d) for the $30 \%$ joining rate of autonomous vehicles, (e) for the $40 \%$ joining rate of autonomous vehicles, (f) for the $50 \%$ joining rate of autonomous vehicles, (g) for the $60 \%$ joining rate of autonomous vehicles, (h) for the $70 \%$ joining rate of autonomous vehicles, and (i) for the $80 \%$ joining rate of autonomous vehicles.

Lane 1, while Lane 4 has increased or decreased with little change. The $25 \%$ quantile density increased slightly in Lane 1 and Lane 2 with little change, while that in Lane 3 and Lane 4 increased and decreased. However, both the $25 \%$ and $75 \%$ quantile densities converge towards the mode. Taking a comprehensive look at the density in Table 4, the average density from Lane 1 and Lane 2 decreases with the penetration of autonomous vehicles, while that from Lane 3 and Lane 4 only begins to decrease significantly when the penetration rate reaches $60 \%$. But when the penetration rate reaches $80 \%$, the density of all lanes is lower than that without autonomous vehicles. And the $75 \%$ and $25 \%$ quantile densities both tend to converge towards the mode density. It shows that the penetration of autonomous vehicles affects for the road traffic density. As the penetration rate exceeds $60 \%$, the density decreases significantly, and the density is further converged. In combination with Figure 7 , it can be seen that as the penetration rate of autonomous vehicles increases, the density presents a nonnormal distribution, and the data is concentrated in the direction of the mode.
The impact of autonomous vehicles on density can be seen in Table 4 and Figure 7. After the autonomous vehicles have penetrated, the density of each lane begins to decrease when the penetration rate reaches more than $60 \%$. Lane 3 and Lane 4 are close to the entrance and exit, especially Lane 4 , which becomes the lane with the largest distance between the $25 \%$ and $75 \%$ quantile in the density distribution. It can be seen that the addition of autonomous vehicles can improve the road density, but this kind of improvement of the whole road section will be highlighted until the penetration rate reaches more than $60 \%$. Otherwise, for multilane roads, the improvement of lanes farther from the entrance and exit will be more obvious. However, the lanes that are close to the entrance or directly connected to the entrance and exit are not significantly improved at the beginning. Autonomous vehicles have a direct impact on lane-changing behavior, as shown in Tables 5 and 6 .

In Table 5, when the penetration rate of self-driving vehicles reaches $10 \%$, the average rate of changing out lanes decreases significantly, and this data increases as the penetration rate increases. It starts to decrease after $40 \%$ of 


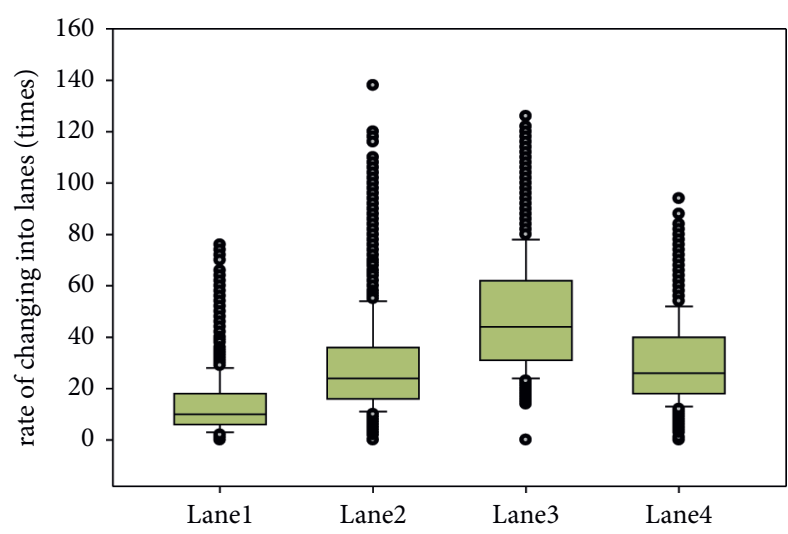

(a)

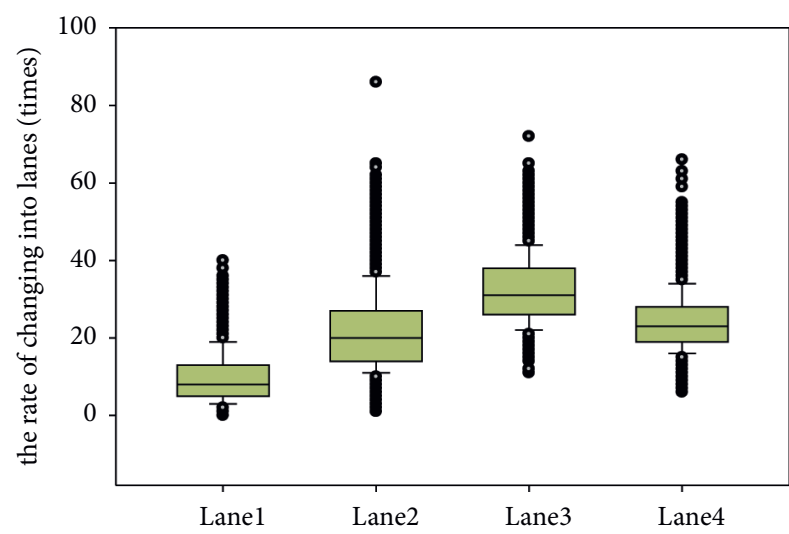

(c)

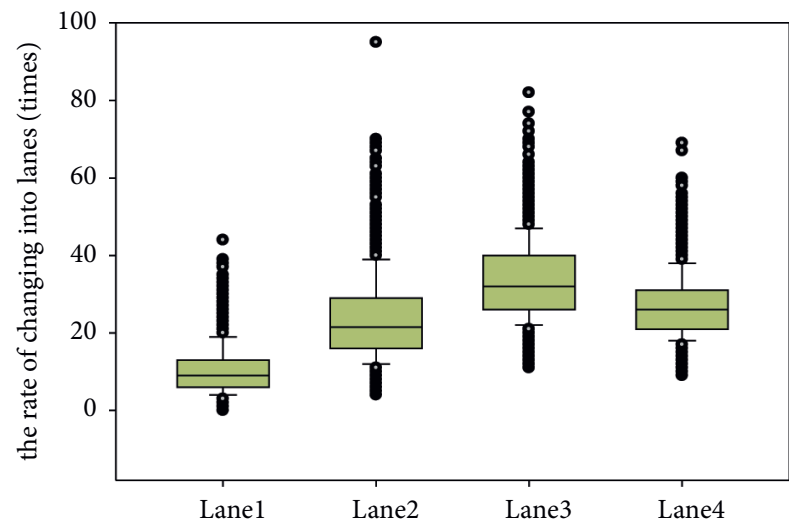

(e)

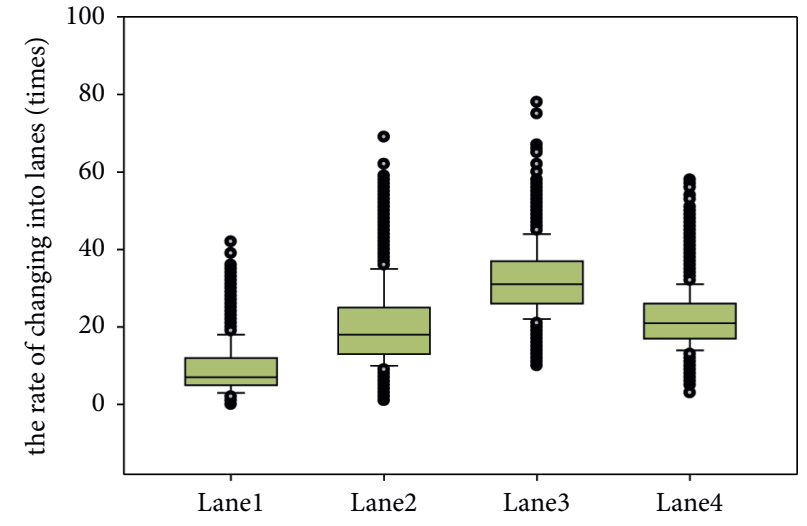

(b)

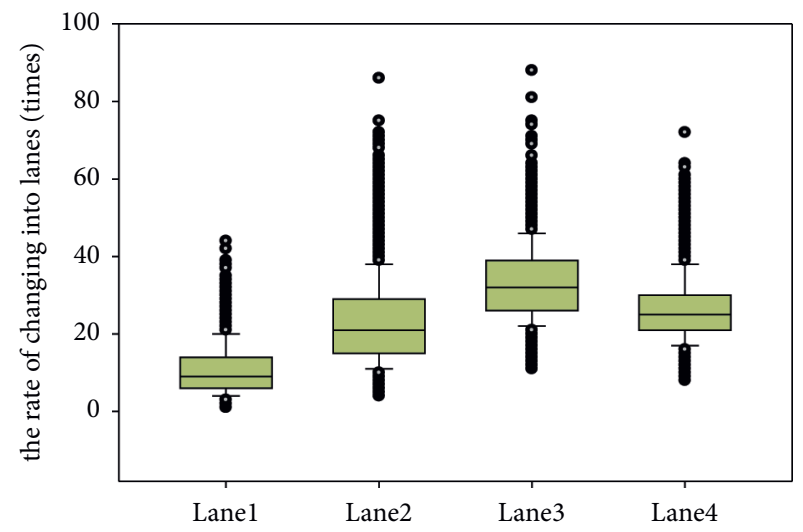

(d)

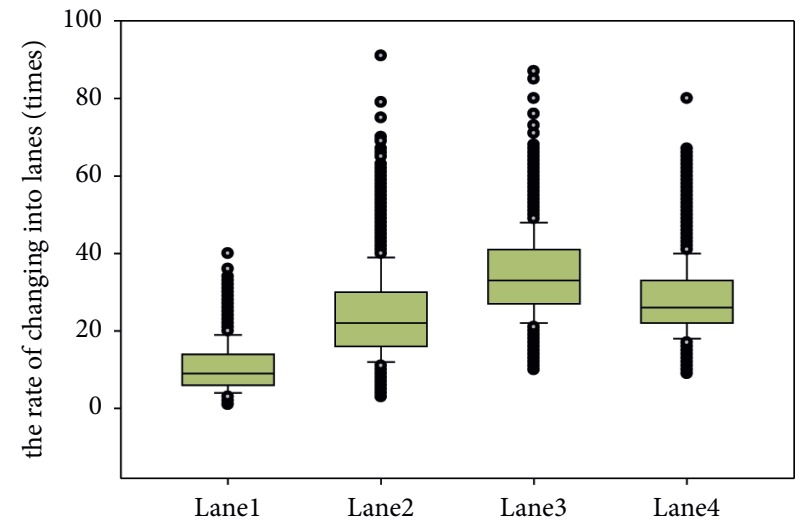

(f)

Figure 9: Continued. 


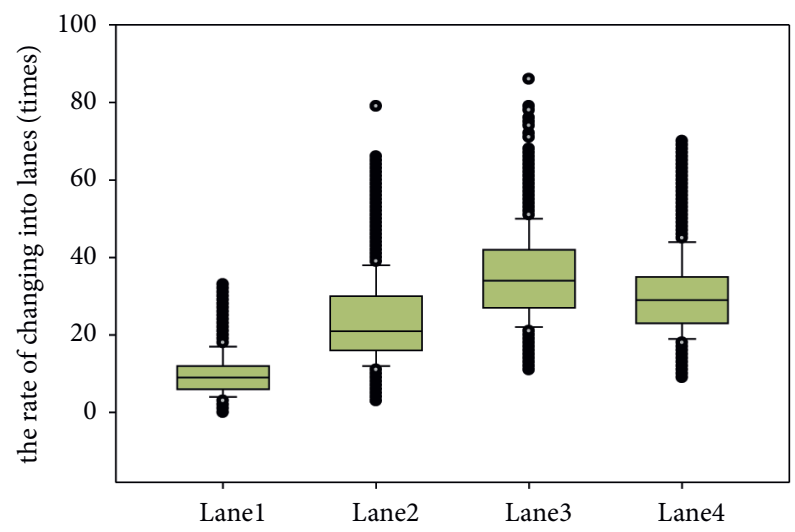

(g)

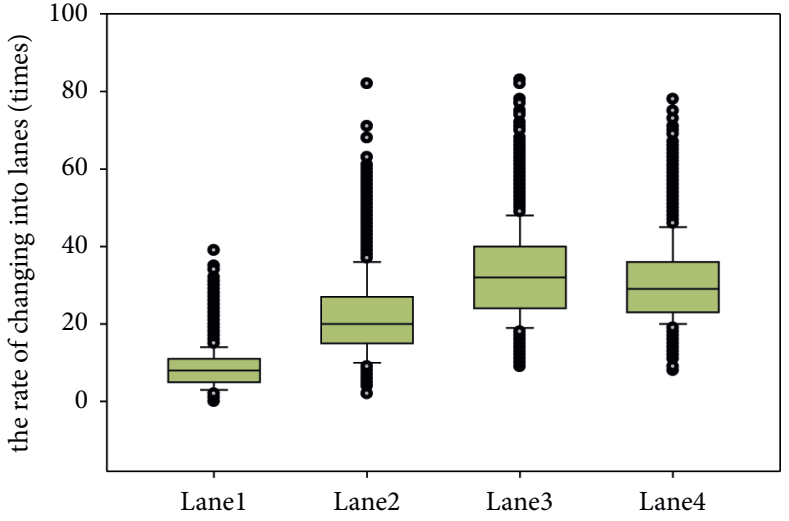

(h)

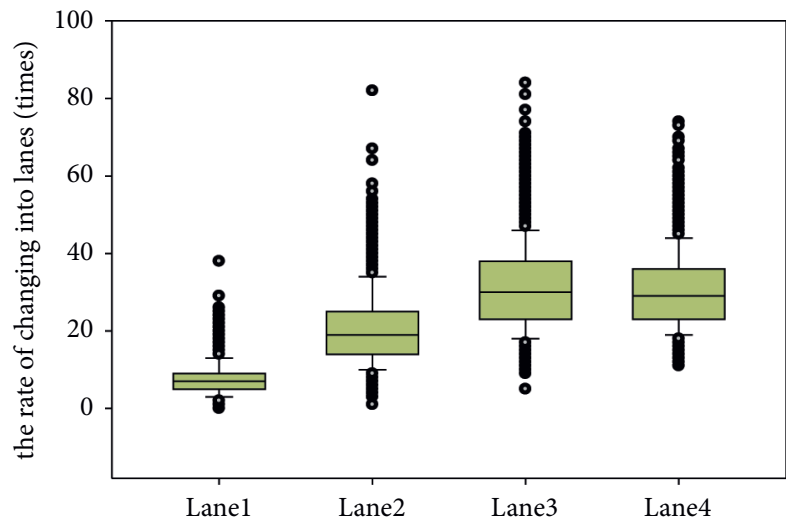

(i)

FIGURE 9: The distribution map of the rate of changing into lanes of a single lane under different autonomous vehicle penetration rates. Distribution map of the rate of changing into lanes (a) for the $0 \%$ joining rate of autonomous vehicles, (b) for the $10 \%$ joining rate of autonomous vehicles, (c) for the $20 \%$ joining rate of autonomous vehicles, (d) for the $30 \%$ joining rate of autonomous vehicles, (e) for the $40 \%$ joining rate of autonomous vehicles, (f) for the $50 \%$ joining rate of autonomous vehicles, (g) for the $60 \%$ joining rate of autonomous vehicles, (h) for the $70 \%$ joining rate of autonomous vehicles, and (i) for the $80 \%$ joining rate of autonomous vehicles.

TABle 4: The density of a single lane under different autonomous vehicle penetration rates by the statistical description (unit: vehicle/km).

\begin{tabular}{ccccccccccc}
\hline & $P=0 \%$ & $P=10 \%$ & $P=20 \%$ & $P=30 \%$ & $P=40 \%$ & $P=50 \%$ & $P=60 \%$ & $P=70 \%$ & $P=80 \%$ \\
\hline \multirow{4}{*}{ Lane 1 } & Mean value & 1.076 & 1.015 & 0.971 & 0.956 & 0.913 & 0.889 & 0.741 & 0.666 & 0.626 \\
& Standard error & 0.007 & 0.007 & 0.006 & 0.007 & 0.006 & 0.006 & 0.004 & 0.003 & 0.003 \\
& Median & 1.110 & 1.030 & 0.970 & 0.930 & 0.895 & 0.865 & 0.730 & 0.660 & 0.625 \\
& Mode & 1.170 & 1.100 & 1.070 & 0.995 & 0.985 & 0.830 & 0.790 & 0.610 & 0.630 \\
& Variance & 0.137 & 0.122 & 0.105 & 0.120 & 0.090 & 0.082 & 0.039 & 0.027 & 0.021 \\
& Kurtosis & 1.165 & 1.854 & 2.864 & 6.652 & 4.397 & 4.144 & 1.574 & 0.883 & 0.020 \\
& Skewness & 0.326 & 0.527 & 0.838 & 1.526 & 1.256 & 1.191 & 0.545 & 0.391 & 0.169 \\
& $75 \%$ & 2.170 & 2.005 & 1.975 & 2.250 & 2.020 & 1.825 & 1.275 & 1.115 & 0.985 \\
& $25 \%$ & 0.350 & 0.335 & 0.350 & 0.345 & 0.355 & 0.375 & 0.330 & 0.310 & 0.310 \\
\hline & Mean value & 5.900 & 5.810 & 5.640 & 5.581 & 5.373 & 5.183 & 4.269 & 3.826 & 3.535 \\
& Standard error & 0.040 & 0.041 & 0.038 & 0.040 & 0.036 & 0.032 & 0.016 & 0.012 & 0.008 \\
& Median & 5.330 & 5.160 & 5.040 & 4.965 & 4.840 & 4.695 & 4.125 & 3.738 & 3.490 \\
& Mode & 5.350 & 5.065 & 4.530 & 4.160 & 4.825 & 4.115 & 3.870 & 3.255 & 3.295 \\
& Variance & 4.144 & 4.416 & 3.777 & 4.224 & 3.388 & 2.698 & 0.693 & 0.354 & 0.169 \\
& Kurtosis & 12.170 & 9.929 & 8.160 & 9.814 & 8.766 & 9.959 & 7.289 & 13.361 & 2.463 \\
& Skewness & 2.921 & 2.771 & 2.514 & 2.677 & 2.572 & 2.538 & 2.063 & 2.264 & 0.941 \\
& $75 \%$ & 15.260 & 15.235 & 13.845 & 13.935 & 12.765 & 11.610 & 7.620 & 6.015 & 4.875 \\
& $25 \%$ & 3.840 & 3.690 & 3.650 & 3.560 & 3.490 & 3.395 & 3.105 & 2.900 & 2.785 \\
\hline
\end{tabular}


TABle 4: Continued.

\begin{tabular}{|c|c|c|c|c|c|c|c|c|c|c|}
\hline & & $P=0 \%$ & $P=10 \%$ & $P=20 \%$ & $P=30 \%$ & $P=40 \%$ & $P=50 \%$ & $P=60 \%$ & $P=70 \%$ & $P=80 \%$ \\
\hline \multirow{9}{*}{ Lane 3} & Mean value & 1.458 & 1.453 & 1.474 & 1.538 & 1.529 & 1.524 & 1.322 & 1.112 & 0.983 \\
\hline & Standard error & 0.013 & 0.013 & 0.013 & 0.013 & 0.012 & 0.012 & 0.011 & 0.009 & 0.007 \\
\hline & Median & 1.320 & 1.295 & 1.318 & 1.395 & 1.400 & 1.400 & 1.215 & 1.015 & 0.920 \\
\hline & Mode & 1.295 & 1.000 & 1.225 & 1.240 & 1.240 & 1.055 & 1.200 & 0.875 & 0.945 \\
\hline & Variance & 0.451 & 0.452 & 0.410 & 0.451 & 0.398 & 0.392 & 0.292 & 0.202 & 0.133 \\
\hline & Kurtosis & 1.090 & 0.780 & 0.598 & 0.654 & 0.939 & 0.708 & 1.793 & 3.493 & 4.681 \\
\hline & Skewness & 0.982 & 0.969 & 0.905 & 0.886 & 0.934 & 0.861 & 1.125 & 1.477 & 1.632 \\
\hline & $75 \%$ & 3.470 & 3.365 & 3.330 & 3.505 & 3.400 & 3.340 & 3.035 & 2.575 & 2.330 \\
\hline & $25 \%$ & 0.400 & 0.350 & 0.395 & 0.415 & 0.500 & 0.500 & 0.435 & 0.425 & 0.420 \\
\hline \multirow{9}{*}{ Lane 4} & Mean value & 8.467 & 8.806 & 9.155 & 9.627 & 9.772 & 9.791 & 9.147 & 8.092 & 7.101 \\
\hline & Standard error & 0.073 & 0.075 & 0.075 & 0.077 & 0.077 & 0.078 & 0.078 & 0.075 & 0.066 \\
\hline & Median & 7.280 & 7.570 & 8.008 & 8.595 & 8.760 & 9.030 & 8.130 & 6.908 & 5.980 \\
\hline & Mode & 4.800 & 5.590 & 7.060 & 5.445 & 7.175 & 6.290 & 5.155 & 4.725 & 4.205 \\
\hline & Variance & 13.731 & 14.607 & 14.404 & 15.424 & 15.219 & 15.569 & 15.728 & 14.718 & 11.444 \\
\hline & Kurtosis & 0.184 & -0.226 & -0.406 & -0.343 & -0.342 & -0.218 & 0.417 & 1.662 & 3.503 \\
\hline & Skewness & 1.001 & 0.861 & 0.742 & 0.711 & 0.700 & 0.658 & 0.957 & 1.324 & 1.693 \\
\hline & $75 \%$ & 18.715 & 18.470 & 18.480 & 19.345 & 19.300 & 19.745 & 20.110 & 20.375 & 18.875 \\
\hline & $25 \%$ & 4.130 & 4.055 & 4.080 & 4.125 & 4.220 & 4.110 & 3.780 & 3.545 & 3.465 \\
\hline
\end{tabular}

TABLE 5: The rate of changing out lanes of a single lane under different autonomous vehicle penetration rates by statistical description (unit: times).

\begin{tabular}{|c|c|c|c|c|c|c|c|c|c|c|}
\hline & & $P=0 \%$ & $P=10 \%$ & $P=20 \%$ & $P=30 \%$ & $P=40 \%$ & $P=50 \%$ & $P=60 \%$ & $P=70 \%$ & $P=80 \%$ \\
\hline \multirow{9}{*}{ Lane 1} & Mean value & 1.076 & 1.015 & 0.971 & 0.956 & 0.913 & 0.889 & 0.741 & 0.666 & 0.626 \\
\hline & Standard error & 0.007 & 0.007 & 0.006 & 0.007 & 0.006 & 0.006 & 0.004 & 0.003 & 0.003 \\
\hline & Median & 1.110 & 1.030 & 0.970 & 0.930 & 0.895 & 0.865 & 0.730 & 0.660 & 0.625 \\
\hline & Mode & 1.170 & 1.100 & 1.070 & 0.995 & 0.985 & 0.830 & 0.790 & 0.610 & 0.630 \\
\hline & Variance & 0.137 & 0.122 & 0.105 & 0.120 & 0.090 & 0.082 & 0.039 & 0.027 & 0.021 \\
\hline & Kurtosis & 1.165 & 1.854 & 2.864 & 6.652 & 4.397 & 4.144 & 1.574 & 0.883 & 0.020 \\
\hline & Skewness & 0.326 & 0.527 & 0.838 & 1.526 & 1.256 & 1.191 & 0.545 & 0.391 & 0.169 \\
\hline & $75 \%$ & 2.170 & 2.005 & 1.975 & 2.250 & 2.020 & 1.825 & 1.275 & 1.115 & 0.985 \\
\hline & $25 \%$ & 0.350 & 0.335 & 0.350 & 0.345 & 0.355 & 0.375 & 0.330 & 0.310 & 0.310 \\
\hline \multirow{9}{*}{ Lane 2} & Mean value & 5.900 & 5.810 & 5.640 & 5.581 & 5.373 & 5.183 & 4.269 & 3.826 & 3.535 \\
\hline & Standard error & 0.040 & 0.041 & 0.038 & 0.040 & 0.036 & 0.032 & 0.016 & 0.012 & 0.008 \\
\hline & Median & 5.330 & 5.160 & 5.040 & 4.965 & 4.840 & 4.695 & 4.125 & 3.738 & 3.490 \\
\hline & Mode & 5.350 & 5.065 & 4.530 & 4.160 & 4.825 & 4.115 & 3.870 & 3.255 & 3.295 \\
\hline & Variance & 4.144 & 4.416 & 3.777 & 4.224 & 3.388 & 2.698 & 0.693 & 0.354 & 0.169 \\
\hline & Kurtosis & 12.170 & 9.929 & 8.160 & 9.814 & 8.766 & 9.959 & 7.289 & 13.361 & 2.463 \\
\hline & Skewness & 2.921 & 2.771 & 2.514 & 2.677 & 2.572 & 2.538 & 2.063 & 2.264 & 0.941 \\
\hline & $75 \%$ & 15.260 & 15.235 & 13.845 & 13.935 & 12.765 & 11.610 & 7.620 & 6.015 & 4.875 \\
\hline & $25 \%$ & 3.840 & 3.690 & 3.650 & 3.560 & 3.490 & 3.395 & 3.105 & 2.900 & 2.785 \\
\hline \multirow{9}{*}{ Lane 3} & Mean value & 1.458 & 1.453 & 1.474 & 1.538 & 1.529 & 1.524 & 1.322 & 1.112 & 0.983 \\
\hline & Standard error & 0.013 & 0.013 & 0.013 & 0.013 & 0.012 & 0.012 & 0.011 & 0.009 & 0.007 \\
\hline & Median & 1.320 & 1.295 & 1.318 & 1.395 & 1.400 & 1.400 & 1.215 & 1.015 & 0.920 \\
\hline & Mode & 1.295 & 1.000 & 1.225 & 1.240 & 1.240 & 1.055 & 1.200 & 0.875 & 0.945 \\
\hline & Variance & 0.451 & 0.452 & 0.410 & 0.451 & 0.398 & 0.392 & 0.292 & 0.202 & 0.133 \\
\hline & Kurtosis & 1.090 & 0.780 & 0.598 & 0.654 & 0.939 & 0.708 & 1.793 & 3.493 & 4.681 \\
\hline & Skewness & 0.982 & 0.969 & 0.905 & 0.886 & 0.934 & 0.861 & 1.125 & 1.477 & 1.632 \\
\hline & $75 \%$ & 3.470 & 3.365 & 3.330 & 3.505 & 3.400 & 3.340 & 3.035 & 2.575 & 2.330 \\
\hline & $25 \%$ & 0.400 & 0.350 & 0.395 & 0.415 & 0.500 & 0.500 & 0.435 & 0.425 & 0.420 \\
\hline \multirow{9}{*}{ Lane 4} & Mean value & 8.467 & 8.806 & 9.155 & 9.627 & 9.772 & 9.791 & 9.147 & 8.092 & 7.101 \\
\hline & Standard error & 0.073 & 0.075 & 0.075 & 0.077 & 0.077 & 0.078 & 0.078 & 0.075 & 0.066 \\
\hline & Median & 7.280 & 7.570 & 8.008 & 8.595 & 8.760 & 9.030 & 8.130 & 6.908 & 5.980 \\
\hline & Mode & 4.800 & 5.590 & 7.060 & 5.445 & 7.175 & 6.290 & 5.155 & 4.725 & 4.205 \\
\hline & Variance & 13.731 & 14.607 & 14.404 & 15.424 & 15.219 & 15.569 & 15.728 & 14.718 & 11.444 \\
\hline & Kurtosis & 0.184 & -0.226 & -0.406 & -0.343 & -0.342 & -0.218 & 0.417 & 1.662 & 3.503 \\
\hline & Skewness & 1.001 & 0.861 & 0.742 & 0.711 & 0.700 & 0.658 & 0.957 & 1.324 & 1.693 \\
\hline & $75 \%$ & 18.715 & 18.470 & 18.480 & 19.345 & 19.300 & 19.745 & 20.110 & 20.375 & 18.875 \\
\hline & $25 \%$ & 4.130 & 4.055 & 4.080 & 4.125 & 4.220 & 4.110 & 3.780 & 3.545 & 3.465 \\
\hline
\end{tabular}


TABLE 6: The rate of changing into lanes of a single lane under different autonomous vehicle penetration rates by statistical description (unit: times).

\begin{tabular}{|c|c|c|c|c|c|c|c|c|c|c|}
\hline & & $P=0 \%$ & $P=10 \%$ & $P=20 \%$ & $P=30 \%$ & $P=40 \%$ & $P=50 \%$ & $P=60 \%$ & $P=70 \%$ & $P=80 \%$ \\
\hline \multirow{9}{*}{ Lane 1} & Mean value & 11.761 & 8.075 & 8.151 & 8.318 & 8.174 & 8.084 & 6.857 & 5.926 & 5.500 \\
\hline & Standard error & 0.159 & 0.087 & 0.083 & 0.082 & 0.076 & 0.076 & 0.067 & 0.059 & 0.056 \\
\hline & Median & 10.000 & 8.000 & 8.000 & 8.000 & 8.000 & 8.000 & 6.000 & 6.000 & 5.000 \\
\hline & Mode & 8.000 & 6.000 & 6.000 & 6.000 & 6.000 & 6.000 & 5.000 & 5.000 & 4.000 \\
\hline & Variance & 65.107 & 19.75 & 17.88 & 17.50 & 15.04 & 15.028 & 11.612 & 9.024 & 8.242 \\
\hline & Kurtosis & 1.640 & 0.829 & 0.842 & 1.221 & 1.084 & 0.953 & 1.072 & 1.732 & 1.382 \\
\hline & Skewness & 1.180 & 0.845 & 0.801 & 0.963 & 0.839 & 0.889 & 0.805 & 0.966 & 0.899 \\
\hline & $75 \%$ & 38.000 & 21.00 & 20.00 & 22.00 & 20.00 & 20.000 & 16.000 & 15.000 & 14.000 \\
\hline & $25 \%$ & 0.000 & 1.000 & 1.000 & 2.000 & 1.000 & 2.000 & 1.000 & 1.000 & 1.000 \\
\hline \multirow{9}{*}{ Lane 2} & Mean value & 23.350 & 16.11 & 16.73 & 17.64 & 17.65 & 17.983 & 17.254 & 16.065 & 15.286 \\
\hline & Standard error & 0.280 & 0.161 & 0.162 & 0.181 & 0.166 & 0.174 & 0.167 & 0.157 & 0.143 \\
\hline & Median & 20.000 & 14.00 & 15.00 & 16.00 & 16.00 & 16.000 & 15.000 & 15.000 & 14.000 \\
\hline & Mode & 18.000 & 14.00 & 13.00 & 12.00 & 14.00 & 13.000 & 12.000 & 11.000 & 11.000 \\
\hline & Variance & 203.59 & 66.69 & 68.34 & 84.86 & 71.45 & 78.740 & 71.974 & 63.963 & 53.225 \\
\hline & Kurtosis & 2.949 & 2.163 & 1.877 & 3.032 & 2.510 & 2.126 & 1.594 & 2.658 & 2.387 \\
\hline & Skewness & 1.462 & 1.335 & 1.239 & 1.445 & 1.311 & 1.275 & 1.145 & 1.323 & 1.243 \\
\hline & $75 \%$ & 70.000 & 44.00 & 44.00 & 49.00 & 46.00 & 47.000 & 44.000 & 43.000 & 39.000 \\
\hline & $25 \%$ & 4.000 & 4.000 & 4.000 & 5.000 & 5.000 & 4.000 & 4.000 & 4.000 & 3.000 \\
\hline \multirow{9}{*}{ Lane 3} & Mean value & 47.301 & 34.71 & 37.64 & 41.20 & 42.63 & 44.017 & 47.123 & 46.893 & 45.844 \\
\hline & Standard error & 0.458 & 0.241 & 0.260 & 0.298 & 0.291 & 0.309 & 0.335 & 0.339 & 0.316 \\
\hline & Median & 42.000 & 32.00 & 35.00 & 38.00 & 40.00 & 41.000 & 44.000 & 43.000 & 43.000 \\
\hline & Mode & 36.000 & 30.00 & 33.00 & 32.00 & 32.00 & 37.000 & 42.000 & 36.000 & 41.000 \\
\hline & Variance & 543.72 & 150.6 & 175.2 & 230.7 & 218.8 & 247.65 & 290.65 & 297.918 & 259.274 \\
\hline & Kurtosis & 1.679 & 2.861 & 2.250 & 2.248 & 1.934 & 1.732 & 0.814 & 1.004 & 1.160 \\
\hline & Skewness & 1.129 & 1.481 & 1.344 & 1.310 & 1.210 & 1.108 & 0.946 & 0.986 & 0.975 \\
\hline & $75 \%$ & 126.000 & 77.000 & 83.000 & 89.000 & 91.000 & 93.000 & 98.000 & 99.000 & 94.000 \\
\hline & $25 \%$ & 14.000 & 16.000 & 17.000 & 18.000 & 19.000 & 19.000 & 20.000 & 20.000 & 20.000 \\
\hline \multirow{9}{*}{ Lane 4} & Mean value & 38.018 & 25.357 & 25.288 & 26.016 & 26.571 & 26.881 & 27.165 & 25.524 & 23.642 \\
\hline & Standard error & 0.343 & 0.149 & 0.148 & 0.152 & 0.164 & 0.165 & 0.174 & 0.180 & 0.173 \\
\hline & Median & 34.000 & 24.000 & 24.000 & 25.000 & 26.000 & 26.000 & 26.000 & 25.000 & 23.000 \\
\hline & Mode & 17.442 & 7.574 & 7.548 & 7.738 & 8.341 & 8.377 & 8.843 & 9.171 & 8.813 \\
\hline & Variance & 304.219 & 57.369 & 56.978 & 59.879 & 69.569 & 70.169 & 78.200 & 84.110 & 77.671 \\
\hline & Kurtosis & 0.115 & 1.349 & -0.052 & -0.118 & 0.273 & -0.053 & 0.010 & 0.173 & -0.001 \\
\hline & Skewness & 0.798 & 0.683 & 0.497 & 0.449 & 0.581 & 0.507 & 0.528 & 0.562 & 0.534 \\
\hline & $75 \%$ & 86.000 & 46.000 & 45.000 & 46.000 & 49.000 & 49.000 & 51.000 & 51.000 & 46.000 \\
\hline & $25 \%$ & 12.000 & 11.000 & 11.000 & 11.000 & 11.000 & 11.000 & 11.000 & 9.000 & 8.000 \\
\hline
\end{tabular}

Lane 1. Lane 2 did not begin to decline until 60\%, and the data for Lane 3 and Lane 4 did not show a downward trend until the penetration rate reached $70 \%$. Although when the penetration rate reaches $80 \%$, the average rate of changing out lanes decreases compared with the case of no autonomous vehicle, but in $10 \%$ case of the penetration rate of autonomous vehicles, lane-changing behavior of all lanes has an obvious decrease, as the penetration rate increases, the lane-changing behavior will increase, and the changes in each lane are also different. The median and mode of lane swapping rates show a downward trend in Lane 1, Lane 2, and Lane 4, while Lane 3 is not obvious. The 75\% quantile of the lane swap rate showed a downward trend in Lane 1 to Lane 4 , while the $25 \%$ quantile showed an upward trend in Lane 3, Lane 4 showed a downward trend, and Lane 1 and Lane 2 were not obvious. The final data converges towards the mode. The situation in Table 6 is similar to that in Table 5, except that the average rate of changing into lanes in Lane 4 decreases sharply with the addition of autonomous vehicles and then increases and is finally close to the situation without autonomous vehicles. The data all show a tendency to converge in the direction of the mode. The performance of the remaining data is relatively close. Combining Figures 8, 9, Tables 5, and 6, it can be seen that, with the addition of autonomous vehicles, it has an impact on lane-changing behavior. However, the rate of changing into lanes and the rate of changing out lanes are both declined or remained with no autonomous vehicle when the penetration rate reaches $80 \%$, but as the penetration increased, the lane change rate showed a law of first decreasing and then slightly increasing, reaching a certain percentage and then decreasing. Due to the inconsistency distance between the ramps and different lanes, different lanes have different rate of changing into lanes and rate of changing out lanes. However, Lane 3 , as the busiest lane on the entire road, burden on a large number of lane changes. 
TABLE 7: The linear correlation of $k$ and LCi, and LCo.

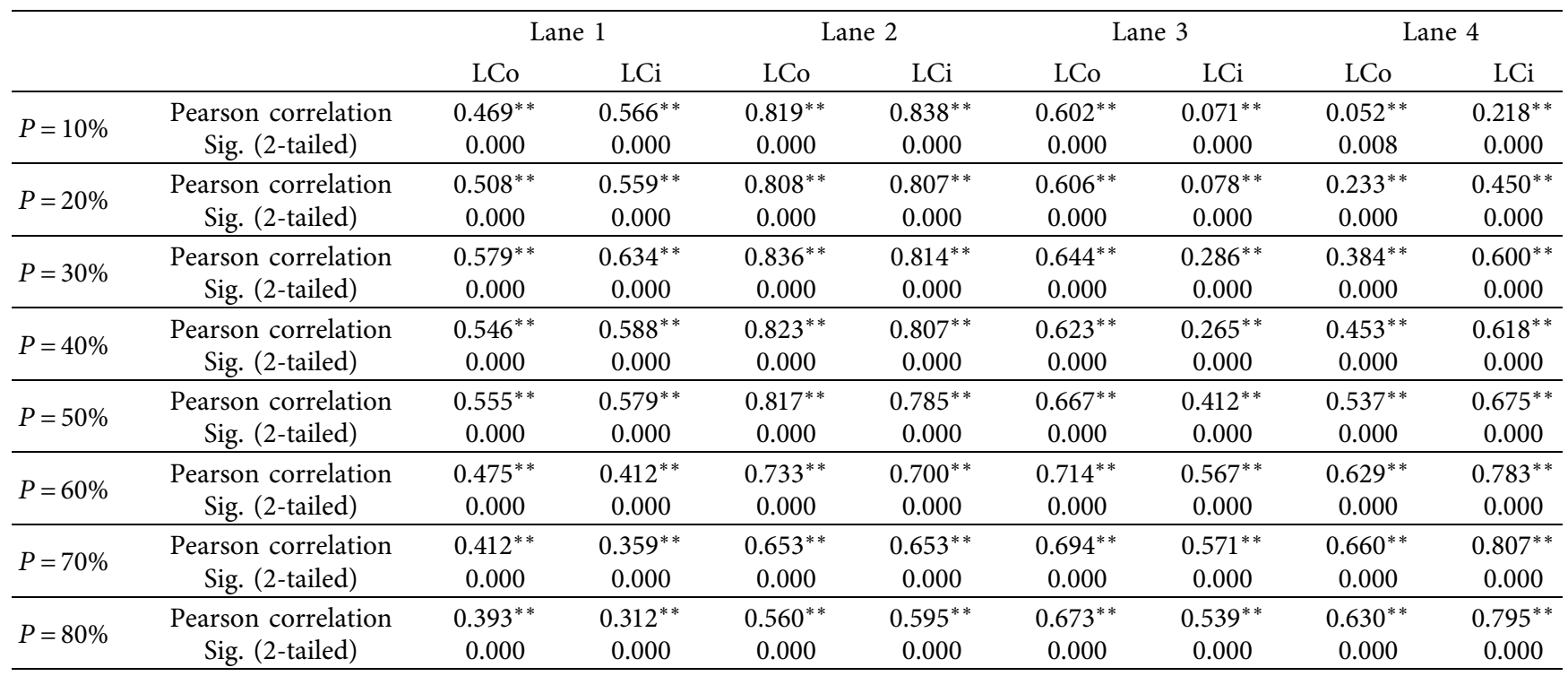

Note: (a) * when 0.05 , significant correlation. (b) ${ }^{* *}$ when 0.01 , significant correlation.

The lane-changing behavior of Lane 4 does not respond significantly with the addition of autonomous vehicles. However, all data show that as the penetration rate of autonomous vehicles increases, there is a certain degree of convergence in lane density, the rate of changing into lanes, and the rate of changing out lanes. Through the above analysis, we believe that the relationship between density and lane-changing behavior requires further research and analysis.

\section{Analysis and Comparison}

4.1. Analysis of the Relationship between Density and LaneChanging Behavior in Mixed Traffic. From the previous analysis, we can see that there is a certain relationship between density and lane-changing behavior. In terms of the form of data, with the penetration of autonomous vehicles, the density and lane-changing behavior have improved. For the relationship between density and lane-changing behavior under different permeability, different lanes need to be analyzed accordingly.

In order to verify whether there is a linear correlation between the density and the lane change rate, the correlation analysis between the density and the lane change rate in each lane under the different penetration rates of autonomous vehicles is shown in Table 7.

It can be seen from Table 7 that, with the increase in the penetration rate of autonomous vehicles, the correlation between the density and the lane change-out rate and lane change-in rate in Lane 1 increased before the penetration rate reached $30 \%$ and then continued to decrease. Lane 2 decreased as the penetration rate increased after $30 \%$. Lane 3 and Lane 4 have increased and decreased, and the relationship change is not obvious. The relationship between density and the rate of changing into lanes, and density and the rate of changing out lanes can be observed more accurately from the scatter plot of the relationship between them, as shown in Figures 10 and 11.

It can be seen from Figures 10 and 11 that, with the penetration of autonomous vehicles, the relationship between density and the rate of changing into lanes and the relationship between density and the rate of changing out lanes appears basically the same in the same lane, but displays in different lanes, the shapes are slightly different. However, they gather in the direction of lower density and slightly fewer lanes. In Figure 10, as the penetration rate of autonomous vehicles increases, the rate of lane swapping out increases, and the degree of dispersion decreases. Especially when the permeability increases to more than $50 \%$, this situation is more obvious. In Figure 11, although the aggregation also shows an increasing trend, the performance is not obvious in Lane 2. In Figure 10, the density in Lane 2 and Lane 3 and the lane change rate show a relatively obvious linear relationship, while the relationship between Lane 1 and Lane 4 is not obvious. The same linear relationship is shown in the graph of the relationship in Lane 2 and Lane 4 after the penetration rate over $50 \%$ in Figure 11. In Lane 1 and Lane 3, the appearance of this relationship is not obvious. However, from Lane 1 and Lane 4 in Figure 10, and Lane 1 and Lane 3 in Figure 11, it can also be seen that the data has a tendency to converge and develop linearly. Therefore, the possibility of a linear relationship between density and lane-changing behavior can be considered. Multiple regression is used to describe the relationship between them.

4.2. Density-Lane-Changing Behavior Regression Model. It can be seen from Figures 10, 11, and Table 7 that there is a significant linear relationship between the density and the rate of changing into lanes, and the density and the rate of changing out lanes. Multiple regression is used to describe it. 

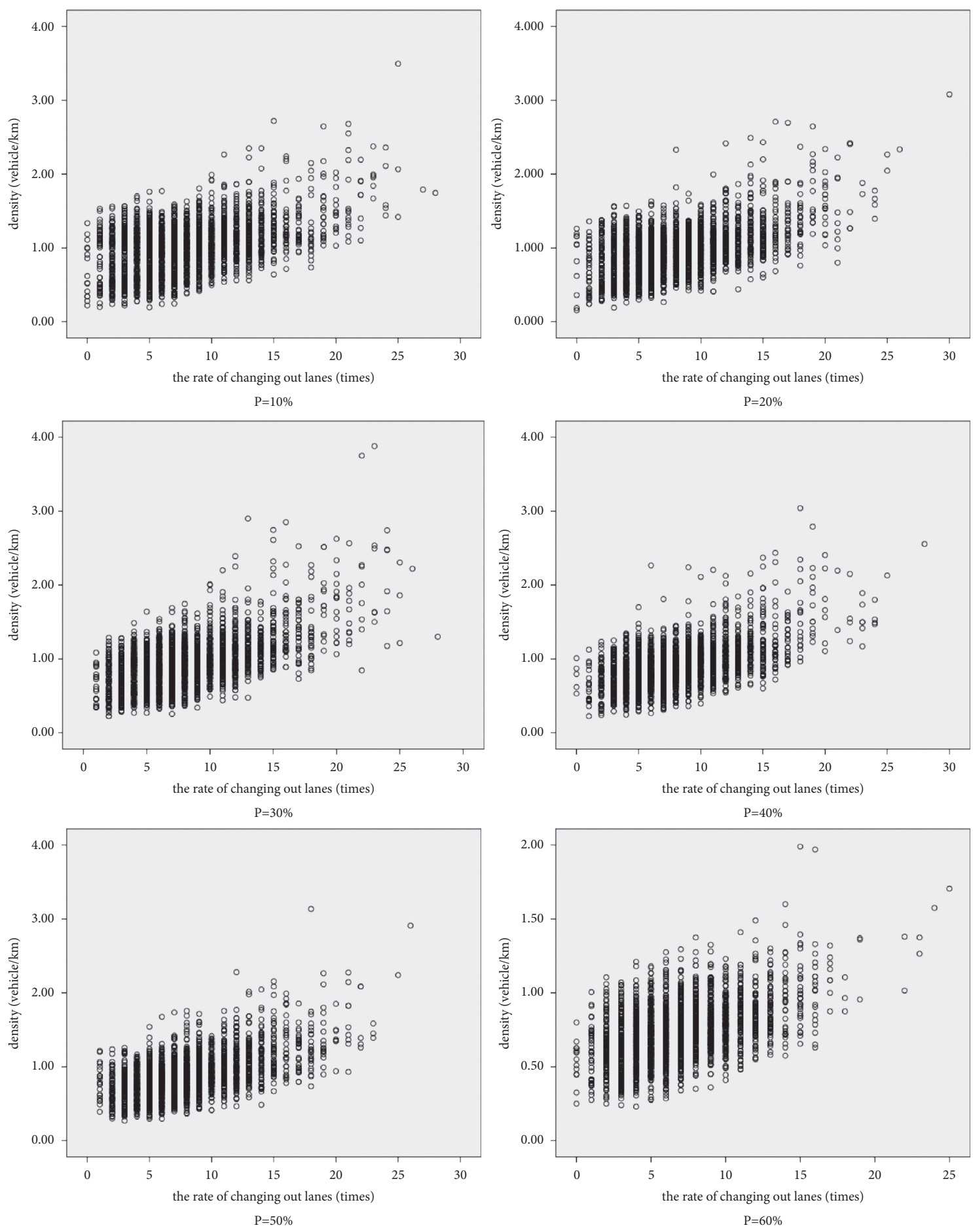

FIgURE 10(a): Continued. 

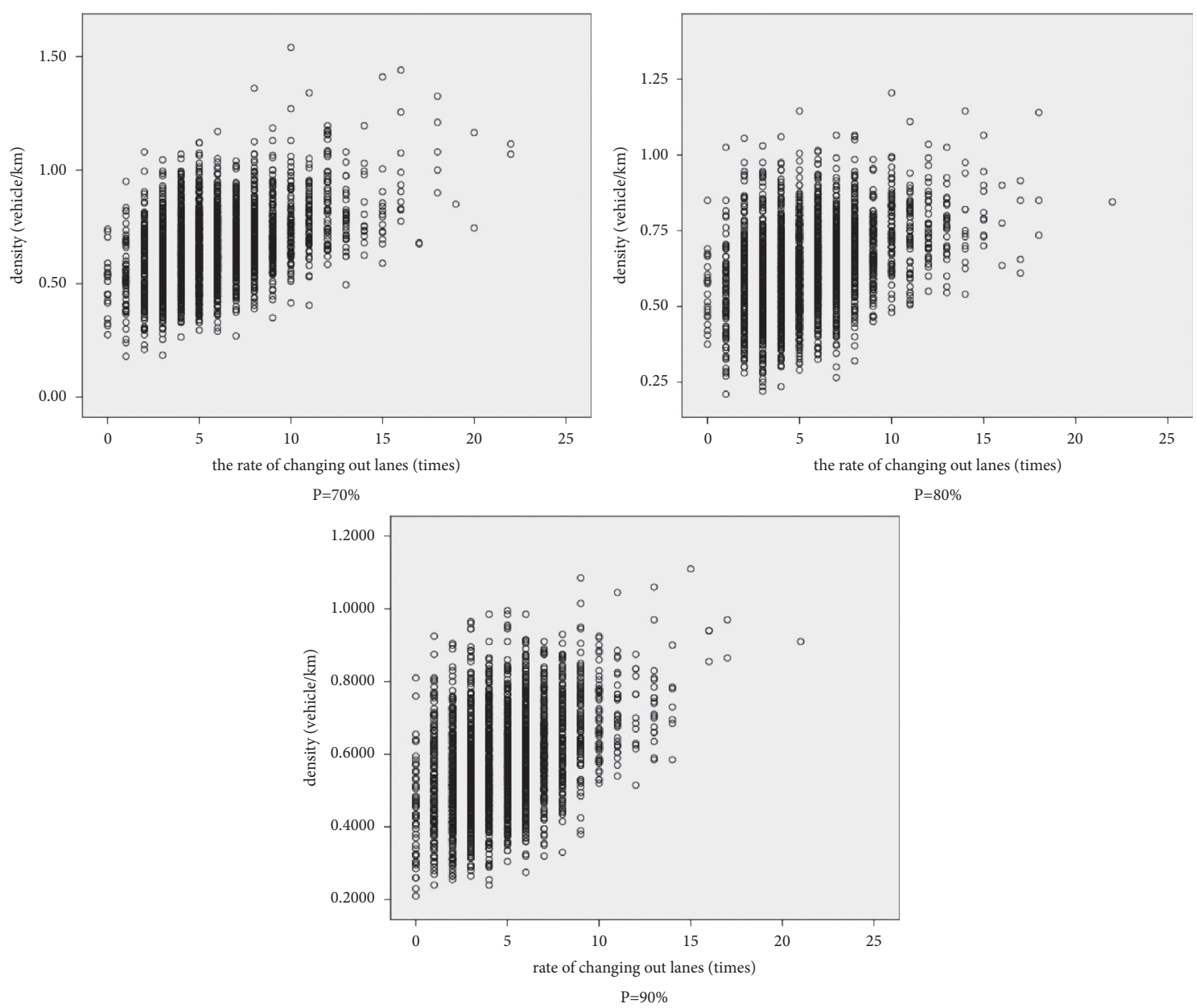

(a)

Figure 10: Continued. 

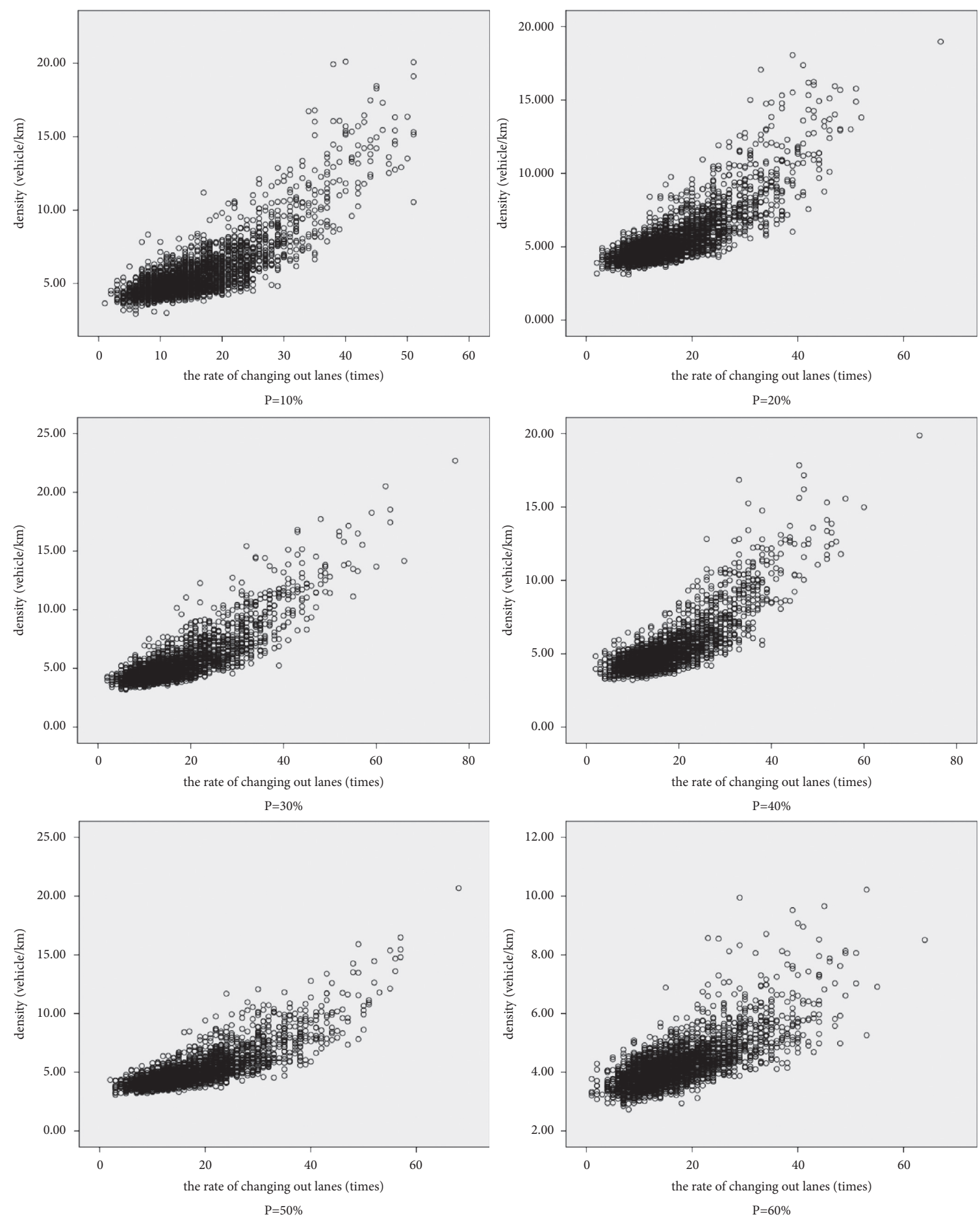

FIgURE 10(b): Continued. 

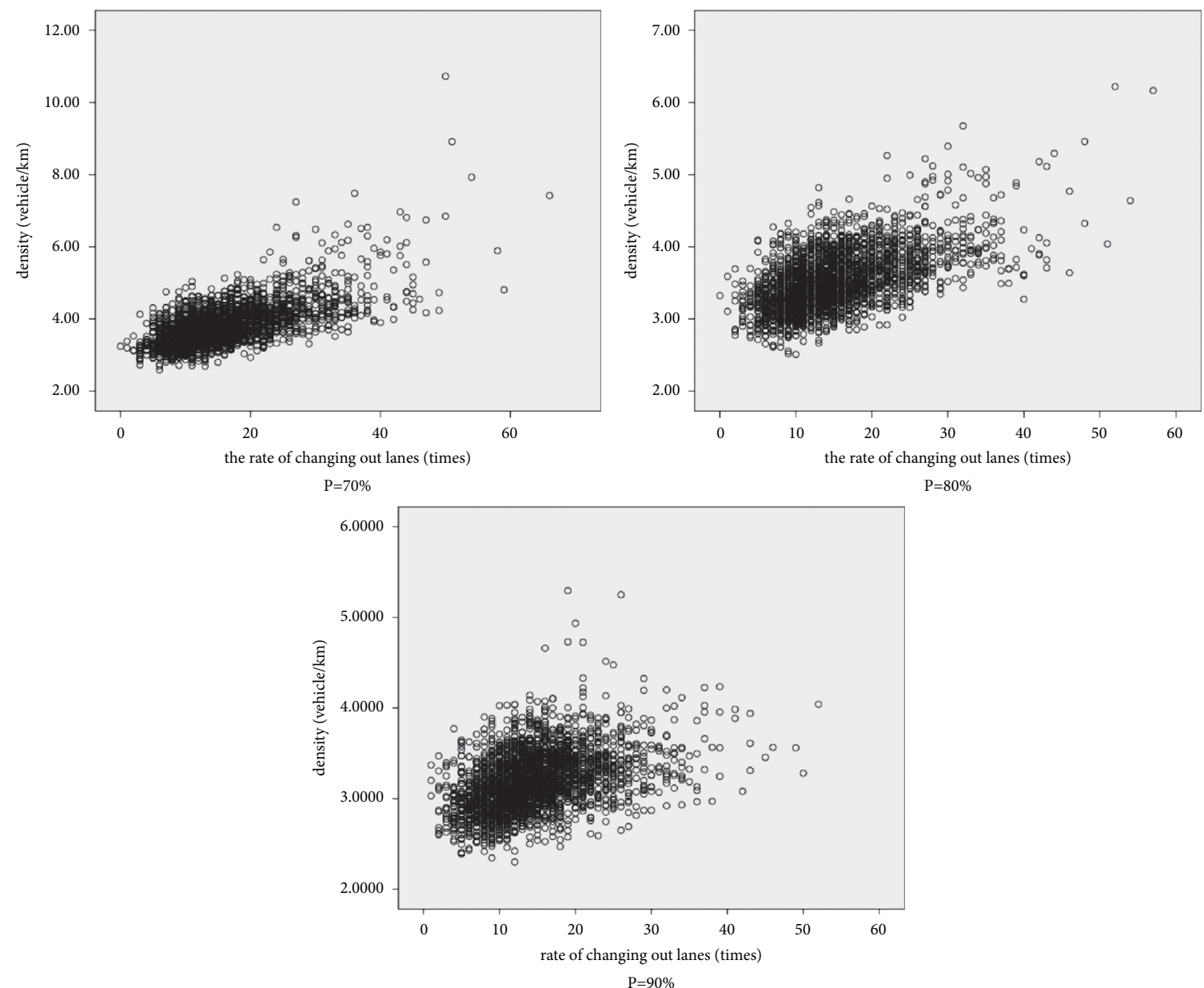

(b)

Figure 10: Continued. 

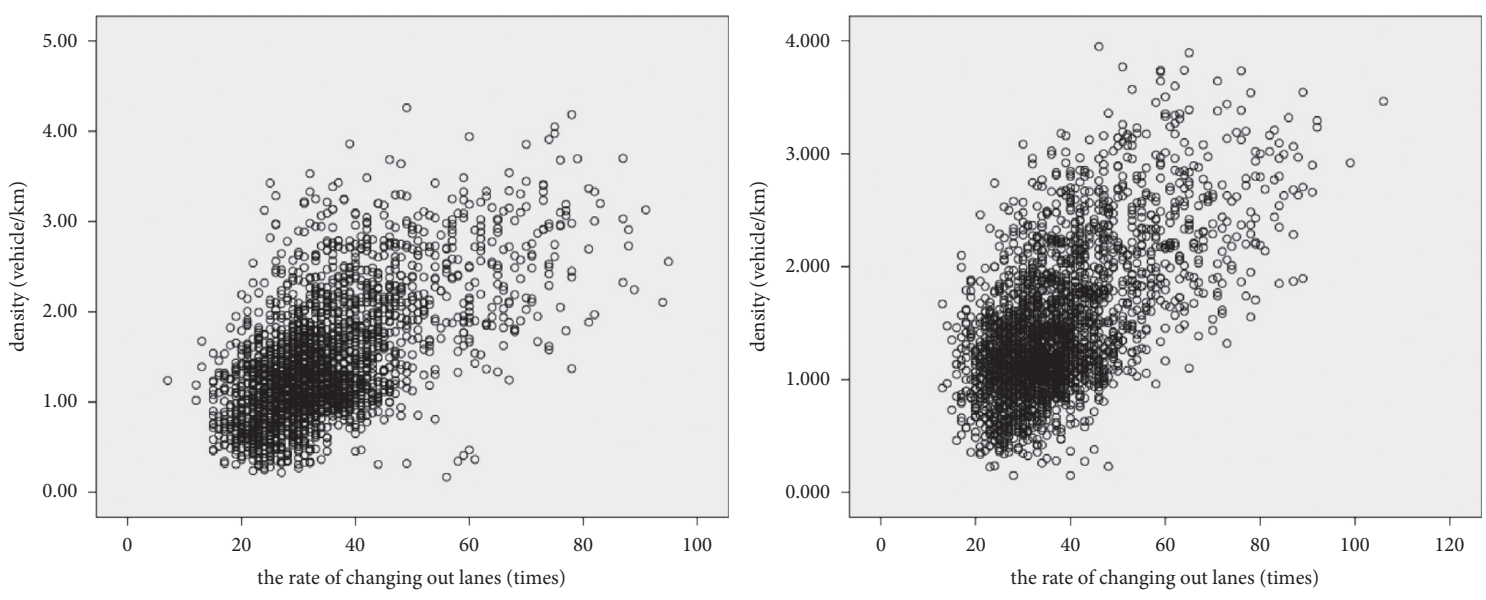

$\mathrm{P}=10 \%$
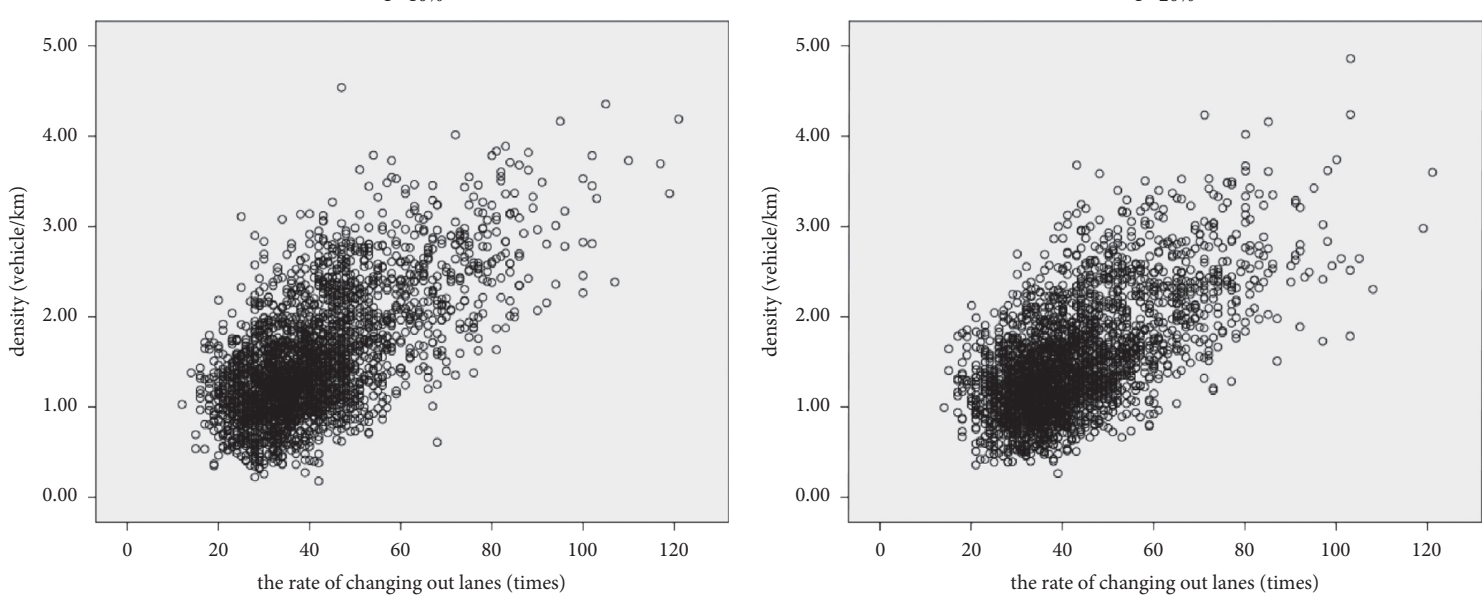

$\mathrm{P}=30 \%$

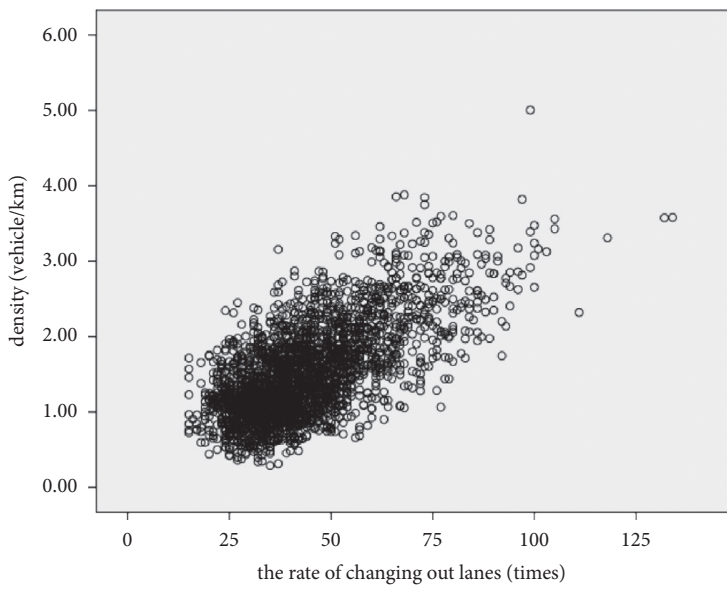

$\mathrm{P}=50 \%$

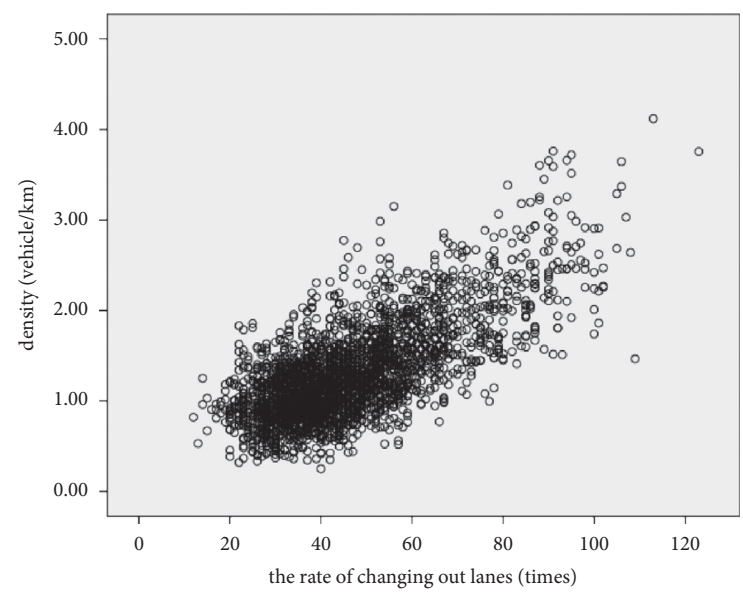

$\mathrm{P}=60 \%$

Figure 10 (c): Continued. 

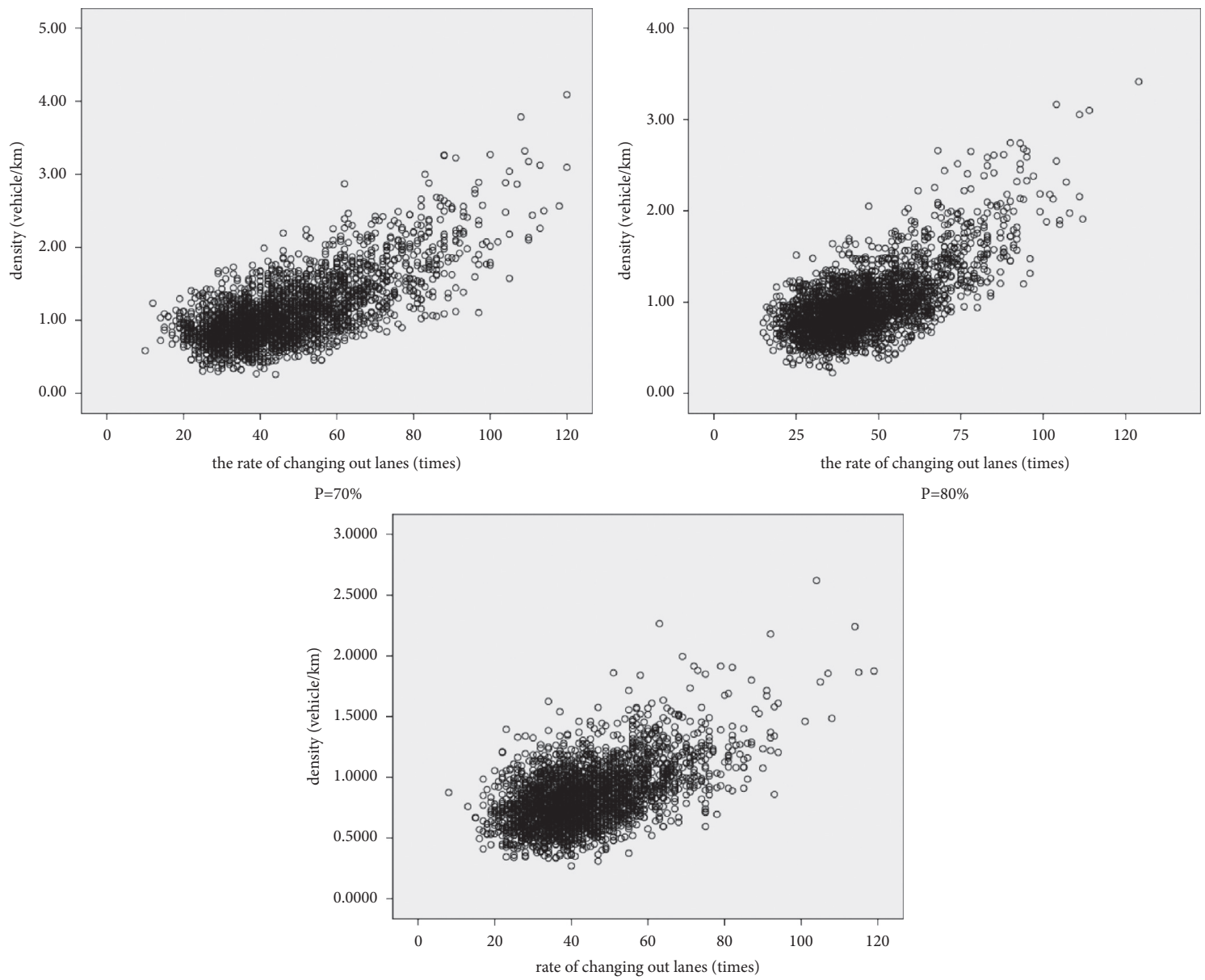

$\mathrm{P}=90 \%$

(c)

Figure 10: Continued. 

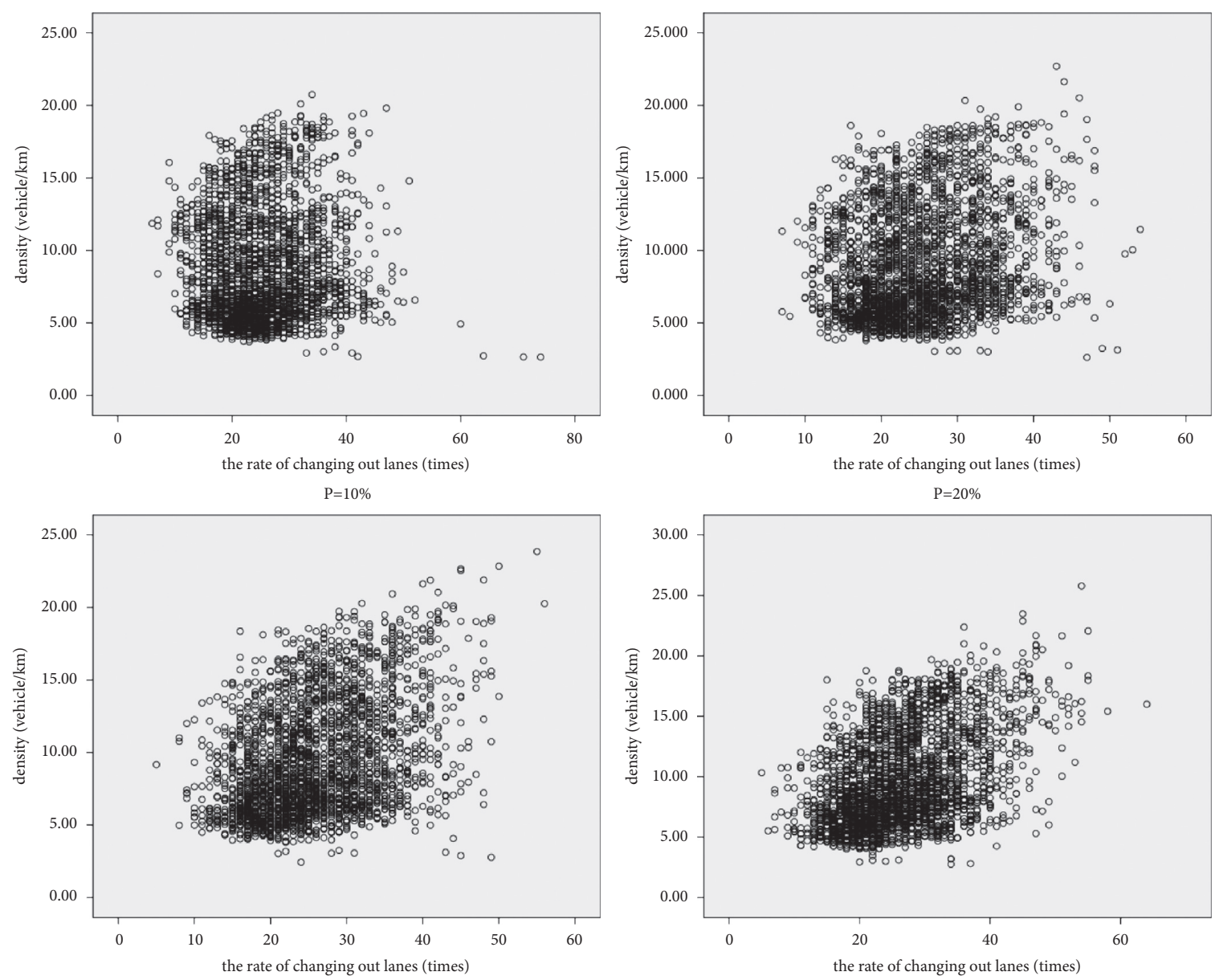

$\mathrm{P}=30 \%$
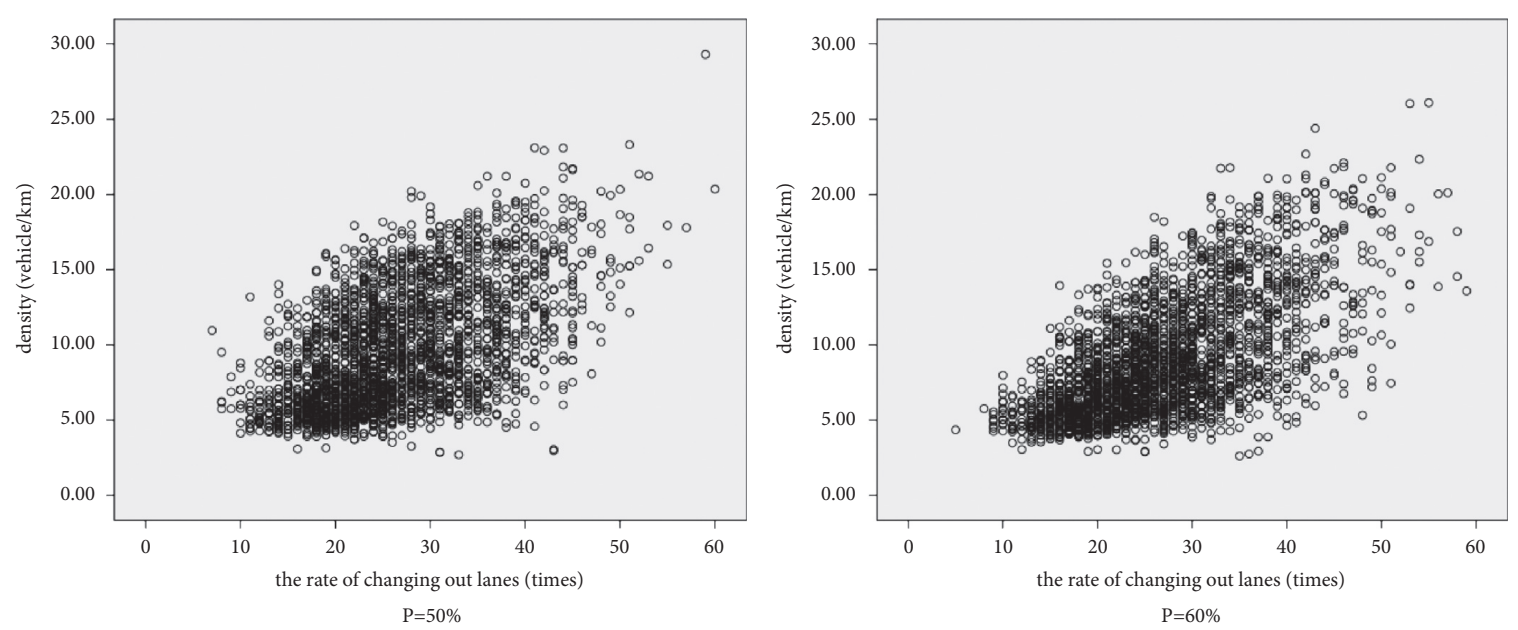

FIGURE 10(d): Continued. 

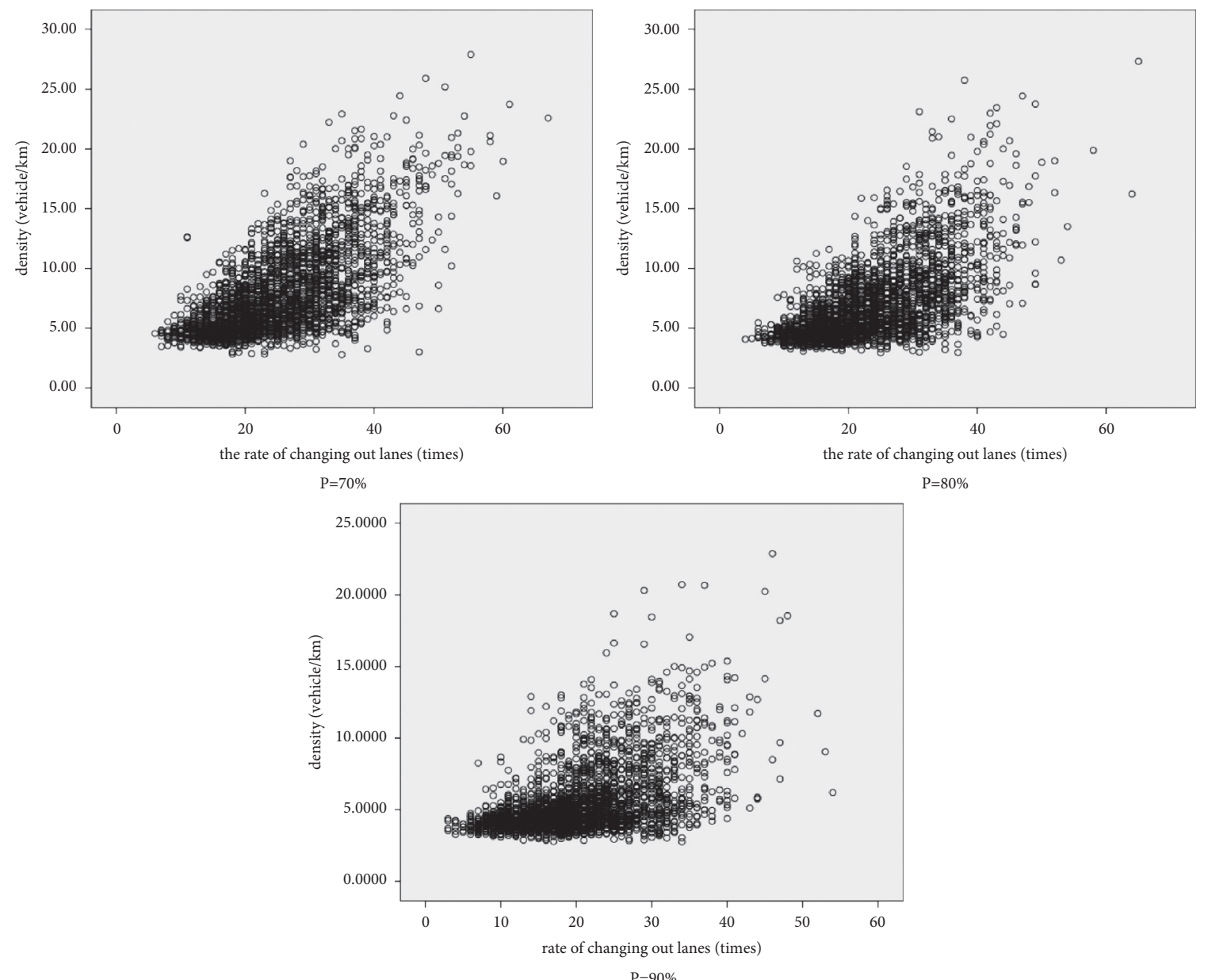

$\mathrm{P}=80 \%$

(d)

Figure 10: The relationship between density and the rate of changing out lanes by scatter plot. (a) The scatter plot of Lane 1. (b) The scatter plot of Lane 2. (c) The scatter plot of Lane 3. (d) The scatter plot of Lane 4.

Assume that the relationship between the density $k$ and the rate of changing out lanes LCo and the rate of changing into lanes $\mathrm{LCi}$ is expressed by

$$
k=f(L C o, L C i) .
$$

Assume that the relationship between the density $k$ and the rate of changing out lanes LCo and the rate of changing into lanes LCi is shown in

$$
k=a L C o+b L C i+c,
$$

where a, $b$, and $c$ all represent constants.

The relationship between the density and lane-changing behavior under different autonomous vehicle penetration rates was analyzed by multiple regression analysis, and the fit degree is shown in Table 8.

It can be seen from Table 8 that the degree of fit $\mathrm{R}$ in Lane 1 increases as the penetration rate increases to $50 \%$ and then shows a downward trend. The same trend is observed in Lane 2, but the penetration rate in Lane 2 shows a downward trend after $30 \%$, but it is not obvious, and it does not decrease significantly until after $60 \%$. Lane 3 does not have this trend. Lane 4 does not increase significantly with the penetration of autonomous vehicles. This trend slows down when the penetration rate reaches $30 \%$, and there is a more obvious increase after $50 \%$, and after $60 \%$, it slows down. However, from the perspective of the degree of fit, with the penetration of autonomous vehicles, there is a certain linear relationship between the density and the rate of changing into lanes and the rate of changing out lanes, although the penetration rate on Lane 1 reaches $80 \%$. The correlation is low, and Lane 4 has a low correlation when the penetration rate is $10 \%$. It shows that when the autonomous vehicles begin to penetrate, the relationship between lane-changing behavior and density is not obvious because Lane 4 is directly connected to the entrance and exit. Because there are fewer autonomous vehicles, this relationship does not appear. When the number of autonomous vehicles continues to increase, the influence on the relationship between density and lane-changing behavior presents a more obvious linear 

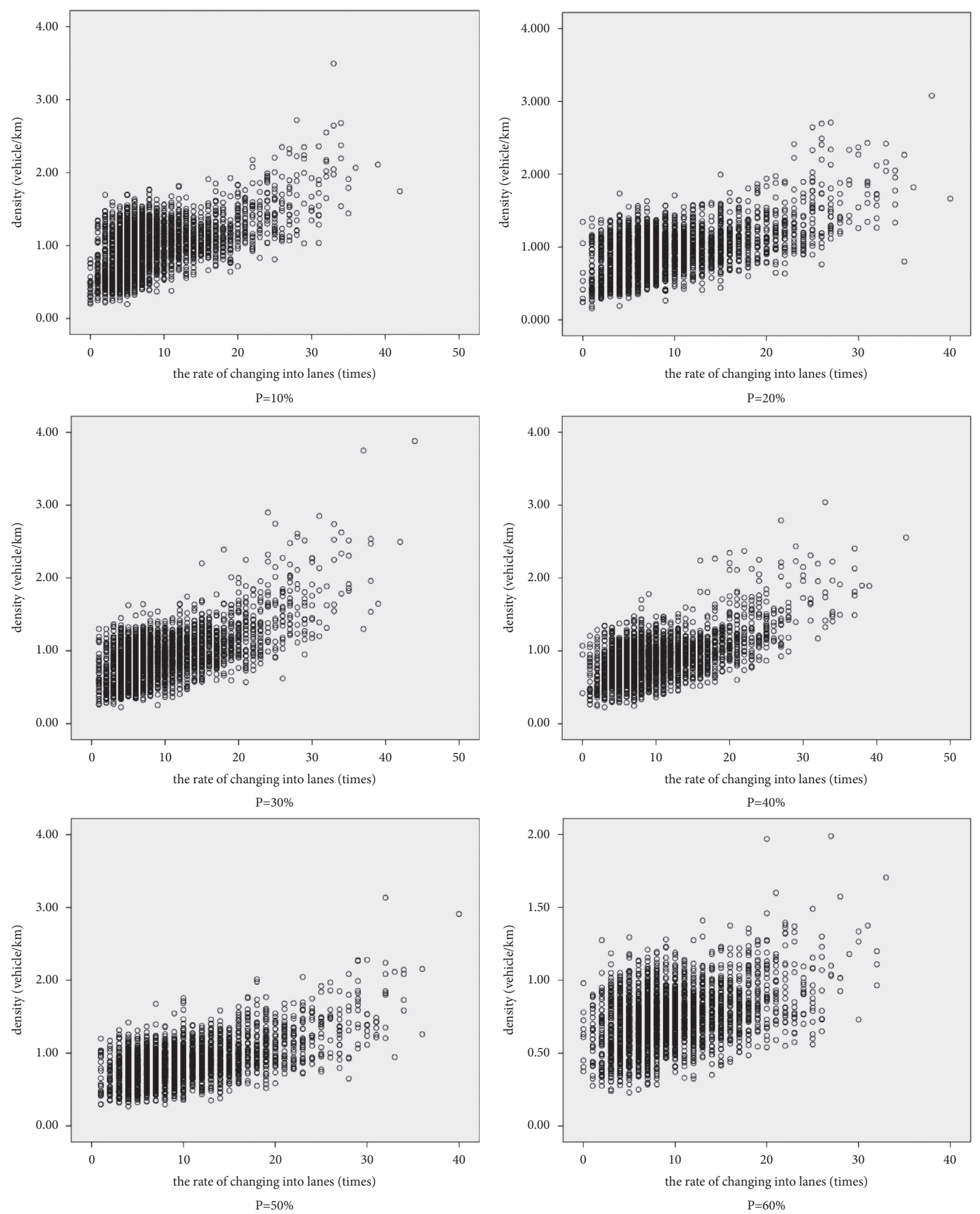

Figure 11(a): Continued. 


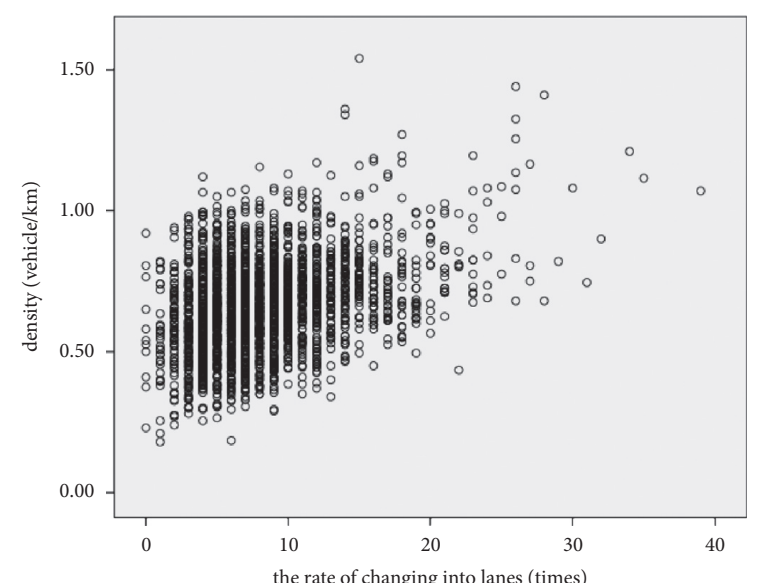

$\mathrm{P}=70 \%$
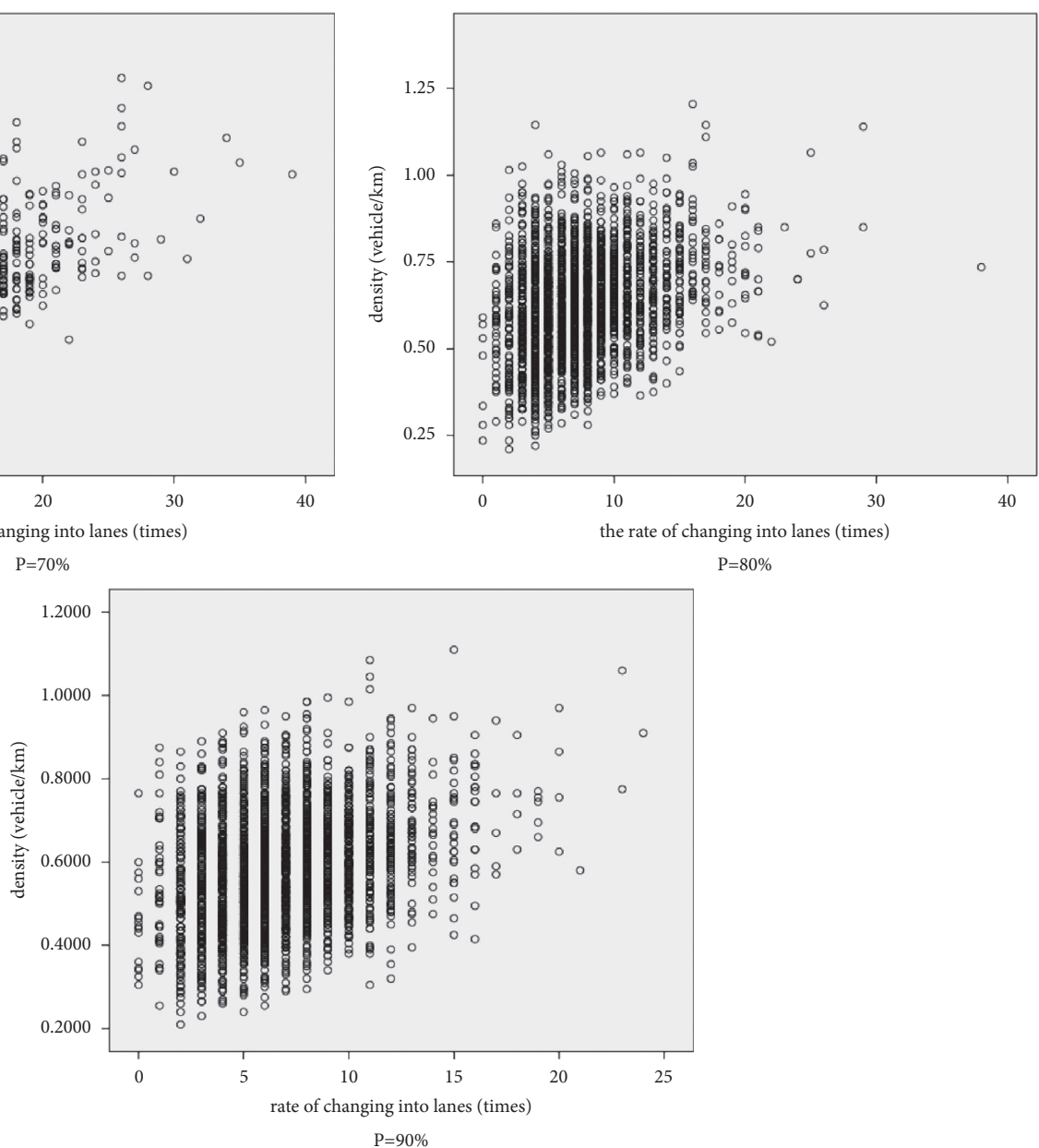

(a)

Figure 11: Continued. 

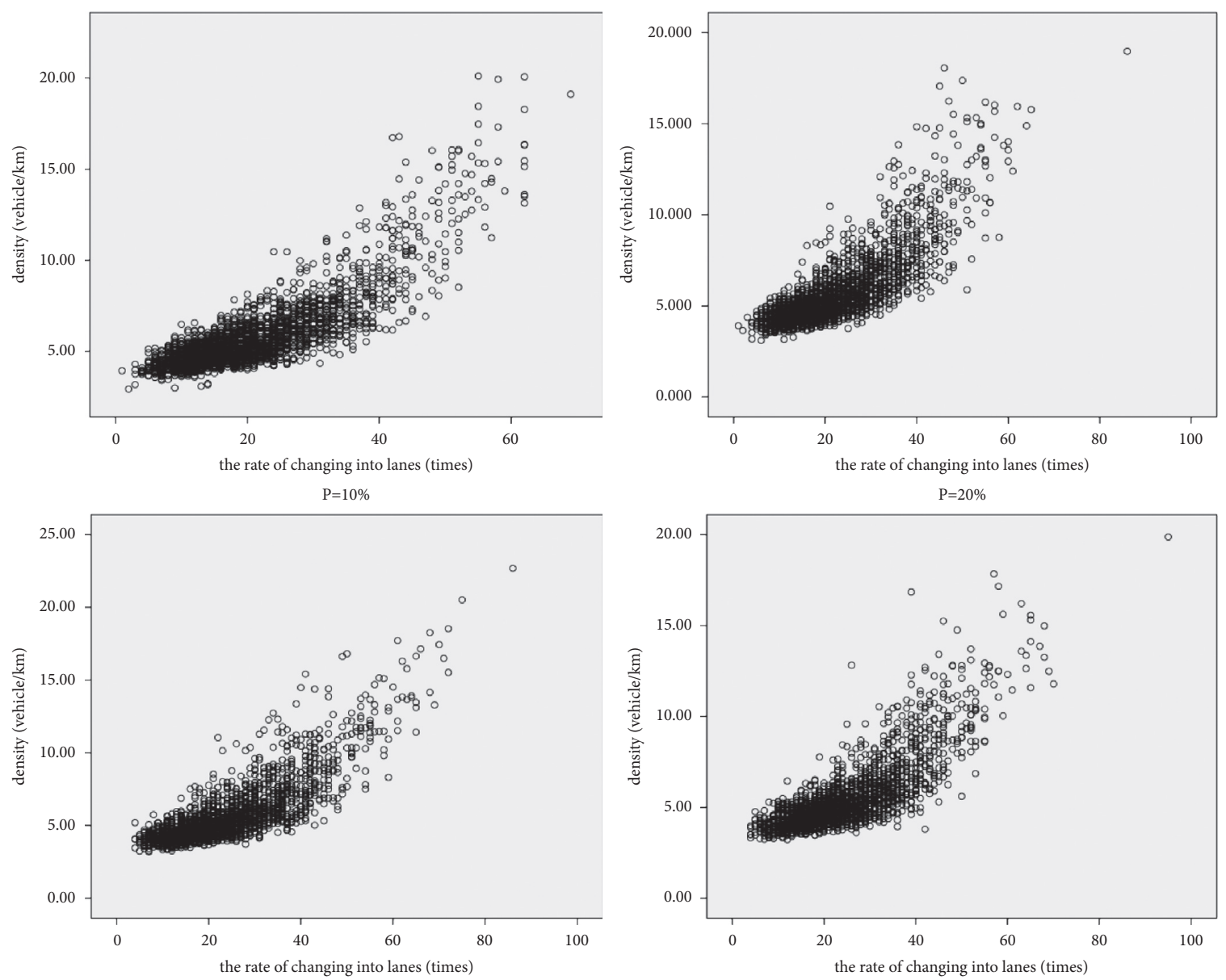

$\mathrm{P}=30 \%$
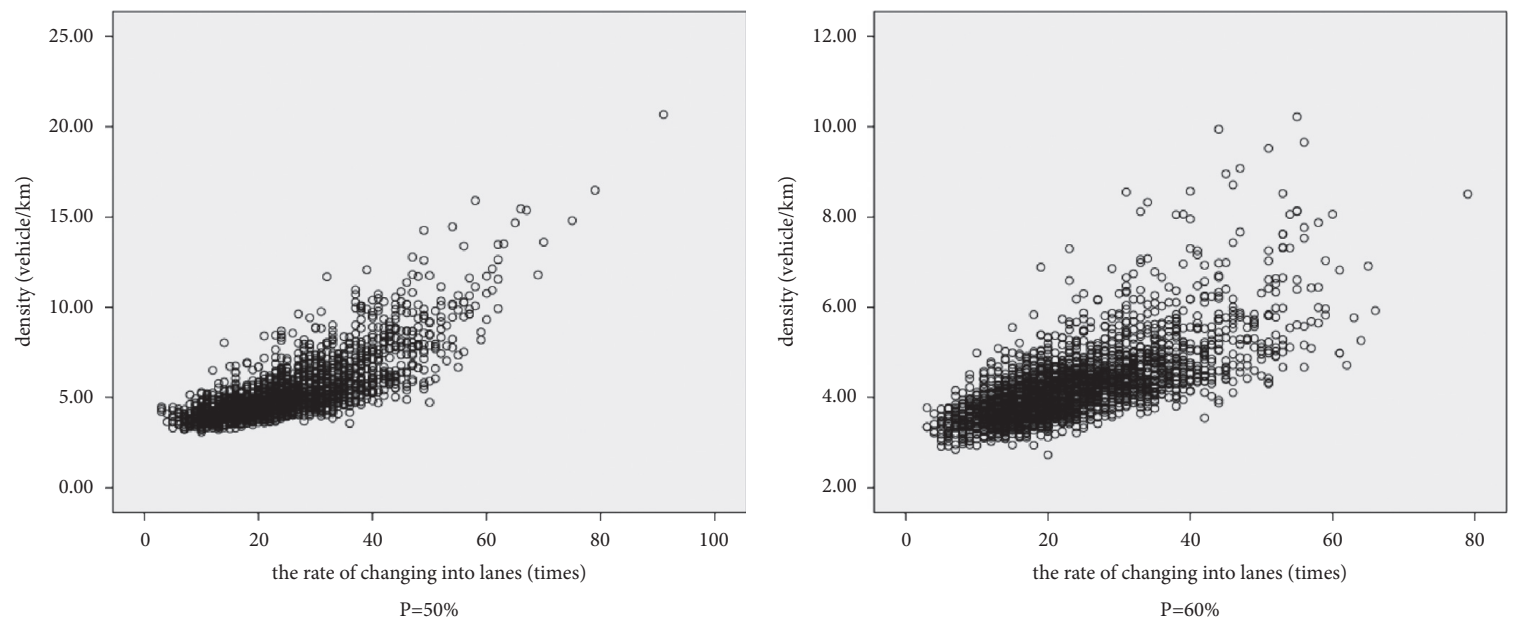

FIGURE 11(b): Continued. 

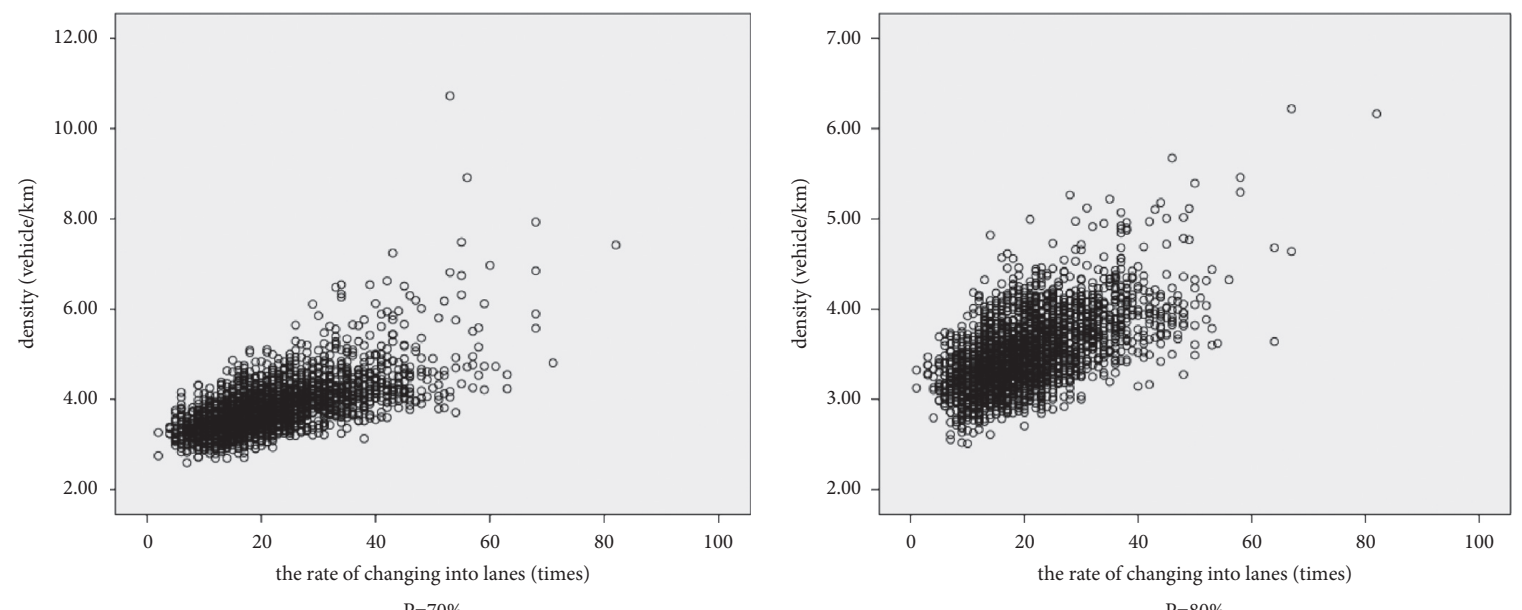

$\mathrm{P}=70 \%$ $\mathrm{P}=80 \%$

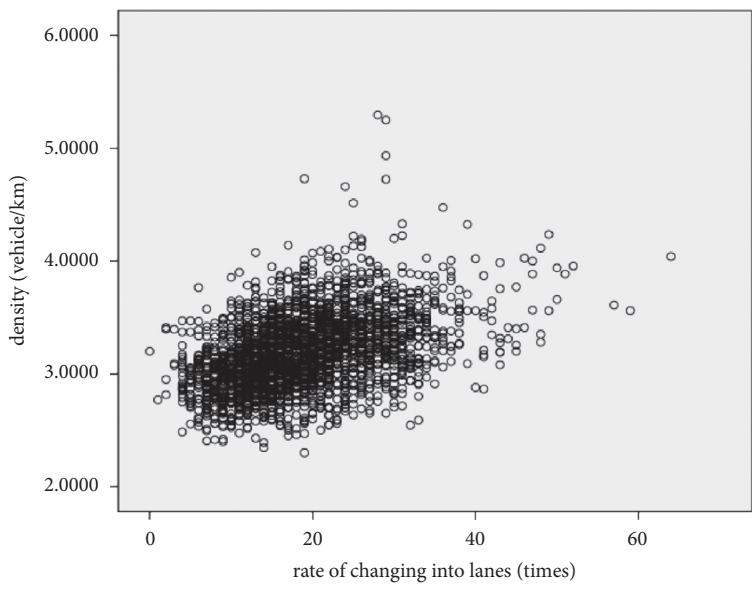

$\mathrm{P}=90 \%$

(b)

Figure 11: Continued. 

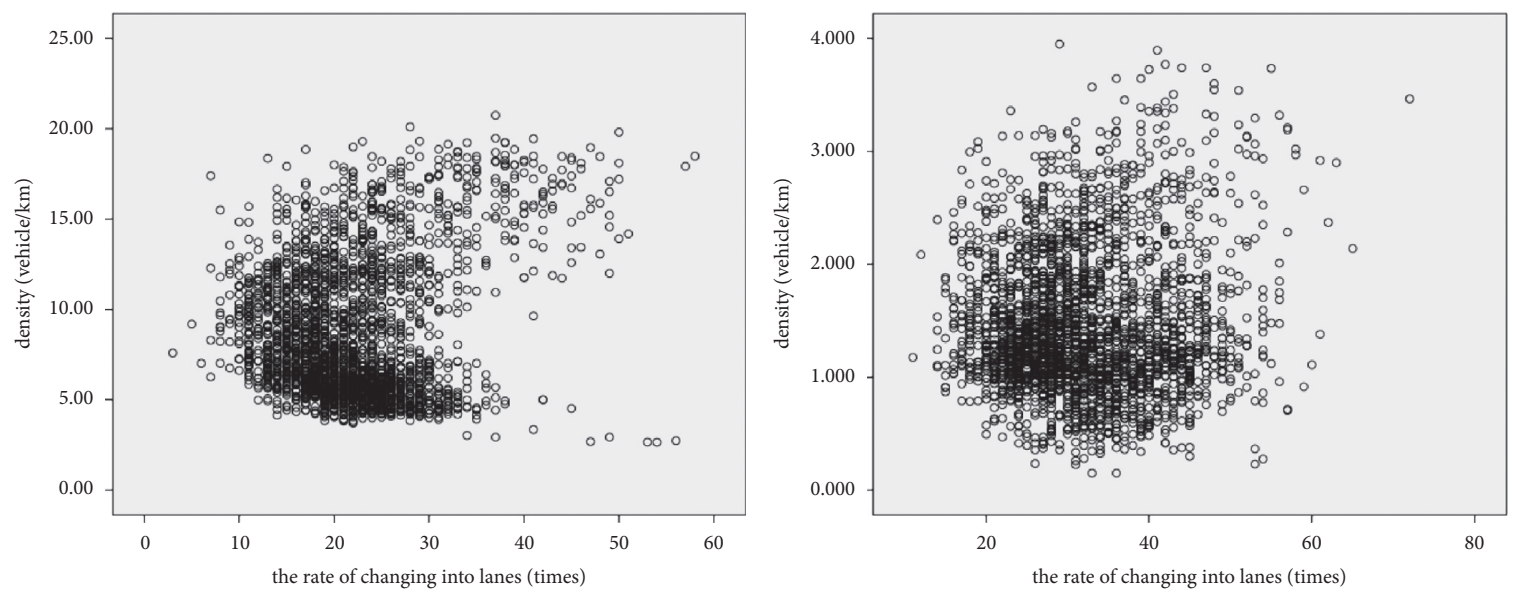

$\mathrm{P}=10 \%$
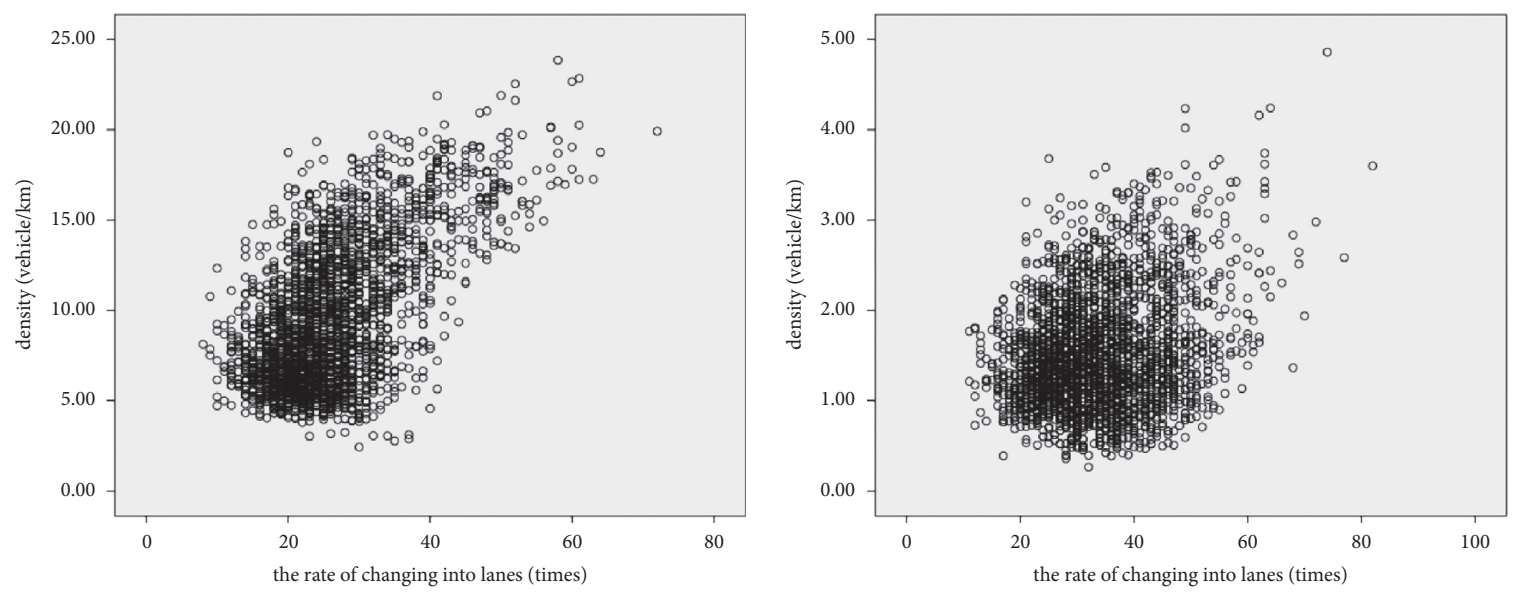

$\mathrm{P}=30 \%$
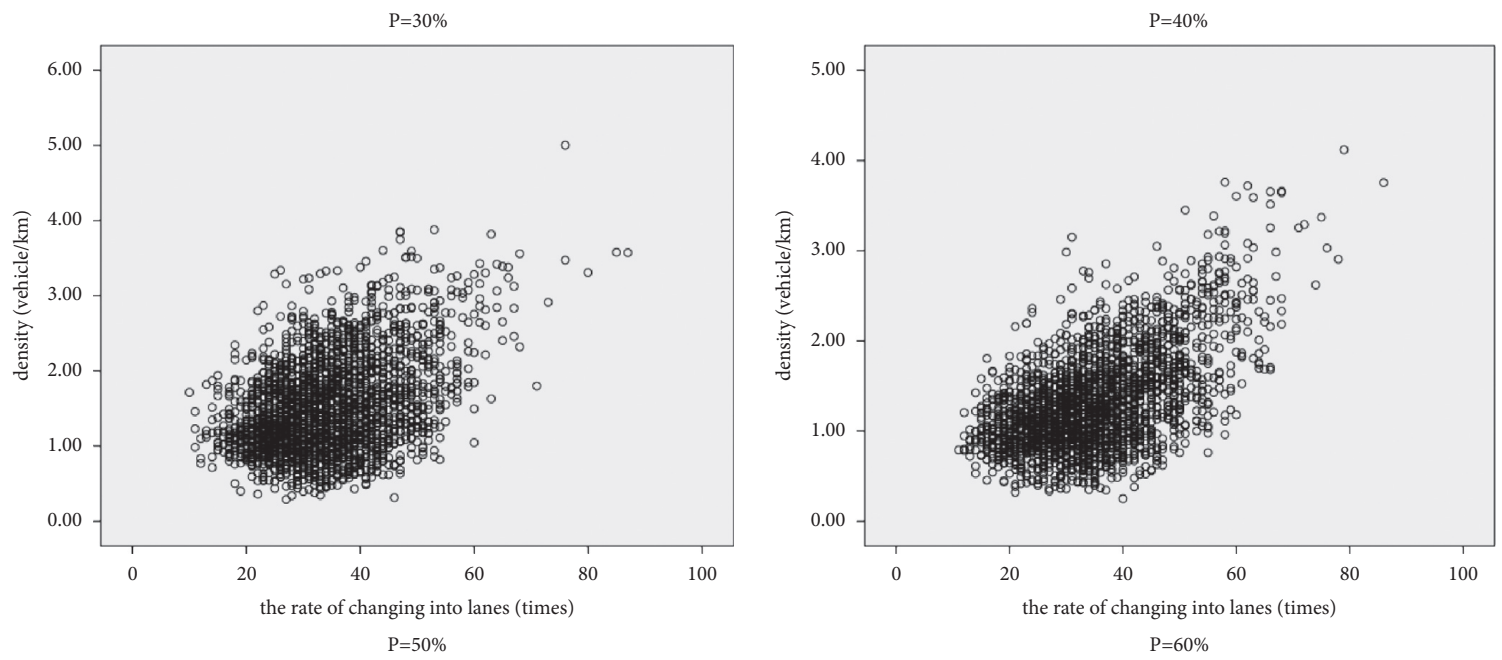

Figure $11(\mathrm{c})$ : Continued. 

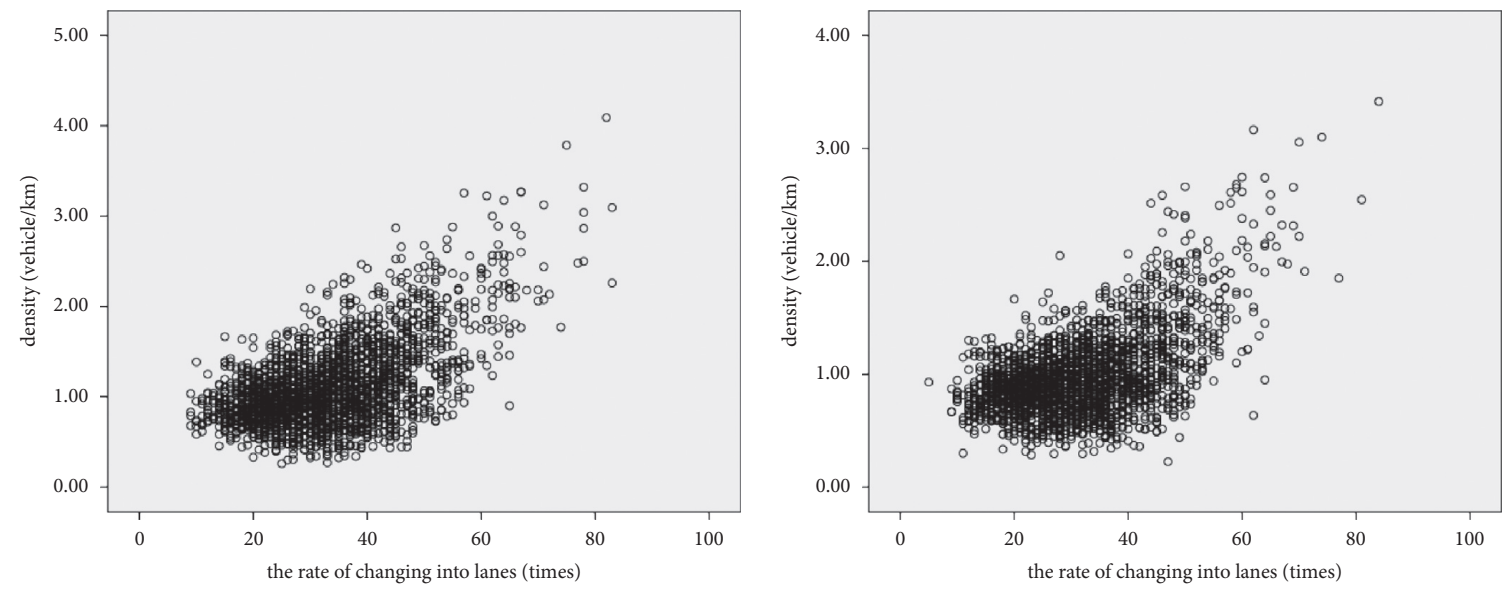

$\mathrm{P}=80 \%$

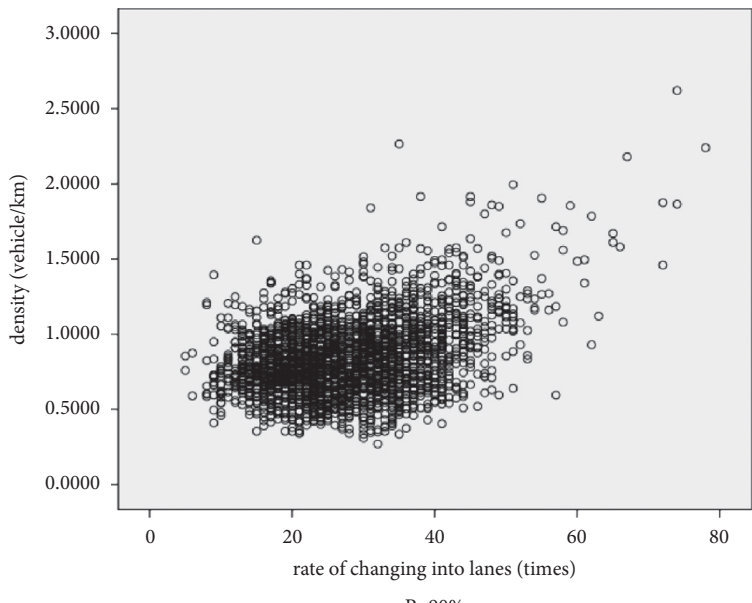

(c)

Figure 11: Continued. 

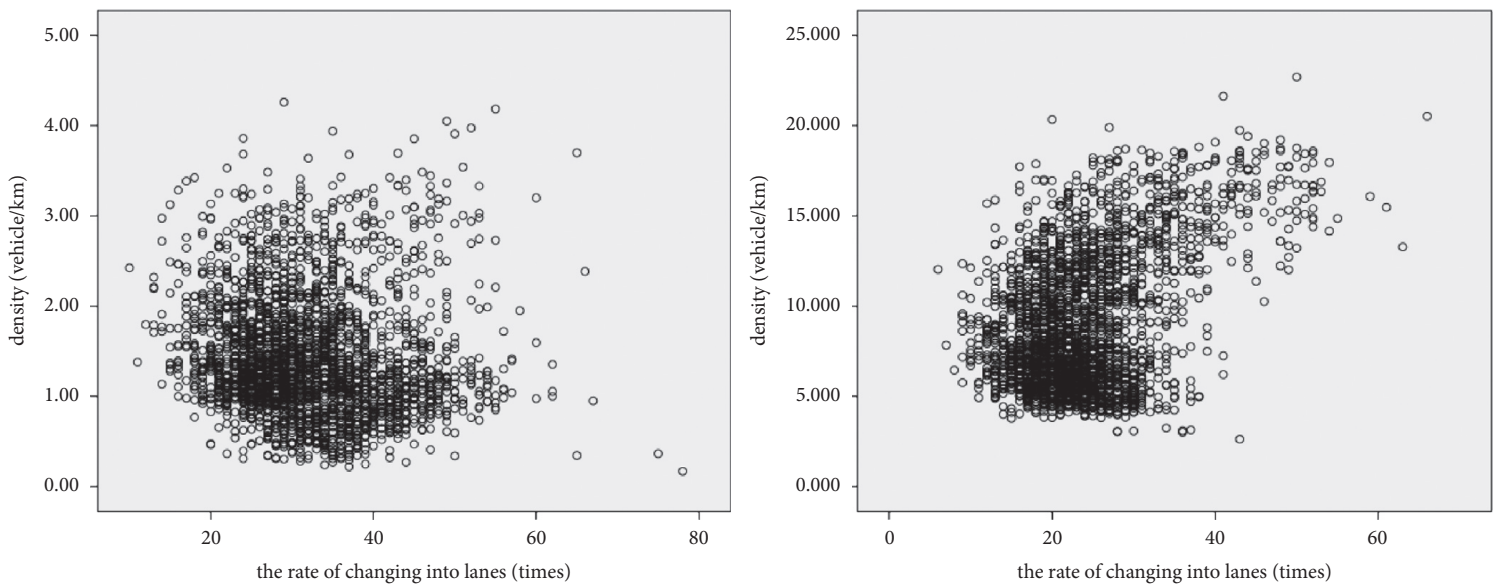

$\mathrm{P}=10 \%$
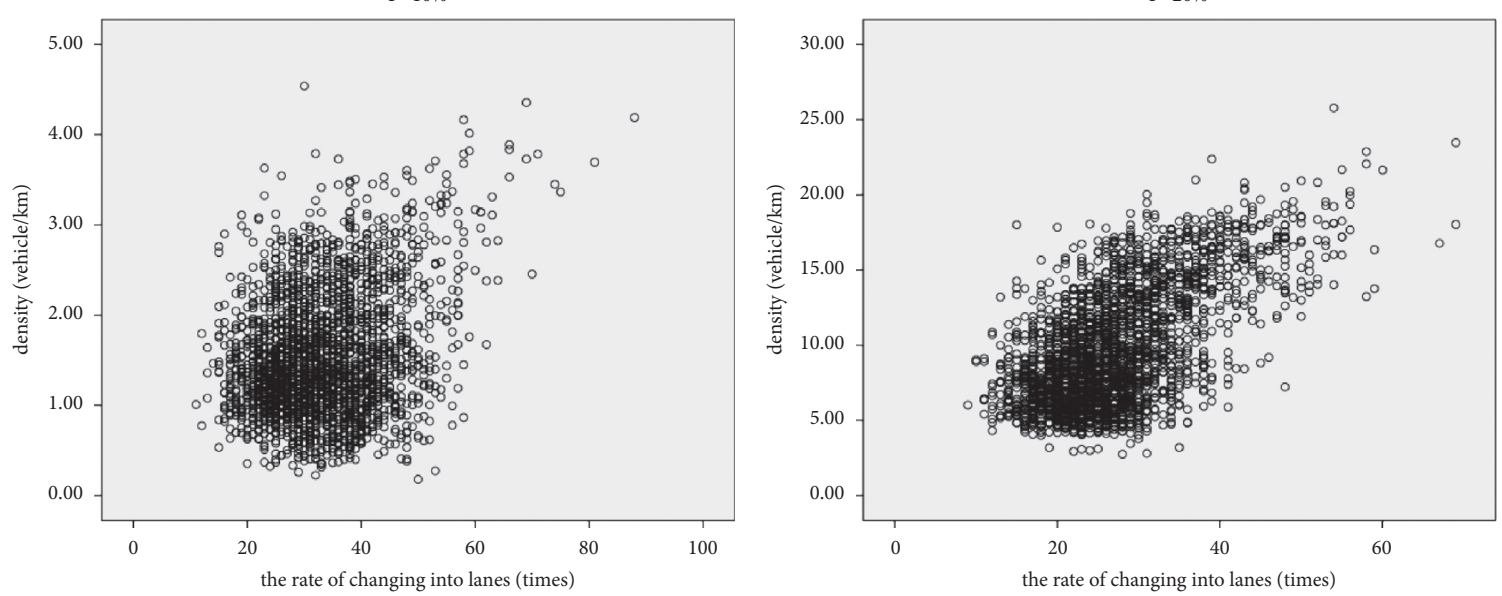

$\mathrm{P}=30 \%$

$\mathrm{P}=40 \%$
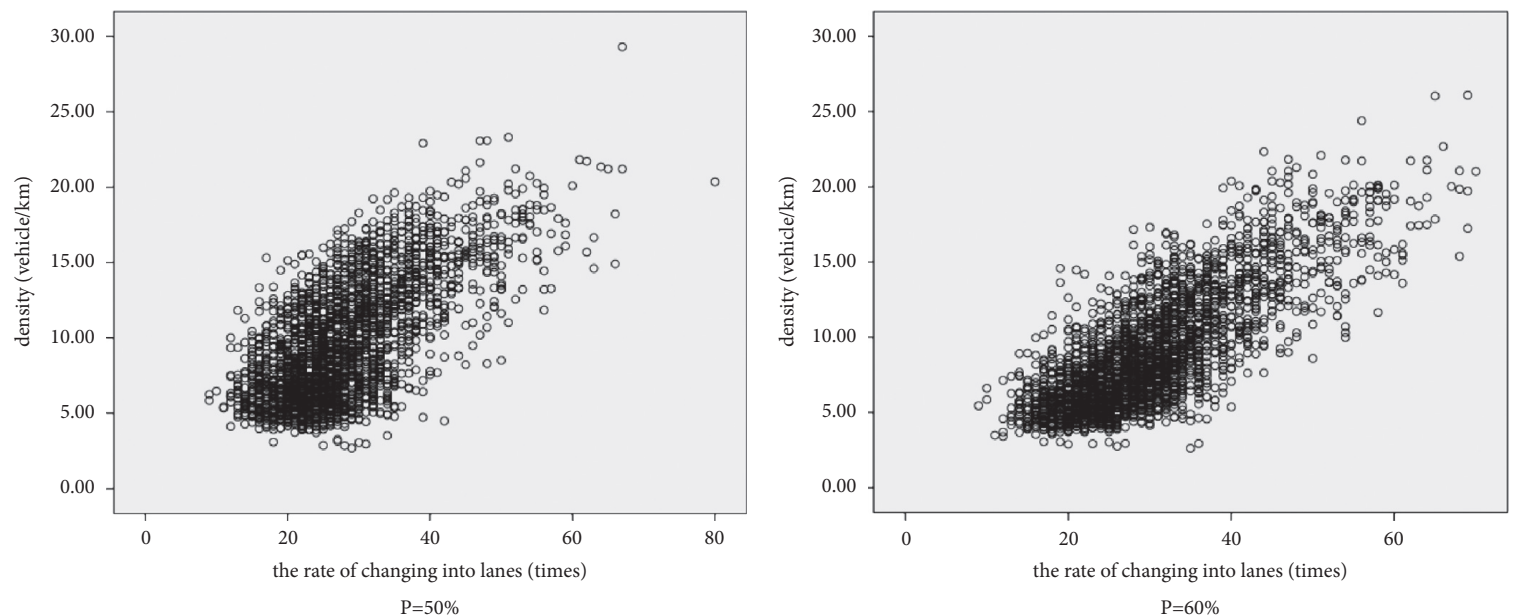

Figure 11(d): Continued. 

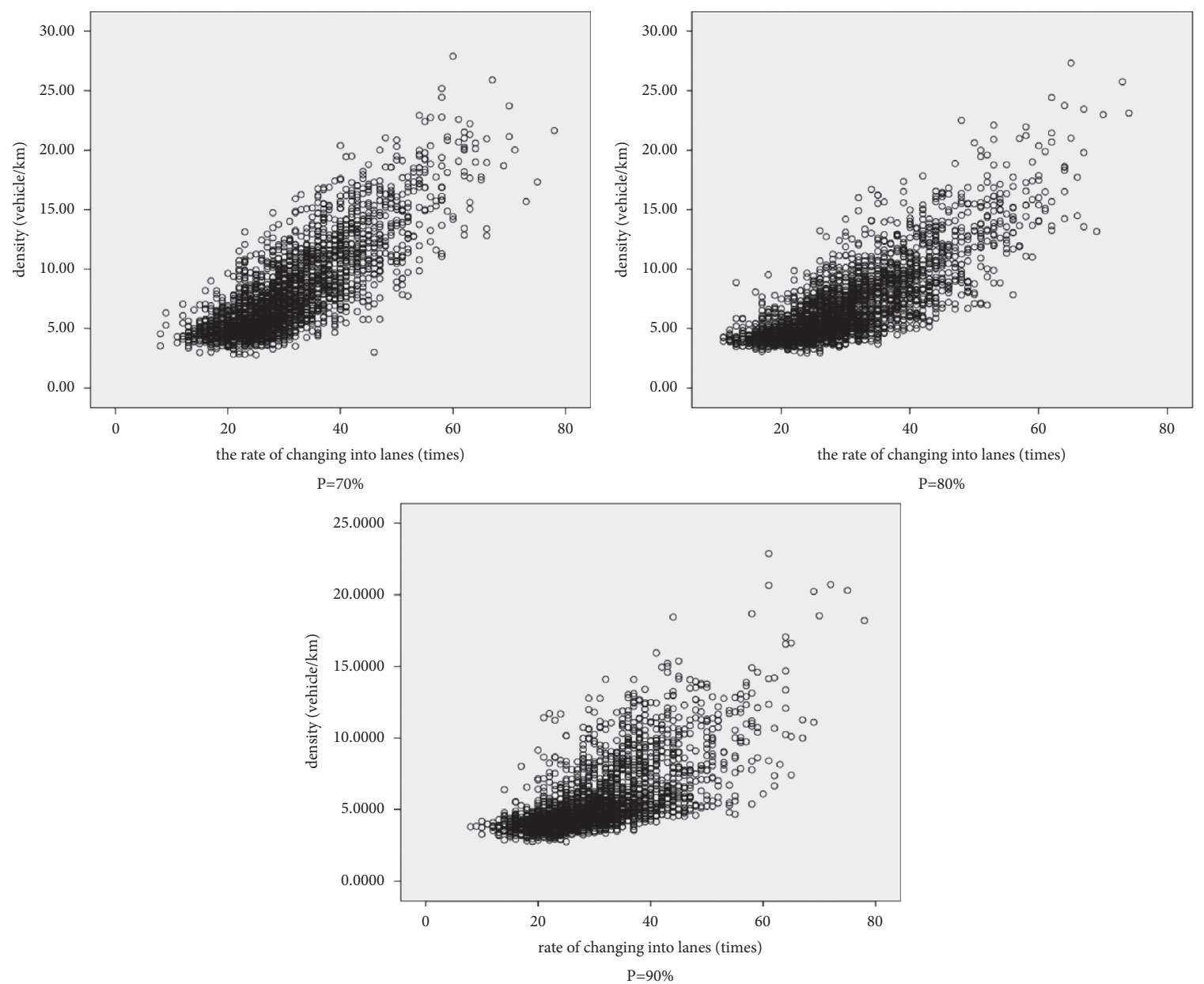

$\mathrm{P}=80 \%$

(d)

Figure 11: The relationship between density and the rate of changing into lanes by scatter plot. (a) The scatter plot of Lane 1. (b) The scatter plot of Lane 2. (c) The scatter plot of Lane 3. (d) The scatter plot of Lane 4.

relationship. Lane 1 has an impact on the relationship between traffic flow density and lane-changing behavior when it first with autonomous vehicles, and the linear relationship has gradually emerged. However, as the penetration rate of autonomous vehicles exceeds $50 \%$, most autonomous vehicles form queues. Vehicles that need to change lanes also follow a certain order, so they do not necessarily drive in Lane 1, which effectively reduces lane-changing behavior, so the relationship between density and lane-changing behavior gradually becomes discrete. This can also be seen in Figures 10 and 11. But in general, the relationship between the density and the behavior of changing lanes has developed towards a linear relationship with the penetration of autonomous vehicles. In particular, the degree of fit of Lane 2 is higher, as shown in Figures 10 and 11. It can also be clearly observed. Therefore, multiple regression can be used to describe the relationship between density and lane-changing behavior. Table 9 shows the parameter value interval under different permeability.

It can also be seen from Table 9 that under the same lane, the value interval of the same parameter under different permeability is not big. It further proves that the relationship between density and lane-changing behavior can be described by multiple regression. That is, with the continuous penetration of autonomous vehicles, there is an obvious multiple linear relationship between the density and the lane change rate. This relationship model can be used to describe the density and lane change in mixed traffic.

\section{Summary}

In this paper, empirical, simulation, and data-driven methods are used to study the relationship between singlelane traffic density and lane-changing behavior in mixed traffic on a typical 4-lane ring-shaped urban expressway under different autonomous vehicle penetration rates. Through empirical research, the traffic density, the rate of changing into lanes, and the rate of changing out lanes data of each lane of a ring-shaped urban expressway are obtained, and the usability of the data is verified through a data-driven method. Based on empirical research, a corresponding simulation model was established. Through simulation and 
TABLE 8: The relationship between the speed of a single lane and the lane change rate under different permeability by different fitting degrees.

\begin{tabular}{lccccccccc}
\hline & & $P=10 \%$ & $P=20 \%$ & $P=30 \%$ & $P=40 \%$ & $P=50 \%$ & $P=60 \%$ & $P=70 \%$ & $P=80 \%$ \\
\hline \multirow{4}{*}{ Lane 1 } & Multiple R & 0.569 & 0.571 & 0.647 & 0.607 & 0.602 & 0.484 & 0.423 & 0.399 \\
& R square & 0.324 & 0.326 & 0.418 & 0.368 & 0.362 & 0.234 & 0.179 & 0.159 \\
& Adjusted R square & 0.323 & 0.325 & 0.418 & 0.368 & 0.362 & 0.233 & 0.179 & 0.159 \\
& Standard error & 0.287 & 0.266 & 0.264 & 0.239 & 0.229 & 0.174 & 0.149 & 0.134 \\
\hline \multirow{2}{*}{ Lane 2 } & Multiple R & 0.850 & 0.828 & 0.845 & 0.837 & 0.824 & 0.740 & 0.675 & 0.606 \\
& R square & 0.722 & 0.685 & 0.715 & 0.700 & 0.679 & 0.547 & 0.456 & 0.367 \\
& Adjusted R square & 0.722 & 0.685 & 0.714 & 0.700 & 0.678 & 0.547 & 0.455 & 0.367 \\
& Standard error & 1.109 & 1.091 & 1.099 & 1.008 & 0.932 & 0.561 & 0.439 & 0.327 \\
\hline \multirow{4}{*}{ Lane 3 } & Multiple R & 0.636 & 0.627 & 0.648 & 0.630 & 0.668 & 0.716 & 0.697 & 0.674 \\
& R square & 0.404 & 0.393 & 0.420 & 0.397 & 0.446 & 0.513 & 0.485 & 0.455 \\
& Adjusted R square & 0.404 & 0.393 & 0.420 & 0.396 & 0.446 & 0.513 & 0.485 & 0.454 \\
& Standard error & 0.519 & 0.499 & 0.512 & 0.490 & 0.466 & 0.378 & 0.323 & 0.269 \\
\hline \multirow{2}{*}{ Lane 4} & Multiple R & 0.219 & 0.468 & 0.629 & 0.657 & 0.730 & 0.821 & 0.834 & 0.822 \\
& R square & 0.048 & 0.219 & 0.395 & 0.432 & 0.533 & 0.674 & 0.695 & 0.675 \\
& Adjusted R square & 0.047 & 0.219 & 0.395 & 0.432 & 0.533 & 0.673 & 0.695 & 0.675 \\
& Standard error & 3.731 & 3.354 & 3.055 & 2.941 & 2.696 & 2.266 & 2.119 & 1.929 \\
\hline
\end{tabular}

TABle 9: Regression formula parameter value interval table under different autonomous vehicle penetration rates.

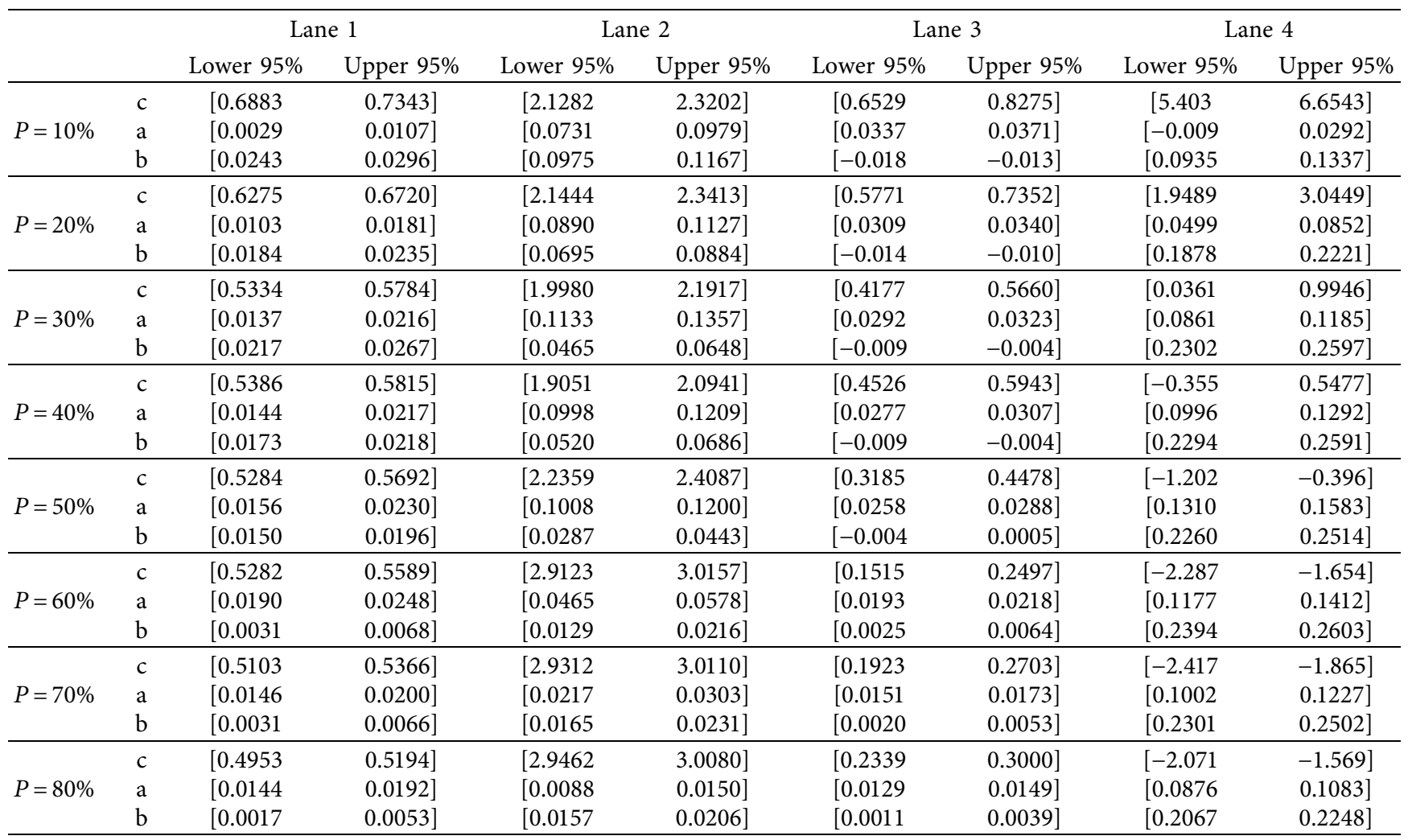

empirical data comparison, the relationship between density and the rate of changing into lanes, and the relationship between density and the rate of changing out lanes was found that under two methods the graphics are close. So, it is considered that the simulation model is close to the actual road conditions, and the density and lane-changing behavior under the penetration rate of autonomous vehicles can be studied. Based on the simulation model, simulation experiments were carried out when the penetration rate of autonomous vehicles was $10 \%, 20 \%, 30 \%, 40 \%, 50 \%, 60 \%$,
$70 \%$, and $80 \%$. The experiment obtained data on density, the rate of changing into lanes, and the rate of changing out lanes. Research using a data-driven approach found the following:

(1) The density of a single lane decreases to a certain extent with the penetration of autonomous vehicles. The downward trend of the two lanes farther from the entrance and exit is more obvious. Lane 3 and Lane 4, which are closer to the entrance and exit, first 
increase and then decrease. Although it reaches $80 \%$, the situation compared with no autonomous vehicle is still reduced, but there has been an upward trend in the front. The phenomenon indicates indicating that the penetration of autonomous vehicles has caused vehicles that can temporarily not drive in Lane 3 and Lane 4 to appear in those lanes. After the permeability reaches $50 \%$, the density decreases.

(2) The rate of changing into lanes and the rate of changing out lanes are closer with the addition of autonomous vehicles. When the penetration rate of autonomous vehicles is $10 \%$, all lanes show a significant decrease in lane-changing behavior. Then, with the penetration of autonomous vehicles, Lane 1 to Lane 2, the rate of changing into lanes and the rate of changing out of lanes has a relatively obvious downward trend, while Lane 3 and Lane 4 have no obvious trend. Especially, in Lane 3, as the penetration rate of autonomous vehicles increases, lanechanging behavior has increased. Lane 3 is still the main place where the behavior of changing in and out of lanes occurs.

(3) With the penetration of autonomous vehicles, the density, the rate of changing into lanes, and the rate of changing out lanes all tend to converge towards the mode.

(4) With the penetration of autonomous vehicles, the linear relationship between density and the rate of changing into lanes and the rate of changing out lanes appears. Although the data distribution of the three is still nonnormal, the degree of fit of the relationship obtained after multiple regression analysis is mostly above 0.6 under the conditions of different permeability and different lanes. It shows that the penetration of autonomous vehicles leads to a multiple linear relationship between density and lane-changing behavior.

In summary, we believe that autonomous vehicles have a certain effect on improving traffic on multilane circular urban expressways with large traffic volumes. When the penetration rate of autonomous vehicles exceeds $10 \%$, the density, the rate of changing into lanes, and the rate of changing out lanes will decrease to a certain extent. Although there is a slight upward trend with the increase in penetration rate, it will be lower for most lanes. In other words, after the permeability exceeds $50 \%$, the three parameters all show a downward trend. Finally, when the permeability reaches $80 \%$, compared with the case of no autonomous vehicle, the three parameters all have a certain degree of decline. At the same time, the relationship between density and the rate of changing into lanes and the rate of changing out lanes shows a certain degree of linearity with the penetration of autonomous vehicles, and the relationship can be described by a multiple regression model. The parameter value interval given by the model also has little difference, which can prove the stability of the model.
In addition, this paper does not test the applicability of the density-lane change rate model in mixed traffic on other roads. This will be the direction of the next research.

\section{Data Availability}

The data used in this research can be obtained from the corresponding author upon request.

\section{Conflicts of Interest}

The authors declare that there are no conflicts of interest regarding the publication of this study.

\section{Acknowledgments}

This research was supported by Applied Basic Research Programs and Technology Commission Foundation of Sichuan Province of China (Grand nos. 2019JDR0093, 2020JDR0253, 2021YJ0066, 2017JY0246, and 2017JY0269), Chengdu Science and Technology Project (Grand nos. 2017RK0000306ZF and 2017RK0000357ZF), and Key Scientific Research Fund of Xihua University (Grand no. Z17131). The authors would like to express their special thanks of gratitude to their colleagues for their support and help in the process of research and writing.

\section{References}

[1] A. Talebpour and H. S. Mahmassani, "Influence of connected and autonomous vehicles on traffic flow stability and throughput," Transportation Research Part C: Emerging Technologies, vol. 71, pp. 143-163, 2016.

[2] Y.-Y. Qin, H. Wang, W. Wang, and W. Qian, "Stability analysis and fundamental diagram of heterogeneous traffic flow mixed with cooperative adaptive cruise control vehicles," Acta Physica Sinica, vol. 66, no. 9, Article ID 094502, 2017.

[3] X. Chang, H. J. Li, J. Rong, Q. Lingqiao, and Y. Yanfang, "Analysis on fundamental diagram model for mixed traffic flow with connected vehicle platoons," Journal of Southeast University (Natural Science Edition), vol. 50, no. 4, pp. 782788, 2020.

[4] L. Gen and C. Juan, "Exploring the effects of traffic density on merging behavior," IEEE Access, vol. 7, pp. 51608-51619, 2019.

[5] L. Huang, H. Guo, R. Zhang, H. Wang, and J. Wu, "Capturing drivers' lane changing behaviors on operational level by data driven methods," IEEE Access, vol. 6, pp. 57497-57506, 2018.

[6] C. Wang, Q. Sun, Z. Li, H. Zhang, and K. Ruan, "Cognitive competence improvement for autonomous vehicles: a lane change identification model for distant preceding vehicles," IEEE Access, vol. 7, pp. 83229-83242, 2019.

[7] Y. Dou, Y. Fang, C. Hu, R. Zheng, and F. Yan, "Gated branch neural network for mandatory lane changing suggestion at the on-ramps of highway," IET Intelligent Transport Systems, vol. 13, no. 1, pp. 48-54, 2019.

[8] L. Gen, F. Song, M. Jianxiao, and C. Juan, "Modeling merging acceleration and deceleration behavior based on gradient boosting decision tree," JournalofTransportation Engineering ,Part A:Systems, vol. 146, no. 7, Article ID 05020005, 2020.

[9] Ministry of Housing and Urban-Rural, Development of the People's Republic of China.Design Discipline of Urban Expressway[S], China Architecture \& Building Press, Beijing, China, 2009. 
[10] W.-X. Zhu and H. M. Zhang, "Analysis of mixed traffic flow with human-driving and autonomous cars based on carfollowing model," Physica A: Statistical Mechanics and Its Applications, vol. 496, pp. 274-285, 2018.

[11] L. Gen, M. Jianxiao, and Y. Zhen, "Characteristics of heavy vehicle discretionary lane change based on trajectory data," Transportation Research Record, Article ID 03611981211051337, 2021.

[12] Z. Yao, H. Jiang, and Y. Cheng, "Integrated schedule and trajectory optimization for connected automated vehicles in a conflict zone," IEEE Transactions on Intelligent Transportation Systems, pp. 1-11, 2020.

[13] X. Wang, W. Li, C. Yin, S. Zeng, and P. Liu, "A multipleparameter approach for short-term traffic flow prediction," Modern Physics Letters B, vol. 35, no. 14, Article ID 2150245, 2021.

[14] W. Li, S. Chen, X. Wang, and Z. Huang, "A hybrid approach for short-term traffic flow forecasting based on similarity identification," Modern Physics Letters B, vol. 35, no. 13, Article ID 2150212, 2021.

[15] C. L. Davis, "Effect of adaptive cruise control systems on traffic flow," Physical Review E - Statistical, Nonlinear and Soft Matter Physics, vol. 69, no. 6, Article ID 066110, 2004.

[16] M. W. Levin and S. D. Boyles, "A multiclass cell transmission model for shared human and autonomous vehicle roads," Transportation Research Part C: Emerging Technologies, vol. 62, pp. 103-116, 2016.

[17] D. Xie, X. Zhao, and Z. He, "Heterogeneous traffic mixing regular and connected vehicles: modeling and stabilization," IEEE Transactions on Intelligent Transportation Systems, vol. 20, no. 6, pp. 2060-2071, 2019.

[18] S. Wang, J. Zhao, C. Shao, and C. Yin, "Truck traffic flow prediction based on LSTM and GRU methods with sampled GPS data," IEEE Access, vol. 8, pp. 208158-208169, 2020.

[19] G. G. Newell, "A moving bottleneck," Transportation Research Part B, vol. 32, no. 8, pp. 531-537, 1998.

[20] J. A. Laval and C. F. Daganzo, "A hybrid model of traffic flow: impacts of roadway geometry on capacity," in Proceedings of the TRB 2003 Annual Meeting CD-ROM 2003, North Avenue, Atlanta, GA, USA, 2003.

[21] J. A. Laval and C. F. Daganzo, "Lane-changing in traffic streams," Transportation Research Part B, vol. 40, pp. 251-264, 2006.

[22] W.-L. Jin, "Kinematic wave-theory of lane-changing traffic flow," Transportation Research Part B, vol. 44, no. 8-9, pp. 1001-1021, 2009.

[23] Transportation Research Board, Highway Capacity Manual, HCM2010), USA, 2010.

[24] K. Liu, J. W. Gong, K. Arda, H. Y. Chen, and O. Umit, "Dynamic modeling and control of high-speed automated vehicles for lane change maneuver," IEEE Transactions on Intelligent Vehicles, vol. 3, no. 3, pp. 329-339, 2018.

[25] Y. Xing, C. Lv, H. J. Wang et al., "Driver lane change intention inference for intelligent vehicles: framework, survey, and challenges," IEEE Transactions on Vehicular Technology, vol. 68, no. 5, pp. 4377-4390, 2019.

[26] X. P. Gu, Y. P. Han, and J. F. Yu, "A novel lane-changing decision model for autonomous vehicles based on deep autoencoder network and XGBoost," IEEE Access, vol. 8, pp. 9846-9863, 2020.

[27] L. Gen, P. Yiyong, Y. Zhen, and M. Jianxiao, "Modelling vehicle merging position selection behaviors based on a finite mixture of linear regression models," IEEE Access, vol. 7, 2019.
[28] N. Ding and X. H. Meng, W. G. Xia, D. Wu, L. Xu, and B. C. Chen, Multi-vehicle coordinated lane change strategy in the roundabout under Internet of vehicles based on game theory and cognitive computing," IEEE Transactions on Industrial Informatics, vol. 14, no. 8, pp. 1-8, 2015.

[29] B. Li, Y. M. Zhang, Y. H. Feng, Y. Zhang, Y. M. Ge, and Z. J. Shao, "Balancing computation speed and quality: a decentralized motion planning method for cooperative lane changes of connected and automated vehicles," IEEE Transactions on Intelligent Vehicles, vol. 3, no. 3, pp. 340-350, 2018.

[30] T. T. Li, J. P. Wu, C. Y. Chan et al., "A cooperative lane change model for connected and automated vehicles," IEEE Access, vol. 8, pp. 1-7, 2019.

[31] Y. Zheng, B. Ran, X. Qu, J. Zhang, and Y. Lin, "Cooperative lane changing strategies to improve traffic operation and safety nearby freeway off-ramps in a connected and automated vehicles environment," IEEE Transactions on Intelligent Transportation Systems, pp. 1-10, 2020.

[32] X. Liu, J. Liang, and B. Xu, "A deep learning method for lane changing situation assessment and decision making," IEEE Access, vol. 7, pp. 133749-133759, 2019.

[33] Q. S. Zhang and Y. P. Zhang, Analysis of Road Traffic Capacity, China Communication Press, Beijing, China, 2002.

[34] J. Li, Traffic Engineer, China Communication Press, Beijing, China, 2007. 